\title{
CONVERGENCE AND STOCHASTIC HOMOGENIZATION OF A CLASS OF NONLINEAR TIME DELAYS REACTION-DIFFUSION EQUATIONS
}

\author{
OMAR ANZA HAFSA, JEAN PHILIPPE MANDALLENA, AND GÉRARD MICHAILLE
}

\begin{abstract}
Stability under a variational convergence of nonlinear time delays reaction-diffusion equations is discussed. Problems considered cover various models of population dynamics or diseases in heterogeneous environments where delays terms may depend on the space variable. As a consequence a stochastic homogenization theorem is established and applied to vector disease and logistic models. The results illustrate the interplay between the growth rates and the time delays which are mixed in the homogenized model.
\end{abstract}

\section{Contents}

1. Introduction 2

Notation 3

\begin{tabular}{lll}
\hline 2. & The time-delays operator & 3
\end{tabular}

$\begin{array}{lll}2.1 . & \text { Integration with respect to vector measures } & 3\end{array}$

2.2. Time delays-operator associated with vector measures 4

2.3. Examples of time-delays operators 5

3. Reaction diffusion problems associated with convex functionals of the calculus of variations $\begin{array}{ll}\text { and DCP-structured reaction functionals } & 8\end{array}$

3.1. The class of DCP-structured reaction functionals 9

3.2. Some examples of DCP-structured reaction functions coming from ecology and biology models

3.3. Existence and uniqueness of bounded nonnegative solution 12

$\begin{array}{lll}\text { 4. General convergence theorems for a class of delays nonlinear reaction-diffusion problems } & 17\end{array}$

$\begin{array}{lll}\text { 4.1. } & \text { Stability at the limit } & 18\end{array}$

\begin{tabular}{|ll}
4.2. & Non stability at the limit for the reaction functional: convergence with mixing effect \\
between growth rates and time delays at the limit & 24
\end{tabular}

5. Stochastic homogenization of distributed delays reaction diffusion problems 25

$\begin{array}{lll}\text { 5.1. The random diffusion part } & 25\end{array}$

$\begin{array}{lll}\text { 5.2. } & \text { The random reaction part } & 26\end{array}$

5.3. Almost sure convergence to the homogenized reaction-diffusion problem 28

\begin{tabular}{|l|l}
\hline Appendix A. Vector measures & 31
\end{tabular}

\begin{tabular}{|lll}
\hline Appendix B. Notion of CP-structured reaction functionals & 32
\end{tabular}

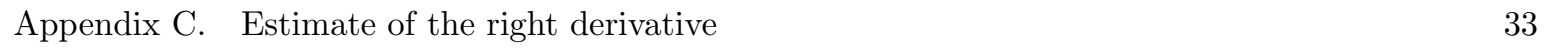

Appendix D. An alternative proof of Theorem 4.1 |in the case of a single time delay 34

\begin{tabular}{|ll|l|l|l|}
\hline Appendix E. Appplication of Theorem & 5.1 & to some examples & 36
\end{tabular}

E.1. Homogenization of vector disease models 36

E.2. Homogenization of delays logistic equations with immigration 39

$\begin{array}{lr}\text { References } & 40\end{array}$

(Omar Anza Hafsa, Jean Philippe Mandallena, Gérard Michaille) UNIVERSite DE NIMES, Laboratoire MIPA, Site des Carmes, Place Gabriel Péri, 30021 Nîmes, France.

E-mail addresses: (Omar Anza Hafsa, Jean Philippe Mandallena, Gérard Michaille) omar.anza-hafsa@unimes.fr, jean-philippe.mandallena@unimes.fr, gerard.michaille@gmail.com.

2010 Mathematics Subject Classification. 35K57, 35B27, 49J45.

Key words and phrases. Convergence of time delays reaction-diffusion equations, Mosco-convergence, stochastic homogenization, vector disease and logistic models. 


\section{INTRODUCTION}

In this paper we continue to investigate further the convergence of reaction-diffusion problems discussed in 2, extending it to the case when the reaction term includes single or infinite time delays which may depend on the space variable. More precisely, we are concerned with the convergence and the stochastic homogenization of sequences $\left(\mathcal{P}_{n}\right)_{n \in \mathbb{N}}$ of reaction-diffusion equations of the type

$$
\left(\mathcal{P}_{n}\right)\left\{\begin{array}{l}
\frac{d u_{n}}{d t}(t)+\partial \Phi_{n}\left(u_{n}(t)\right) \ni F_{n}\left(t, u_{n}(t), v_{n}(t)\right) \text { for a.e. } t \in(0, T) \\
u_{n}(t)=\eta_{n}(t) \text { for all } t \in(-\infty, 0],
\end{array}\right.
$$

where the function $v_{n}$ is connected to the function $u_{n} \in L^{2}\left(0, T, L^{2}(\Omega)\right)$ via a family $\left(\mathbf{m}_{t}^{n}\right)_{t \geq 0}$ of $L^{\infty}(\Omega)$ valued Borel vector measures $\mathbf{m}_{t}^{n}$ (see Definition A.1) according to the formula

$$
v_{n}(t)=\int_{-\infty}^{t} u_{n}(s) d \mathbf{m}_{t}^{n}(s) .
$$

The function $\eta_{n}$, referred to as history function, is nonnegative, bounded, absolutely continuous in $C\left((-\infty, 0], L^{2}(\Omega)\right)$, and satisfies $\eta_{n}(0) \in \operatorname{dom}\left(\Phi_{n}\right)$. When $\mathbf{m}_{t}^{n}=\delta_{t-\tau}$, where $\tau>0$ does not depend on the space variable, we recover the single time delay reaction-diffusion problems

$$
\left(\mathcal{P}_{n}\right)\left\{\begin{array}{l}
\frac{d u_{n}}{d t}(t)+\partial \Phi_{n}\left(u_{n}(t)\right) \ni F_{n}\left(t, u_{n}(t), u_{n}(t-\tau)\right) \text { for a.e. } t \in(0, T) \\
u_{n}(t)=\eta_{n}(t) \text { for all } t \in[-\tau, 0] .
\end{array}\right.
$$

When $\mathbf{m}_{t}^{n}=\frac{1}{\#\left(\tau_{n}\left(\mathbb{R}^{N}\right)\right)} \delta_{t-\tau_{n}(\cdot)}$ where $\tau_{n}: \mathbb{R}^{N} \rightarrow[0,+\infty)$ is a Borel measurable function taking a finite number of values, $\left(\mathcal{P}_{n}\right)$ is a single delay times reaction diffusion problem whose delay times may vary with respect to the space variable. When $\mathbf{m}_{t}^{n}=\sum_{k \in \mathbb{N}} \mathbf{d}_{k}^{n} \delta_{t-\tau_{k}}$, with $\mathbf{d}_{k}^{n} \in L^{\infty}(\Omega)$, the reaction term of $\left(\mathcal{P}_{n}\right)$ includes a multiple time delays which depends of the space variable through the functions $\mathbf{d}_{k}^{n}$. When $\mathbf{m}_{t}^{n}=\mathcal{K}_{n}(\cdot, t-\tau) d \tau$, the reaction term of $\left(\mathcal{P}_{n}\right)$ is associated with diffuse delays, distributed according to a kernel $\mathcal{K}_{n}$ (see Examples 2.1, 2.2 and 2.3 for the notation and further details). For a general $L^{\infty}(\Omega)$-valued Borel vector measure $\mathbf{m}_{t}^{n},\left(\mathcal{P}_{n}\right)$ is associated with potentially, a mixture of diffuse and discrete time delays.

The diffusion term is the subdifferential of a convex integral functional $\Phi_{n}: L^{2}(\Omega) \rightarrow \mathbb{R} \cup\{+\infty\}$, whose domain contains the boundary conditions. For the reaction term, we introduce the notion of DCPstructured reaction functionals. Roughly, the reaction functional $F_{n}:[0,+\infty) \times L^{2}(\Omega) \times L^{2}(\Omega) \rightarrow \mathbb{R}^{\Omega}$ is such that for fixed $v \in L^{2}(\Omega),(t, u) \mapsto F_{n}(t, u, v)$ is a CP-structured reaction functional as defined in 2. From condition (DCP), each $\left(\mathcal{P}_{n}\right)$ satisfies a comparison principle with respect to the datas, hence admits a nonnegative and bounded solution. To be self contained we summarize in Appendix B the definition of CP-structured reaction functionals as well as the corresponding existence theorem to which we refer.

Problems $\left(\mathcal{P}_{n}\right)$ model various situations involving for example reaction time, maturation period, resource regeneration time, time required for substance production, mating processes, or incubation period in vector disease models; see Examples (3.1), (3.2), (3.3), and (3.4) with the appropriate references. Among the very large literature related to analysis of asymptotic behavior, boundedness, positiveness and equilibrium of solutions of delay differential equation with diffusion, refer to [13, 14, 15, 18, and references therein. For existence, comparison principle for semilinear reaction diffusions equations with infinite delay refer to 23,25 .

In Section 3 we prove existence and uniqueness of bounded nonnegative strong solutions of problems of the type $\left(\mathcal{P}_{n}\right)$ which admit a right derivative at each $t \in[0, T$. The proof is based on 2, Theorem 3.1] combined with a fixed point procedure. In Section 4 under the hypotheses of Mosco-convergence of functionals $\Phi_{n}$, weak convergence of the vector measure $\mathbf{m}_{t}^{n}$ for each fixed $t$ in $[0, T]$, and a suitable convergence of $F_{n}$, we establish a stability result (Theorem 4.1), i.e., the convergence of $\left(\mathcal{P}_{n}\right)$ toward a distributed time delays reaction-diffusion problem $(\mathcal{P})$ of the same type. In a second theorem (Theorem 4.2 we establish the convergence toward a delay reaction-diffusion problem $(\mathcal{P})$ where a mixing effect appears in the limit reaction functional between the limit growth rates and the distributed time delays. In 
the case of single time delay, we also propose in Appendix $\mathrm{D}$ a constructive alternative proof, by applying suitably a finite number of times the convergence result established in $[2]$ to each $\left(\mathcal{P}_{n, i}\right), i=1 \ldots\left[\frac{T}{\tau}\right]+1$

$$
\left(\mathcal{P}_{n, i}\right)\left\{\begin{array}{l}
\frac{d u_{n}^{i}}{d t}(t)+\partial \Phi_{n}\left(u_{n}^{i}(t)\right) \ni F_{n}^{i}\left(t, u_{n}^{i}(t)\right) \text { for a.e. } t \in((i-1) \tau, i \tau) \\
u_{n}^{i}((i-1) \tau)=u_{n}^{i-1}((i-1) \tau)
\end{array}\right.
$$

where $F_{n}^{i}$ is the CP-structured functional defined by $F_{n}^{i}\left(t, u_{n}^{i}(t)\right):=F_{n}\left(t, u_{n}^{i}(t), u_{n}^{i-1}(t)\right)$, and $u_{n}^{0}:=\eta_{n}$ in $[-\tau, 0]$.

Stochastic homogenization is addressed in Section 5. The main result, Theorem 5.1, based on Theorems 4.2), states that a mixing phenomena appears in the homogenized reaction functional between the various growth rates and time delays. This phenomena occurs in the homogenization framework when the time-delays are strongly oscillating with respect to the space variable. The result is illustrated in Appendix E through the homogenization of a vector disease model and a delay logistic equation with immigration. In the first example, the growth rate of the uninfected population and the time delays coefficients of the homogenized problem are mixed in such a way that the effective multiple delays is associated with a measure incorporating the growth rate. In the second example, we can interpret the homogenized problem as a delay logistic diffusion equation modeling the evolution of a density population spreading in an homogeneous environment. The carrying capacity is now function of the growth rate and time dependent. For diffusion in random media and homogenization refer to 2, 8, 11, 10, 19, 20, for stochastic homogenization of Hamilton Jacobi equations refer to [3, 16, 24, and for stochastic homogenization of moving interfaces refer to [7, 9] and references therein.

Notation. We denote by $\mathcal{L}_{N}$ the Lebesgue measure in $\mathbb{R}^{N}$, by $\Omega$ a domain of $\mathbb{R}^{N}$ of class $C^{1}$ with outer unit normal $\mathbf{n}$, and by $\Gamma$ a subset of its boundary $\partial \Omega$ with positive $\mathcal{H}^{N-1}$-Hausdorf measure. To shorten the notation, we sometimes write $X$ to denote the Hilbert space $L^{2}(\Omega)$ equipped with its standard scalar product and its associated norm, denoted by $\langle\cdot, \cdot\rangle$ and $\|\cdot\|_{X}$ respectively. All along the paper we use the same notation $|\cdot|$ to denote the norms of the euclidean spaces $\mathbb{R}^{d}, d \geq 1$, and by $\xi \cdot \xi^{\prime}$ the standard scalar product of two elements $\xi, \xi^{\prime}$ in $\mathbb{R}^{d}$. We also denote by $\xi \odot \xi^{\prime}$ the Hadamard (or Schur) product of two elements $\xi$ and $\xi^{\prime}$ in $\mathbb{R}^{d}$. For any topological space $\mathbb{T}$, we denote by $\mathcal{B}(\mathbb{T})$ its Borel field, and by $C_{c}(\mathbb{T}, X)$ the space of continuous function from $\mathbb{T}$ into $X$, with compact support. For any sequence $\left(\Phi_{n}\right)_{n \in \mathbb{N}}$ of functionals $\Phi_{n}: X \rightarrow \mathbb{R} \cup\{+\infty\}$, we denote by $\Phi_{n} \stackrel{M}{\rightarrow} \Phi$ the Mosco-convergence of $\Phi_{n}$ to the functional $\Phi: X \rightarrow \mathbb{R} \cup\{+\infty\}$.

\section{The TIME-DELAYS OPERATOR}

2.1. Integration with respect to vector measures. We use the notation and results of Appendix A with $\mathbb{T}=\mathbb{R}, X=L^{2}(\Omega)$, and $\mathcal{Y}=L^{\infty}(\Omega)$. Let $\mathbf{m}$ be a $L^{\infty}(\Omega)$-valued Borel vector measure, i.e., a countably additive set function $\mathbf{m}: \mathcal{B}(\mathbb{R}) \rightarrow L^{\infty}(\Omega)$ that we assume to be of finite variation. Let B : $L^{\infty}(\Omega) \times L^{2}(\Omega) \rightarrow L^{2}(\Omega)$ be the bilinear mapping defined by $\mathbf{B}(v, u):=v u$. According to the abstract scheme described in Appendix A, we can define the integral with respect to $\mathbf{m}$. Recall that we can integrate with respect to $\mathbf{m}$ the step functions of the form $S=\sum_{i \in I} \mathbb{1}_{B_{i}} S_{i}$, where $I$ is any finite set, $B_{i} \in \mathcal{B}(\mathbb{R})$, and $S_{i} \in L^{2}(\Omega)$, according to the formula

$$
\begin{aligned}
\int S d \mathbf{m} & :=\sum_{i \in I} \mathbf{B}\left(\mathbf{m}\left(B_{i}\right), S_{i}\right) \\
& =\sum_{i \in I} \mathbf{m}\left(B_{i}\right) S_{i}
\end{aligned}
$$

which defines an element of $L^{2}(\Omega)$. This integral can be extended to the space $\mathcal{E}^{\infty}\left(\mathbb{R}, L^{2}(\Omega)\right)$ of functions $u: \mathbb{R} \rightarrow L^{2}(\Omega)$ which are uniform limit of step functions, thus defining an element of $L^{2}(\Omega)$ (see Proposition A.2. Moreover we have

$$
\left\|\int u d \mathbf{m}\right\|_{L^{2}(\Omega)} \leq \int\|u\|_{L^{2}(\Omega)} d\|\mathbf{m}\|,
$$


or, if we want to highlight the time variable, $\left\|\int u(t) d \mathbf{m}(t)\right\|_{L^{2}(\Omega)} \leq \int\|u(t)\|_{L^{2}(\Omega)} d\|\mathbf{m}\|(t)$. Recall that $C_{c}\left(\mathbb{R}, L^{2}(\Omega)\right) \subset \mathcal{E}^{\infty}\left(\mathbb{R}, L^{2}(\Omega)\right)$.

2.2. Time delays-operator associated with vector measures. The distributed delays considered in this chapter, are modeled by a family $\left(\mathbf{m}_{t}\right)_{t>0}$ of Borel vector measures $\mathbf{m}_{t}: \mathcal{B}(\mathbb{R}) \rightarrow L^{\infty}(\Omega)$ as defined above, whose total variation satisfies

$$
\left(\mathrm{M}_{1}\right) \sup _{t \geq 0}\left\|\mathbf{m}_{t}\right\|(\mathbb{R}) \leq 1 .
$$

We assume that for all $t \geq 0, \mathbf{m}_{t}$ is positive in the following sense:

$$
\left(\mathrm{M}_{2}\right) \forall t \geq 0 \quad \forall u \in \mathcal{E}^{\infty}\left(\mathbb{R}, L^{2}(\Omega)\right) \quad u \geq 0 \Longrightarrow \int u d \mathbf{m}_{t} \geq 0 .
$$

From $\left(\mathrm{M}_{1}\right)$ and $\left(\mathrm{M}_{2}\right)$, we infer that for all $t \geq 0, \mathbf{m}_{t}$ takes its values in the subset of $L^{\infty}(\Omega)$ made up of functions $v$ satisfying $0 \leq v \leq 1$. Indeed, the claim follows from

$$
0 \leq \mathbf{m}_{t}(B)=\left|\mathbf{m}_{t}(B)\right| \leq\left\|\mathbf{m}_{t}(B)\right\|_{L^{\infty}(\Omega)} \leq\left\|\mathbf{m}_{t}\right\|(B) \leq\left\|\mathbf{m}_{t}\right\|(\mathbb{R}) \leq 1
$$

for all $B \in \mathcal{B}(\mathbb{R})$.

Denote by $\mathbf{M}_{1}^{+}\left(\mathbb{R}, L^{\infty}(\Omega)\right)$ the set of positive vector Borel measures $\mathbf{m}: \mathcal{B}(\mathbb{R}) \rightarrow L^{\infty}(\Omega)$ satisfying $\|\mathbf{m}\|(\mathbb{R}) \leq 1$. We assume furthermore that

$\left(\mathrm{M}_{3}\right)$ the map

$$
\begin{aligned}
& {[0,+\infty) } \longrightarrow \mathbf{M}_{1}^{+}\left(\mathbb{R}, L^{\infty}(\Omega)\right) \\
& t \longmapsto \\
& \mathbb{1}_{(-\infty, t]} \mathbf{m}_{t}
\end{aligned}
$$

is measurable when $\mathbf{M}_{1}^{+}\left(\mathbb{R}, L^{\infty}(\Omega)\right)$ is equipped with the $\sigma$-algebra generated by the family of evaluation maps $\left(\mathscr{E}_{u}\right)_{u \in \mathcal{E}^{\infty}\left(\mathbb{R}, L^{2}(\Omega)\right)}, \mathscr{E}_{u}: \mathbf{M}_{1}^{+}\left(\mathbb{R}, L^{\infty}(\Omega)\right) \rightarrow L^{2}(\Omega)$ defined by

$$
\mathscr{E}_{u}(\mathbf{m}):=\int u d \mathbf{m} .
$$

On other word, for all $u \in \mathcal{E}^{\infty}\left(\mathbb{R}, L^{2}(\Omega)\right)$, the maps $t \mapsto \int_{-\infty}^{t} u d \mathbf{m}_{\uparrow} \sqrt{1}$ are measurable from $[0,+\infty)$ into $L^{2}(\Omega)$.

Definition 2.1. Let $T>0$ be fixed. Let $\left(\mathbf{m}_{t}\right)_{t \geq 0}$ be a family of vector measures in $\mathbf{M}_{1}^{+}\left(\mathbb{R}, L^{\infty}(\Omega)\right)$ satisfying $\left(\mathrm{M}_{1}\right)\left(\mathrm{M}_{2}\right)$ and $\left(\mathrm{M}_{3}\right)$. We call time-delays operator associated with $\left(\mathbf{m}_{t}\right)_{t \geq 0}$, the linear continuous mapping $T$ defined by:

$$
\begin{aligned}
\mathcal{T}: C_{c}\left((-\infty, T], L^{2}(\Omega)\right) & \longrightarrow L^{2}\left(0, T, L^{2}(\Omega)\right) \\
u & \longmapsto \mathcal{T} u \\
\text { where for all t in }[0, T], \mathcal{T} u(t) & =\int_{-\infty}^{t} u(s) d \mathbf{m}_{t}(s) .
\end{aligned}
$$

Note that for each $t \in[0, T], \mathcal{T} u(t)$ is well defined. Indeed, let us extend the restriction to $(-\infty, t]$ of $u \in C_{c}\left((-\infty, T], L^{2}(\Omega)\right)$ by $\widetilde{u}_{t} \in C_{c}\left(\mathbb{R}, L^{2}(\Omega)\right)$ defined as follows

$$
\widetilde{u}_{t}(s)=\left\{\begin{array}{l}
u(s) \text { for } s \in(-\infty, t], \\
\left.\left.\frac{t-s}{\delta} u(t)+u(t) \text { for } s \in\right] t, t+\delta\right], \\
0 \text { for } s \in] t+\delta,+\infty) .
\end{array}\right.
$$

Then $\mathbb{1}_{(-\infty, t]} u=\mathbb{1}_{(-\infty, t]} \widetilde{u}_{t}, \mathbb{1}_{(-\infty, t]} \widetilde{u}_{t} \in \mathcal{E}^{\infty}\left(\mathbb{R}, L^{2}(\Omega)\right)$, and

$$
\mathcal{T} u(t)=\int \mathbb{1}_{(-\infty, t]} \widetilde{u}_{t}(s) d \mathbf{m}_{t}(s)
$$

\footnotetext{
${ }^{1}$ In a standard way, we write $\int_{-\infty}^{t} u d \mathbf{m}_{t}$ for $\int \mathbb{1}_{(-\infty, t]} u d \mathbf{m}_{t}$.
} 
Furthermore, the operator $\mathcal{T}$ takes its values in $L^{2}\left(0, T, L^{2}(\Omega)\right)$ and is continuous. Indeed $t \mapsto \mathcal{T} u(t)$ is clearly measurable, $\left\|\widetilde{u}_{t}\right\|_{C_{c}\left(\mathbb{R}, L^{2}(\Omega)\right)} \leq 2\|u\|_{C_{c}\left((-\infty, T], L^{2}(\Omega)\right)}$, and

$$
\begin{aligned}
& \|\mathcal{T} u(t)\|_{L^{2}(\Omega)} \leq \int\left\|\widetilde{u}_{t}(s)\right\|_{L^{2}(\Omega)} d\left\|\mathbf{m}_{t}\right\|(s) \leq 2\|u\|_{C_{c}\left((-\infty, T], L^{2}(\Omega)\right)}, \\
& \|\mathcal{T} u\|_{L^{2}\left(0, T, L^{2}(\Omega)\right)} \leq 2 T^{\frac{1}{2}}\|u\|_{C_{c}\left((-\infty, T], L^{2}(\Omega)\right)} .
\end{aligned}
$$

For $T^{\prime}>T$, let denote by $\mathcal{T}^{\prime}$ the time-delays operator associated with $\left(\mathbf{m}_{t}\right)_{t \geq 0}$ defined on the space $C_{c}\left(\left(-\infty, T^{\prime}\right], L^{2}(\Omega)\right)$. Then, the restriction of $\mathcal{T}^{\prime}$ to $C_{c}\left((-\infty, T], L^{2}(\Omega)\right)$ is clearly the time-delays operator $\mathcal{T}$. According to this remark, we do not indicate the dependance of $\mathcal{T}$ with respect to $T$.

Lemma 2.1. Let $u \in C_{c}\left((-\infty, T], L^{2}(\Omega)\right), u \geq 0$. Assume that there exists $\bar{u} \in \mathbb{R}_{+}$such that $0 \leq u \leq \bar{u}$. Then $0 \leq \mathcal{T} u \leq \bar{u}$.

Proof. From $\left(\mathrm{M}_{2}\right)$ for all $t \in[0, T]$, we have $\mathcal{T} u(t)=\int \mathbb{1}_{(-\infty, t]} \widetilde{u}_{t}(s) d \mathbf{m}_{t}(t) \geq 0$. On the other hand, for all $t \in[0, T]$, since $\mathbf{m}_{t}((-\infty, t]) \leq 1$ in $L^{\infty}(\Omega)$, we infer that

$$
\bar{u}-\mathcal{T} u(t) \geq \mathbf{m}_{t}((-\infty, t]) \bar{u}-\mathcal{T} u(t)=\int_{-\infty}^{t}(\bar{u}-u) d \mathbf{m}_{t}=\int \mathbb{1}_{(-\infty, t]}\left(\bar{u}-\widetilde{u}_{t}\right) d \mathbf{m}_{t}
$$

with $\mathbb{1}_{(-\infty, t]}\left(\bar{u}-\widetilde{u}_{t}\right) \geq 0$, so that $\mathcal{T} u \leq \bar{u}$.

In order that the solutions to problems of the type $\left(\mathcal{P}_{n}\right)$ admit a right derivative at each $t \in[0, T]$, we assume that $\left(\mathbf{m}_{t}\right)_{t \geq 0}$ satisfies some regularity hypothesis through the operator $\mathcal{T}$, made precise in $\left(\mathrm{M}_{4}\right)$ below. Let $\eta \in C_{c}\left((-\infty, 0], L^{2}(\Omega)\right)$, absolutely continuous, and satisfying

$$
\int_{-\infty}^{0}\left\|\frac{d \eta}{d \sigma}\right\|_{L^{2}(\Omega)} d \sigma<+\infty
$$

We denote by $C_{\eta}\left((-\infty, T], L^{2}(\Omega)\right)$ the subset of $C_{c}\left((-\infty, T], L^{2}(\Omega)\right)$ made up of functions $u$ which are absolutely continuous, and whose restriction to $(-\infty, 0]$ is equal to $\eta$. Then condition $\left(\mathrm{M}_{4}\right)$ is expressed as follows:

$\left(\mathrm{M}_{4}\right)$ For all $T>0$ and all function $u$ in $C_{\eta}\left((-\infty, T], L^{2}(\Omega)\right)$, there exists a locally integrable function $\varphi_{u}:[0, T] \rightarrow \mathbb{R}_{+}$, such that

$$
\forall(s, t) \in[0, T]^{2} \quad s<t \Longrightarrow\|\mathcal{T} u(t)-\mathcal{T} u(s)\|_{L^{2}(\Omega)} \leq \int_{s}^{t} \varphi_{u}(\sigma) d \sigma .
$$

We examine below three basic examples of vector measures in $\mathbf{M}_{1}^{+}\left(\mathbb{R}, L^{\infty}(\Omega)\right)$ and time-delays operators.

\subsection{Examples of time-delays operators.}

Examples 2.1 (Multi-delays case). Consider $\left(\mathbf{m}_{t}\right)_{t \geq 0}$ given by $\mathbf{m}_{t}=\sum_{k \in \mathbb{N}} \mathbf{d}_{k} \delta_{t-\tau_{k}}$ where, for all $k \in \mathbb{N}, \tau_{k}>0, \mathbf{d}_{k} \in L^{\infty}(\Omega), \mathbf{d}_{k} \geq 0, \sum_{k \in \mathbb{N}}\left\|\mathbf{d}_{k}\right\|_{L^{\infty}(\Omega)}=1$. Clearly $\mathbf{m}_{t}$ belongs to $\mathbf{M}_{1}^{+}\left(\mathbb{R}, L^{\infty}(\Omega)\right)$. For any $u \in \mathcal{E}^{\infty}\left(\mathbb{R}, L^{2}(\Omega)\right)$, the map $t \mapsto \int_{-\infty}^{t} u(s) d \mathbf{m}_{t}$ is nothing but $t \mapsto \sum_{k \in \mathbb{N}} \mathbf{d}_{k} u\left(t-\tau_{k}\right)$ so that $\left(\mathrm{M}_{1}\right),\left(\mathrm{M}_{2}\right)$ and $\left(\mathrm{M}_{3}\right)$ are fulfilled. The operator $\mathcal{T}$ is defined for every $u \in C_{c}\left((-\infty, T], L^{2}(\Omega)\right)$ by $\mathcal{T} u(t)=\sum_{k \in \mathbb{N}} \mathbf{d}_{k} u\left(t-\tau_{k}\right)$.

Let $(s, t) \in[0, T]^{2}$ be such that $s<t$. Using Fubini-Tonelli theorem, for all $u \in C_{\eta}\left((-\infty, T], L^{2}(\Omega)\right)$ we have

$$
\begin{aligned}
\|\mathcal{T} u(t)-\mathcal{T} u(s)\|_{L^{2}(\Omega)} & \leq \sum_{k \in \mathbb{N}}\left\|\mathbf{d}_{k}\right\|_{L^{\infty}(\Omega)}\left\|u\left(t-\tau_{k}\right)-u\left(s-\tau_{k}\right)\right\|_{L^{2}(\Omega)} \\
& \leq \sum_{k \in \mathbb{N}}\left\|\mathbf{d}_{k}\right\|_{L^{\infty}(\Omega)} \int_{s-\tau_{k}}^{t-\tau_{k}}\left\|\frac{d u}{d \sigma}(\sigma)\right\|_{L^{2}(\Omega)} d \sigma \\
& =\int_{s}^{t}\left(\sum_{k \in \mathbb{N}}\left\|\mathbf{d}_{k}\right\|_{L^{\infty}(\Omega)}\left\|\frac{d u}{d \sigma}\left(\sigma-\tau_{k}\right)\right\|_{L^{2}(\Omega)}\right) d \sigma
\end{aligned}
$$


so that, $\varphi_{u}:[0, T] \rightarrow \mathbb{R}_{+}$defined by

$$
\varphi_{u}(\sigma)=\sum_{k \in \mathbb{N}}\left\|\mathbf{d}_{k}\right\|_{L^{\infty}(\Omega)}\left\|\frac{d u}{d \sigma}\left(\sigma-\tau_{k}\right)\right\|_{L^{2}(\Omega)},
$$

is suitable for satisfying $\left(\mathrm{M}_{4}\right)$. Note that $\varphi_{u}$ is the discrete convolution

$$
\left\|\frac{d u}{d \sigma}(\cdot)\right\|_{L^{2}(\Omega)} \star \sum_{k \in \mathbb{N}}\left\|\mathbf{d}_{k}\right\|_{L^{\infty}(\Omega)} \delta_{\tau_{k}}
$$

Examples 2.2 (Single delay depending on the space variable). Given a measurable map $\tau$ : $\mathbb{R}^{N} \rightarrow[0,+\infty)$ with $\#\left(\tau\left(\mathbb{R}^{N}\right)\right)<+\infty$ (i.e., with finite range), we set $\mathbf{m}_{t}=\frac{1}{\#\left(\tau\left(\mathbb{R}^{N}\right)\right)} \delta_{t-\tau(\cdot)}$. It is easily seen that $\mathbf{m}_{t}$ is a vector measure from $\mathcal{B}(\mathbb{R})$ into $L^{\infty}(\Omega)$ satisfying $\left(\mathrm{M}_{2}\right)$ and $\left(\mathrm{M}_{3}\right)$. Let us show that $\mathbf{m}_{t}$ satisfies $\left(\mathrm{M}_{1}\right)$. Indeed, we have

$$
\begin{aligned}
\left\|\mathbf{m}_{t}\right\|\left(\mathbb{R}^{N}\right) & =\sup _{I}\left\{\sum_{i \in I}\left\|\frac{1}{\#\left(\tau\left(\mathbb{R}^{N}\right)\right)} \delta_{t-\tau(\cdot)}\left(B_{i}\right)\right\|_{L^{\infty}(\Omega)}: B_{i} \in \mathcal{B}(\mathbb{R}),\left(B_{i}\right)_{i \in I} \text { finite partition of } \mathbb{R}\right\} \\
& =\sup _{I}\left\{\frac{\#\left(A_{I}\right)}{\#(\tau(\mathbb{R}))}: B_{i} \in \mathcal{B}(\mathbb{R}),\left(B_{i}\right)_{i \in I} \text { finite partition of } \mathbb{R}\right\}
\end{aligned}
$$

where $A_{I}:=\left\{i \in I: \tau^{-1}\left(t-B_{i}\right) \neq \emptyset\right\}$. For each fixed $I$, and for each $i \in A_{I}$, choose $x_{i}$ in $\mathbb{R}^{N}$ such that $\tau\left(x_{i}\right) \in t-B_{i}$, and consider the map $\Gamma_{I}: A_{I} \rightarrow \tau\left(\mathbb{R}^{N}\right)$ defined by $\Gamma_{I}(i)=\tau\left(x_{i}\right)$. From the fact that $t-B_{i}$ and $t-B_{j}$ are disjoint sets for $i \neq j$, we infer that $\Gamma_{I}$ is an injection, thus $\#\left(A_{I}\right) \leq \#\left(\tau\left(\mathbb{R}^{N}\right)\right)$ which proves the claim.

The operator $\mathcal{T}$ is then defined for all $t \in[0, T]$ and all $u \in C_{c}\left((-\infty, T], L^{2}(\Omega)\right)$ by $\mathcal{T} u(t)=$ $\frac{1}{\#\left(\tau\left(\mathbb{R}^{N}\right)\right)} u(t-\tau(\cdot))$, i.e., for a.e. $x \in \Omega, \mathcal{T} u(t)(x)=\left.\frac{1}{\#\left(\tau\left(\mathbb{R}^{N}\right)\right)} u(t-\tau(x), x)\right|^{2}$, Let us show that $\left(\mathrm{M}_{4}\right)$ is satisfied. For all $u \in C_{\eta}\left((-\infty, T], L^{2}(\Omega)\right)$ we have

$$
\begin{aligned}
& \left\|\frac{1}{\#\left(\tau\left(\mathbb{R}^{N}\right)\right)}(u(t-\tau(\cdot))-u(s-\tau(\cdot)))\right\|_{L^{2}(\Omega)} \\
& \leq \frac{1}{\#\left(\tau\left(\mathbb{R}^{N}\right)\right)} \int_{s}^{t}\left\|\frac{d u}{d \sigma}(\sigma-\tau(\cdot))\right\|_{L^{2}(\Omega)} d \sigma \\
& \leq \frac{1}{\#\left(\tau\left(\mathbb{R}^{N}\right)\right)} \int_{s}^{t}\left(\sum_{\tau_{i} \in \tau\left(\mathbb{R}^{N}\right)} \int_{\tau^{-1}\left(\tau_{i}\right) \cap \Omega}\left|\frac{d u}{d \sigma}\left(\sigma-\tau_{i}, x\right)\right|^{2} d x\right)^{\frac{1}{2}} d \sigma \\
& \leq \frac{1}{\#\left(\tau\left(\mathbb{R}^{N}\right)\right)} \int_{s}^{t} \sum_{\tau_{i} \in \tau\left(\mathbb{R}^{N}\right)}\left\|\frac{d u}{d \sigma}\left(\sigma-\tau_{i}\right)\right\|_{L^{2}(\Omega)} d \sigma
\end{aligned}
$$

so that $\varphi_{u}$ given by

$$
\varphi_{u}(\sigma)=\frac{1}{\#\left(\tau\left(\mathbb{R}^{N}\right)\right)} \sum_{\tau_{i} \in \tau\left(\mathbb{R}^{N}\right)}\left\|\frac{d u}{d \sigma}\left(\sigma-\tau_{i}\right)\right\|_{L^{2}(\Omega)}
$$

is suitable to check $\left(\mathrm{M}_{4}\right)$. Note that $\varphi_{u}$ is the discrete convolution

$$
\left\|\frac{d u}{d \sigma}(\cdot)\right\|_{L^{2}(\Omega)} \star \frac{1}{\#\left(\tau\left(\mathbb{R}^{N}\right)\right)} \sum_{\tau_{i} \in \tau\left(\mathbb{R}^{N}\right)} \delta_{\tau_{i}} .
$$

Examples 2.3 (Delays distributed by a diffuse delays kernel). Consider $\left(\mathbf{m}_{t}\right)_{t \geq 0}$ given by $\mathbf{m}_{t}=$ $\mathcal{K}_{t}(\cdot, \tau) d \tau$ where

(i) $\forall \tau \in \mathbb{R}_{+}, \quad \forall t \geq 0 \quad \mathcal{K}_{t}(\cdot, \tau)=\mathcal{K}(\cdot, t-\tau)$;

(ii) $\forall \sigma \in \mathbb{R} \quad \mathcal{K}(\cdot, \sigma) \in L^{\infty}(\Omega)$;

(iii) $\int_{-\infty}^{+\infty}\|\mathcal{K}(\cdot, \sigma)\|_{L^{\infty}(\Omega)} d \sigma=1$;

\footnotetext{
${ }^{2}$ To shorten the notation, we sometimes write $v(t, x)$ for $v(t)(x)$ for the functions $v \in L^{2}\left(0, T, L^{2}(\Omega)\right)$.
} 
(iv) $\forall \sigma \in \mathbb{R} \quad \mathcal{K}(x, \sigma) \geq 0$ a.e. in $\Omega$;

(v) $\forall \sigma \in(-\infty, 0) \quad \mathcal{K}(\cdot, \sigma)=0$.

It is easy to show that $\mathbf{m}_{t}$ satisfies $\left(\mathrm{M}_{1}\right)$ and $\left(\mathrm{M}_{2}\right)$. An example of kernel $\mathcal{K}$ is given by $\mathcal{K}(\cdot, \sigma)=\mathbf{d}(\cdot) K(\sigma)$

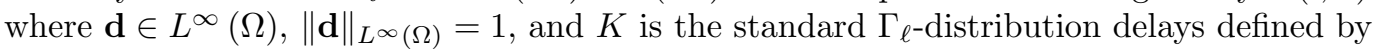

$$
K(\sigma)=\left\{\begin{array}{cc}
\frac{\gamma \sigma^{\ell-1} \exp (-\gamma \sigma)}{(\ell-1) !} & \text { if } \sigma \geq 0 \\
0 & \text { if } \sigma<0
\end{array}\right.
$$

with $\ell \in \mathbb{N}^{*}$ and $\gamma>0$.

For any $u \in \mathcal{E}^{\infty}\left(\mathbb{R}, L^{2}(\Omega)\right)$, the map $t \mapsto \int_{-\infty}^{t} u(s) \mathcal{K}(\cdot, t-s) d s=\int_{0}^{+\infty} u(t-s) \mathcal{K}(\cdot, s) d s$ is clearly measurable so that $\left[\left(\mathrm{M}_{3}\right)\right.$ is satisfied. For all $u \in C_{c}\left((-\infty, T], L^{2}(\Omega)\right)$ we have

$$
\forall t \in[0, T], \mathcal{T} u(t)=\int_{-\infty}^{t} u(s) \mathcal{K}(\cdot, t-s) d s=\int_{0}^{+\infty} u(t-s) \mathcal{K}(\cdot, s) d s=u \star \mathcal{K}(\cdot, \cdot)(t) .
$$

An elementary calculation shows that $\varphi_{u}=\left\|\frac{d u}{d \sigma}(\cdot)\right\|_{L^{2}(\Omega)} \star\|\mathcal{K}(\cdot, \tau)\|_{L^{\infty}(\Omega)} d \tau$ is suitable to check $\left(\mathrm{M}_{4}\right)$ Indeed, for all $u \in C_{\eta}\left((-\infty, T], L^{2}(\Omega)\right)$ we have

$$
\begin{aligned}
& \left\|\int_{0}^{+\infty} \mathcal{K}(\cdot, \tau) u(t-\tau) d \tau-\int_{0}^{+\infty} \mathcal{K}(\cdot, \tau) u(s-\tau) d \tau\right\|_{L^{2}(\Omega)} \\
& \leq \int_{0}^{+\infty}\|\mathcal{K}(\cdot, \tau)\|_{L^{\infty}(\Omega)}\|u(t-\tau)-u(s-\tau)\|_{L^{2}(\Omega)} d \tau \\
& \leq \int_{0}^{+\infty}\|\mathcal{K}(\cdot, \tau)\|_{L^{\infty}(\Omega)}\left(\int_{s-\tau}^{t-\tau}\left\|\frac{d u}{d \sigma}(\sigma)\right\|_{L^{2}(\Omega)} d \sigma\right) d \tau \\
& =\int_{s}^{t}\left(\int_{0}^{+\infty}\|\mathcal{K}(\cdot, \tau)\|_{L^{\infty}(\Omega)}\left\|\frac{d u}{d \sigma}(\sigma-\tau)\right\|_{L^{2}(\Omega)} d \tau\right) d \sigma,
\end{aligned}
$$

and the claim follows by setting $\varphi_{u}(\sigma)=\int_{0}^{+\infty}\left\|\frac{d u}{d \sigma}(\sigma-\tau)\right\|_{L^{2}(\Omega)}\|\mathcal{K}(\cdot, \tau)\|_{L^{\infty}(\Omega)} d \tau$.

Remark 2.1. Denote by $\mathcal{P}_{+}(\mathbb{R})$ the set of all probability measures on $\mathbb{R}$ concentrated on $\mathbb{R}_{+}$. One can express the functions $\varphi_{u}$ obtained in Example 2.1, 2.2 and 2.3 in an unified way: there exists $\mu \in \mathcal{P}_{+}(\mathbb{R})$ such that

$$
\varphi_{u}=\left\|\frac{d u}{d \sigma}(\cdot)\right\|_{L^{2}(\Omega)} \star \mu,
$$

i.e., such that for all $\sigma \in[0, T], \varphi_{u}(\sigma)=\int_{\mathbb{R}}\left\|\frac{d u}{d \sigma}(\sigma-\tau)\right\|_{L^{2}(\Omega)} d \mu(\tau)$.

In Example 2.1 $\mu=\sum_{k \in \mathbb{N}}\left\|\mathbf{d}_{k}\right\|_{L^{\infty}(\Omega)} \delta_{\tau_{k}}$, in Example 2.2 $\mu=\frac{1}{\#\left(\tau\left(\mathbb{R}^{N}\right)\right)} \sum_{\tau_{i} \in \tau\left(\mathbb{R}^{N}\right)} \delta_{\tau_{i}}$, and in Example $2.3, \mu=\|\mathcal{K}(\cdot, \tau)\|_{L^{\infty}(\Omega)} d \tau$.

In Section 4, we strengthen condition $\left(\mathrm{M}_{4}\right)$ by condition $\left(\mathrm{M}_{4}\right)$ below:

$\left(\mathrm{M}_{4}\right)$ for all $T>0$ there exists $\mu \in \mathcal{P}_{+}(\mathbb{R})$ such that all function $u$ in $C_{\eta}\left((-\infty, T], L^{2}(\Omega)\right)$,

$$
\forall(s, t) \in[0, T]^{2} \quad s<t \Longrightarrow\|\mathcal{T} u(t)-\mathcal{T} u(s)\|_{L^{2}(\Omega)} \leq \int_{s}^{t}\left(\left\|\frac{d u}{d \sigma}(\cdot)\right\|_{L^{2}(\Omega)} \star \mu\right)(\sigma) d \sigma .
$$

Note that the integral in the second member of $\left(\mathrm{M}_{4}^{\prime}\right)$ if finite as stated in the lemma below.

Lemma 2.2. Assume $\left(\mathrm{M}_{4}^{\prime}\right)$. Then for all $T>0$ and all $u \in C_{\eta}\left((-\infty, T], L^{2}(\Omega)\right)$, Tu is absolutely continuous and

$$
\int_{0}^{T}\left\|\frac{d \mathcal{T} u}{d \sigma}(\sigma)\right\|_{L^{2}(\Omega)} d \sigma \leq \int_{-\infty}^{0}\left\|\frac{d \eta}{d \sigma}(\sigma)\right\|_{X} d \sigma+\int_{0}^{T}\left\|\frac{d u}{d \sigma}(\sigma)\right\|_{X} d \sigma
$$


Proof. Fix $T>0$ and $u \in C_{\eta}\left((-\infty, T], L^{2}(\Omega)\right)$. For any finite partition $\left(s_{i}, t_{i}\right)_{i \in I}$ of $[0, T]$, from $\left(\mathrm{M}_{4}^{\prime}\right)$ and Fubini-Tonelli theorem, we have

$$
\begin{aligned}
\sum_{i \in I}\left\|\mathcal{T} u\left(t_{i}\right)-\mathcal{T} u\left(s_{i}\right)\right\|_{L^{2}(\Omega)} & \leq \sum_{i \in I} \int_{s_{i}}^{t_{i}}\left(\left\|\frac{d u}{d \sigma}(\cdot)\right\|_{X} \star \mu\right)(\sigma) d \sigma \\
& \leq \int_{0}^{T}\left(\left\|\frac{d u}{d \sigma}(\cdot)\right\|_{X} \star \mu\right)(\sigma) d \sigma \\
& =\int_{\mathbb{R}_{+}}\left(\int_{0}^{T}\left\|\frac{d u}{d \sigma}(\sigma-\tau)\right\|_{X} d \sigma\right) d \mu(\tau) \\
& =\int_{\mathbb{R}_{+}}\left(\int_{-\tau}^{T-\tau}\left\|\frac{d u}{d \sigma}(\sigma)\right\|_{X} d \sigma\right) d \mu(\tau) \\
& \leq \int_{\mathbb{R}_{+}}\left(\int_{-\infty}^{0}\left\|\frac{d \eta}{d \sigma}(\sigma)\right\|_{X} d \sigma+\int_{0}^{T}\left\|\frac{d u}{d \sigma}(\sigma)\right\|_{X} d \sigma\right) d \mu(\tau) \\
& =\int_{-\infty}^{0}\left\|\frac{d \eta}{d \sigma}(\sigma)\right\|_{X} d \sigma+\int_{0}^{T}\left\|\frac{d u}{d \sigma}(\sigma)\right\|_{X} d \sigma
\end{aligned}
$$

which is finite since $u \in C_{\eta}\left((-\infty, T], L^{2}(\Omega)\right)$ and because $\eta$ satisfies 2.4$)$. The claim follows by taking the supremum on all the finite partitions of $[0, T]$.

\section{REACTION DifFusion PROBlEMS ASSOCIATED With CONVEX FUnCTIONALS OF THE CALCUlus OF} VARIATIONS AND DCP-STRUCTURED REACTION FUNCTIONALS

We are mainly concerned with sequences of delays reaction-diffusion problems of the form

$$
\left(\mathcal{P}_{\mathcal{T}, \eta}\right)\left\{\begin{array}{l}
\frac{d u}{d t}(t)+D \Phi(u(t))=F(t, u(t), \mathcal{T} u(t)) \text { for a.e. } t \in(0, T) \\
u(t)=\eta(t) \text { for all } t \in(-\infty, 0]
\end{array}\right.
$$

where $\mathcal{T}$ is a time-delays operator as defined in Definition 2.1. associated with a family $\left(\mathbf{m}_{t}\right)_{t>0}$ of Borel vector measures satisfying $\left(\mathrm{M}_{1}\right),\left(\mathrm{M}_{2}\right),\left(\mathrm{M}_{3}\right)$. We sometimes assume that $\left(\mathbf{m}_{t}\right)_{t>0}$ fulfills one of the additional conditions $\left(\mathrm{M}_{4}\right)$ or $\left(\mathrm{M}_{4}^{\prime}\right)$ with respect to the absolutely continuous function $\eta \in$ $C_{c}\left((-\infty, 0], L^{2}(\Omega)\right)$. Furthermore we assume that $\eta(0) \in \overline{\operatorname{dom}(\partial \Phi)}$, where $\partial \Phi$ is the subdifferential of a standard convex functional $\Phi: L^{2}(\Omega) \rightarrow \mathbb{R} \cup\{+\infty\}$ defined by

$$
\Phi(u)= \begin{cases}\int_{\Omega} W(x, \nabla u(x)) d x+\frac{1}{2} \int_{\partial \Omega} a_{0} u^{2} d \mathcal{H}_{N-1}-\int_{\partial \Omega} \phi u d \mathcal{H}_{N-1} & \text { if } u \in H^{1}(\Omega), \\ +\infty & \text { otherwise }\end{cases}
$$

where $a_{0} \in L_{\mathcal{H}_{N-1}}^{\infty}(\partial \Omega)$ with $\begin{cases}a_{0} \geq 0 & \mathcal{H}_{N-1} \text {-a.e. in } \partial \Omega \\ \exists \sigma>0 & a_{0} \geq \sigma \quad \mathcal{H}_{N-1} \text {-a.e. in } \Gamma \subset \partial \Omega \text { with } \mathcal{H}_{N-1}(\Gamma)>0,\end{cases}$

and $\phi \in L_{\mathcal{H}_{N-1}}^{2}(\partial \Omega)$. The density $W: \mathbb{R}^{N} \times \mathbb{R}^{N} \rightarrow \mathbb{R}$ is a Borel measurable function which satisfies the following conditions:

$\left(\mathrm{D}_{1}\right)$ there exist $\alpha>0$ and $\beta>0$ such that for a.e. $x \in \mathbb{R}^{N}$ and every $\xi \in \mathbb{R}^{N}$

$$
\alpha|\xi|^{2} \leq W(x, \xi) \leq \beta\left(1+|\xi|^{2}\right),
$$

$\left(\mathrm{D}_{2}\right)$ for a.e. $x \in \mathbb{R}^{N}, \xi \mapsto W(x, \xi)$ is a Gâteaux differentiable and convex function (we denote by $D_{\xi} W(x, \cdot)$ its Gâteaux derivative), and

$$
D_{\xi} W(x, 0)=0 .
$$

The Gâteaux-differentiability assumption on the density $W$ is made to simplify the notation in the proofs. From the second estimate, we infer that if $u \in H^{1}(\Omega)$, then the function $D_{\xi} W(\cdot, \nabla u)$ belongs to 
$L^{2}(\Omega)^{N}$. Furthermore it is easy to establish that there exists a constant $C\left(C_{\text {trace }}\right)>0$ which depends on the constant $C_{\text {trace }}$ of continuity of the trace operator from $H^{1}(\Omega)$ into $L^{2}(\partial \Omega)$, such that

$$
\inf _{v \in L^{2}(\Omega)} \Phi(v) \geq-C\left(C_{\text {trace }}\right)\|\phi\|_{L_{\mathcal{H}_{N-1}}^{2}(\partial \Omega)}^{2} .
$$

For a proof refer to [1]. The subdifferential $A=\partial \Phi$ of $\Phi$ (actually its Gâteaux derivative $D \Phi$ ), whose domain captures the boundary condition, is given by:

$$
\begin{aligned}
\operatorname{dom}(A) & =\left\{v \in H^{1}(\Omega): \operatorname{div} D_{\xi} W(\cdot, \nabla v) \in L^{2}(\Omega), a_{0} v+D_{\xi} W(\cdot, \nabla v) \cdot \mathbf{n}=\phi \text { on } \partial \Omega\right\} \\
A(v) & =-\operatorname{div} D_{\xi} W(\cdot, \nabla v) \text { for } v \in \operatorname{dom}(A)
\end{aligned}
$$

where $\alpha_{0} v+D_{\xi} W(\cdot, \nabla v) \cdot \mathbf{n}$ must be taken in the trace sense. For a proof, refer to [2, Lemma 3.1].

Assume that $\phi=0$. Let $\Gamma$ be a subset of $\partial \Omega$ with positive $\mathcal{H}_{N-1}$-Hausdorff measure and define $a_{0}$ in $\mathbb{R}_{+} \cup\{+\infty\}$ in the following way:

$$
a_{0}(x)= \begin{cases}0 & \text { if } x \in \partial \Omega \backslash \Gamma \\ +\infty & \text { if } x \in \Gamma\end{cases}
$$

Then, the integral $\int_{\partial \Omega} a_{0} u^{2} d \mathcal{H}_{N-1}$ may be considered as a penalization which forces the function $u$ to belongs to $H_{\Gamma}^{1}(\Omega)=\left\{u \in H^{1}(\Omega): u=0\right.$ on $\left.\Gamma\right\}$. By convention used all along the paper, the functional $\Phi$ becomes now

$$
\Phi(u)= \begin{cases}\int_{\Omega} W(x, \nabla u(x)) d x & \text { if } u \in H_{\Gamma}^{1}(\Omega), \\ +\infty & \text { otherwise. }\end{cases}
$$

For a justification of this convention, refer to [2, Remark 4.2]. The subdifferential $A=\partial \Phi(=D \Phi)$ of $\Phi$ contains now the homogeneous Dirichlet-Neumann boundary conditions and is given by:

$$
\begin{aligned}
\operatorname{dom}(A) & =\left\{v \in H_{\Gamma}^{1}(\Omega): \operatorname{div} D_{\xi} W(\cdot, \nabla v) \in L^{2}(\Omega), D_{\xi} W(\cdot, \nabla v) \cdot \mathbf{n}=0 \text { on } \partial \Omega \backslash \Gamma\right\} \\
A(v) & =-\operatorname{div} D_{\xi} W(\cdot, \nabla v) \text { for } v \in \operatorname{dom}(A) .
\end{aligned}
$$

3.1. The class of DCP-structured reaction functionals. The delays reaction-diffusion problems modeling a wide class of applications with a potential mathematical tractability in the homogenization framework (periodic or stochastic), involve a special class of reaction functionals that we define below.

Definition 3.1. A functional $F:[0,+\infty) \times L^{2}(\Omega) \times L^{2}(\Omega) \rightarrow \mathbb{R}^{\Omega}$ is called a DCP-structured reaction functional, if there exists a measurable function $f:[0,+\infty) \times \mathbb{R}^{N} \times \mathbb{R} \times \mathbb{R} \rightarrow \mathbb{R}$ such that for all $t \in[0,+\infty)$ and all $(u, v) \in L^{2}(\Omega) \times L^{2}(\Omega), F(t, u, v)(x)=f(t, x, u(x), v(x))$, fulfilling the following structure conditions:

with the assumptions:

$$
f\left(t, x, \zeta, \zeta^{\prime}\right)=r(t, x) \odot h\left(\zeta^{\prime}\right) \cdot g(\zeta)+q(t, x)
$$

- $g, h: \mathbb{R} \rightarrow \mathbb{R}^{l}$ are locally Lipschitz continuous functions;

- for all $T>0, r \in L^{\infty}\left([0, T] \times \mathbb{R}^{N}, \mathbb{R}^{l}\right)$;

- for all $T>0, q \in L^{2}\left(0, T, L_{l o c}^{2}\left(\mathbb{R}^{N}\right)\right)$.

Furthermore $f$ must satisfy the following condition (DCP) (for Delays Comparison Principle):

(DCP) there exist $\bar{f}:[0,+\infty) \times \mathbb{R} \rightarrow \mathbb{R}_{+}$and $\bar{\rho} \in \mathbb{R}_{+}^{*}$ such that the ordinary differential equation

$$
\overline{\mathrm{ODE}}\left\{\begin{array}{l}
\bar{y}^{\prime}(t)=\bar{f}(t, \bar{y}(t)) \text { for a.e. } t \in[0,+\infty) \\
\bar{y}(0)=\bar{\rho}
\end{array}\right.
$$

admits at least a solution $\bar{y}$ such that for all $T>0$, for a.e. $(t, x) \in(0, T) \times \mathbb{R}^{N}$ and for all $\zeta^{\prime} \in[0, \bar{y}(T)]$

$$
0 \leq f\left(t, x, 0, \zeta^{\prime}\right) \quad \text { and } \quad f\left(t, x, \bar{y}(t), \zeta^{\prime}\right) \leq \bar{f}(t, \bar{y}(t))
$$

\footnotetext{
${ }^{3}$ Using the coordinates of $r, g$ and $h$ we have $f\left(t, x, \zeta, \zeta^{\prime}\right)=\sum_{i=1}^{l} r_{i}(t, x) g_{i}(\zeta) h_{i}\left(\zeta^{\prime}\right)+q(t, x)$.
} 
The functional $F$ is referred to as a DCP-structured reaction functional associated with $(r, g, h, q)$ and $f$ as a DCP-structured reaction function associated with $(r, g, h, q)$. If furthermore, for all $T>0, r \in$ $W^{1,1}\left(0, T, L_{\text {loc }}^{2}\left(\mathbb{R}^{N}, \mathbb{R}^{l}\right)\right)$ and $q \in W^{1,1}\left(0, T, L_{\text {loc }}^{2}\left(\mathbb{R}^{N}\right)\right)$, the map $F$ is referred to as a regular DCPstructured reaction functional and $f$ as a regular DCP-structured reaction function.

Remark 3.1. 1) Since $\bar{y}$ is non decreasing, for any $T>0$ we have $0<\bar{\rho}=\bar{y}(0) \leq \bar{y}(T)$.

2) The regularity of DCP-structured reaction functional is unnecessary to assert existence of a bounded solution to our problems. It will be invoked for proving the following additional regularity: when furthermore $\eta$ satisfies (2.4) and $\left(\mathbf{m}_{t}\right)_{t>0}$ satisfies $\left(\mathrm{M}_{4}\right)$, the solution $u$ of $\left(\mathcal{P}_{\mathcal{T}, \eta}\right)$ satisfies $u(t) \in$ $\operatorname{dom}(D \Phi)$ for all $t \in(0, T)$ and possesses a right derivative at each $t \in[0, T)$.

3 ) It is worth noting that for each fixed $\zeta^{\prime}$ in $[0, \bar{y}(T)]$, the function $\zeta \mapsto f\left(t, x, \zeta, \zeta^{\prime}\right)$ is a CP-structured reaction functional associated with $\left(r \odot h\left(\zeta^{\prime}\right), g, q\right)$ in the sense of [2, Definition 3.1] (see Definition B.1, where $\underline{\rho}=0$ and $\underline{f}=0$.

4) According to (DCP) we show in Theorem 3.1, that, if the history function $\eta$ satisfies $0 \leq \eta \leq \bar{\rho}$, then $\left(\mathcal{P}_{\tau, \eta}\right)$ admits a unique solution $u$ satisfying $0 \leq u(t) \leq \bar{y}(T)$ for all $t \in[0, T]$.

3.2. Some examples of DCP-structured reaction functions coming from ecology and biology models.

\section{Examples 3.1. Example derived from vector disease model: the Cooke model.}

$$
f\left(t, x, \zeta, \zeta^{\prime}\right)=a(t, x) \zeta^{\prime}(1-\zeta)-b(t, x) \zeta
$$

where $a>0, b>0$ belong to $W^{1,1}\left(0, T, L_{\text {loc }}^{2}\left(\mathbb{R}^{N}\right)\right) \cap L^{\infty}\left([0, T] \times \mathbb{R}^{N}\right)$ for all $T>0$. Clearly the function $f$ satisfies the structure condition of regular DCP-structured reaction function with $l=2$ and

$$
r(t, x)=(a(t, x),-b(t, x)), h\left(\zeta^{\prime}\right)=\left(\zeta^{\prime}, 1\right), g(\zeta)=(1-\zeta, \zeta) .
$$

Let us show that (DCP) is fulfilled. Take $\bar{f}=0$, and for $\bar{\rho}$, any real number greater or equal to 1 . We have $\bar{y}=\bar{\rho}$ and, for all $\zeta^{\prime} \geq 0$,

$$
0 \leq a(t, x) \zeta^{\prime}=f\left(t, x, 0, \zeta^{\prime}\right) \quad \text { and } \quad f\left(t, x, \bar{y}(t), \zeta^{\prime}\right)=f\left(t, x, \bar{\rho}, \zeta^{\prime}\right) \leq 0=\bar{f}(t, \bar{y}(t)) .
$$

This example of reaction function corresponds to the diffusive vector disease model of Cooke (see [21, 22] in the case of single delay):

$$
\left\{\begin{array}{l}
\frac{d u}{d t}(t)+D \Phi(u(t))=a(t, x)(1-u(t)) \int_{-\infty}^{t} u(s) d \mathbf{m}_{t}(s)-b(t, \cdot) u(t) \text { for a.e. } t \in(0, T) \\
u(t)=\eta(t) \text { for all } t \in(-\infty, 0]
\end{array}\right.
$$

where

- $u(t, x)$ denotes the density of the host (i.e. infected) population,

- $1-u(t, x)$ the density of uninfected population,

- when $\mathbf{m}_{t}=\delta_{t-\tau}$, then $\tau$ denotes the incubation period before the disease agent can infect the host.

A choice of more general measures $\mathbf{m}_{t}$ corresponds to more complex incubation processes. Then if the history function $\eta$ satisfies $0 \leq \eta \leq \bar{\rho}$ for some positive real number $\bar{\rho} \geq 1$, we prove in Theorem 3.1. that the problem admits a unique solution in $C\left((-\infty, T], L^{2}(\Omega)\right)$ satisfying $0 \leq u \leq \bar{\rho}$. If moreover $\eta$ satisfies 2.4 and $\left(\mathbf{m}_{t}\right)_{t \geq 0}$ satisfies $\left(\mathrm{M}_{4}\right)$ then $u$ is regular in the sense of Remark 3.1 2).

Examples 3.2. Example derived from food limited models: the delay logistic equation with immigration (or stocking).

$$
f\left(t, x, \zeta, \zeta^{\prime}\right)=a(t, x) \zeta\left(1-\frac{\zeta^{\prime}}{K_{c a r}}\right)+q(t, x)
$$

where $0<a(t, x) \leq \bar{a}, K>0$, and $0 \leq q(t, x) \leq \bar{q}$. Assume that for all $T>0, a, b$ and $q$ belong to $W^{1,1}\left(0, T, L_{\text {loc }}^{2}\left(\mathbb{R}^{N}\right)\right)$. Then the function $f$ checks either of the two structures of regular DCP-structured reaction functions with $l=1$ or $l=2$. Indeed, with $l=1$ take

$$
r(t, x)=a(t, x), g(\zeta)=\zeta, h\left(\zeta^{\prime}\right)=\left(1-\frac{\zeta^{\prime}}{K_{\text {car }}}\right) .
$$


We can also write $f\left(t, x, \zeta, \zeta^{\prime}\right)=a(t, x) \zeta-a(t, x) \frac{\zeta \zeta^{\prime}}{K_{c a r}}+q(t, x)$, so that $f$ satisfies the structure condition with $l=2$ and

$$
r(t, x)=\left(a(t, x),-\frac{a(t, x)}{K_{c a r}}\right), h\left(\zeta^{\prime}\right)=\left(1, \zeta^{\prime}\right), g(\zeta)=(\zeta, \zeta) .
$$

Let us show that $f$ fulfills (DCP) Take $\bar{f}(t, \zeta)=\bar{a} \zeta+\bar{q}$, and for $\bar{\rho}$, take any positive real number. Then $\bar{y}(t)=\left(\bar{\rho}+\frac{\bar{q}}{\bar{a}}\right) \exp (\bar{\alpha} t)-\frac{\bar{q}}{\bar{a}}$ for all $t \in[0,+\infty)$. Thus for all $\zeta^{\prime} \in\left[0,\left(\bar{\rho}+\frac{\bar{q}}{\bar{\alpha}}\right) \exp (\bar{\alpha} T)-\frac{\bar{q}}{\bar{\alpha}}\right]$ we have

$$
\begin{aligned}
0 \leq f\left(t, x, 0, \zeta^{\prime}\right) & =q(t, x) \\
\text { and } f\left(t, x, \bar{y}(t), \zeta^{\prime}\right) & =a(t, x) \bar{y}(t)-a(t, x) \frac{\bar{y}(t) \zeta^{\prime}}{K_{c a r}}+q(t, x) \leq \overline{a y}(t)+\bar{q}=\bar{f}(t, \bar{y}(t)) .
\end{aligned}
$$

This regular DCP-structured reaction function corresponds to the diffusive delay logistic equation with immigration, modeling the evolution of a population density with resource regeneration time, growth rate $a$, and carrying capacity $K_{c a r}$ :

$$
\left\{\begin{array}{l}
\frac{d u}{d t}(t)+D \Phi(u(t))=a(t, \cdot) u(t)\left(1-\frac{1}{K_{c a r}} \int_{-\infty}^{t} u(\tau) d \mathbf{m}_{t}(\tau)\right)+q(t, \cdot) \text { for a.e. } t \in(0, T) \\
u(t)=\eta(t) \text { for all } t \in(-\infty, 0] .
\end{array}\right.
$$

We prove in Theorem 3.1, that if the history function $\eta$ satisfies $0 \leq \eta \leq \bar{\rho}$ for some $\bar{\rho}>0$, then this problem admits a unique solution $u$ in $C\left((-\infty, T], L^{2}(\Omega)\right)$ with $0 \leq u \leq\left(\bar{\rho}+\frac{\bar{q}}{\bar{a}}\right) \exp (\bar{\alpha} T)-\frac{\bar{q}}{\bar{a}}$. If moreover $\eta$ satisfies (2.4) and $\left(\mathbf{m}_{t}\right)_{t>0}$ satisfies $\left(\mathrm{M}_{4}\right)$, then $u$ is regular in the sense of Remark 3.1 2),

Examples 3.3. Example derived from haematopoiesis : the Wazewska-Czyziewska and Lasota model.

$$
f\left(t, x, \zeta, \zeta^{\prime}\right)=-\mu(t, x) \zeta+P(t, x) \exp \left(-\gamma \zeta^{\prime}\right)
$$

with $\mu>0, P>0$, and $\gamma>0$. We assume that for all $T>0, \mu$ and $P$ belong to $W^{1,1}\left(0, T, L_{\text {loc }}^{2}\left(\mathbb{R}^{N}\right)\right) \cap$ $L^{\infty}([0, T] \times \Omega)$. The function $f$ clearly satisfies the structure condition of regular DCP-structured reaction functions with $l=2$ and

$$
r(t, x)=(-\mu(t, x), P(t, x)), h\left(\zeta^{\prime}\right)=\left(1, \exp \left(-\gamma \zeta^{\prime}\right)\right), g(\zeta)=(\zeta, 1) .
$$

Assume furthermore that $\sup _{(t, x) \in[0,+\infty) \times \mathbb{R}^{N}} \frac{P(t, x)}{\mu(t, x)}<+\infty$. Therefore, noticing that $f\left(t, x, 0, \zeta^{\prime}\right) \geq 0$ and that for all $\zeta^{\prime} \geq 0, f\left(t, x, \rho, \zeta^{\prime}\right) \leq 0$ for $\rho \geq \sup _{(t, x) \in[0,+\infty) \times \mathbb{R}^{N}} \frac{P(t, x)}{\mu(t, x)}$, we can easily show that $f$ satisfies (DCP) Indeed, take $\bar{f}=0$ and, for $\bar{\rho}$, any positive real number satisfying

$$
\bar{\rho} \geq \sup _{(t, x) \in[0,+\infty) \times \mathbb{R}^{N}} \frac{P(t, x)}{\mu(t, x)} .
$$

Such a reaction function corresponds to the diffusive Wazewska-Czyziewska and Lasota model (see [21, 22$]$ in the case of single delay)

$$
\left\{\begin{array}{l}
\frac{d u}{d t}(t)+D \Phi(u(t))=-\mu(t, x) u(t)+P(t, \cdot) \exp \left(-\gamma \int_{-\infty}^{t} u(s) d \mathbf{m}_{t}(s)\right) \text { for a.e. } t \in(0, T) \\
u(t)=\eta(t) \text { for all } t \in(-\infty, 0]
\end{array}\right.
$$

where

- $u(t, x)$ is the number of red-blood cell at time $t$ located at $x$,

- $\mu(t, x)$ is the probability of death of red-blood cells,

- $P$ and $\gamma$ are two coefficients related to the production of red-blood cells per unit time.

When $\tau$ is the time required to produce a red-blood, we take $\mathbf{m}_{t}=\delta_{t-\tau}$. A choice of more general measures $\mathbf{m}_{t}$ corresponds to more complex regeneration time. Then if the history function $\eta$ satisfies $0 \leq \eta \leq \bar{\rho}$ for some positive real number $\bar{\rho}$ satisfying (3.4), we prove in Theorem 3.1. that the problem admits a unique solution $u$ in $C\left((-\infty, T], L^{2}(\Omega)\right)$ satisfying $0 \leq u \leq \bar{\rho}$, and if $\eta$ satisfies (2.4) and $\left(\mathbf{m}_{t}\right)_{t \geq 0}$ satisfies $\left(\mathrm{M}_{4}\right)$ then $u$ is regular in the sense of Remark 3.1 2), 
Examples 3.4. Example derived from haematopoiesis: the Mackey-Glass model.

$$
f\left(t, x, \zeta, \zeta^{\prime}\right)=-a(t, x) \zeta+b(t, x) \frac{\zeta^{\prime}}{1+\zeta^{\prime m}}
$$

where $a>0, b>0$, and $m>1$. We assume that for all $T>0, a$ and $b$ belong to $W^{1,1}\left(0, T, L_{\text {loc }}^{2}\left(\mathbb{R}^{N}\right)\right) \cap$ $L^{\infty}\left([0, T] \times \mathbb{R}^{N}\right)$. The function $f$ satisfies the structure condition of regular DCP-structured reaction functions with $l=2$ and

$$
r(t, x)=(-a(t, x), b(t, x)), h\left(\zeta^{\prime}\right)=\left(1, \frac{\zeta^{\prime}}{1+\zeta^{\prime} m}\right), g(\zeta)=(\zeta, 1) .
$$

Assume moreover that $\sup _{(t, x) \in[0,+\infty) \times \mathbb{R}^{N}} \frac{b(t, x)}{a(t, x)}<+\infty$, and check that (DCP) is satisfied. We have

$$
0 \leq f\left(t, x, 0, \zeta^{\prime}\right)=b(t, x) \frac{\zeta^{\prime}}{1+\zeta^{\prime m}}
$$

On the other hand, set $\bar{f}=0$ and take

$$
\bar{\rho} \geq \sup _{(t, x) \in[0,+\infty) \times \mathbb{R}^{N}} \frac{b(t, x)}{a(t, x)} h_{2}\left(\left(\frac{1}{m-1}\right)^{\frac{1}{m}}\right)
$$

where $h_{2}\left(\zeta^{\prime}\right)=\frac{\zeta^{\prime}}{1+\zeta^{\prime m}}$. An elementary calculation shows that $\sup _{\zeta^{\prime} \geq 0} h_{2}\left(\zeta^{\prime}\right)=h_{2}\left(\left(\frac{1}{m-1}\right)^{\frac{1}{m}}\right)$. Then for all $\zeta^{\prime} \geq 0, \bar{y}=\bar{\rho}$ satisfies

$$
f\left(t, x, \bar{y}(t), \zeta^{\prime}\right)=f\left(t, x, \bar{\rho}, \zeta^{\prime}\right) \leq 0=\bar{f}(t, \bar{y}(t)) .
$$

This example of reaction function corresponds to the diffusive Mackey-Glass model whose reaction term is proposed to model the production of white blood cells (see [5, 21, 22] in the case of a single delay):

$$
\left\{\begin{array}{l}
\frac{d u}{d t}(t)+D \Phi(u(t))=-a(t, x) u(t)+b(t, \cdot) \frac{\int_{-\infty}^{t} u(s) d \mathbf{m}_{t}(s)}{1+\left(\int_{-\infty}^{t} u(s) d \mathbf{m}_{t}(s)\right)^{m}} \text { for a.e. } t \in(0, T) \\
u(t)=\eta(t) \text { for all } t \in(-\infty, 0]
\end{array}\right.
$$

where

- $u(t, x)$ denotes the concentration of white-blood cells at time $t$ located at $x$ in circulating blood,

- the term $-a(t, x) u(t, x)$ represents the reduction of white-blood cells at time $t$, located at $x$,

- when $\mathbf{m}_{t}=\delta_{t-\tau}$, the term $b(t, \cdot) \frac{u(t-\tau)}{1+u(t-\tau)^{\prime m}}$ models the production of white-blood cells after a delay $\tau$ before the marrow releases further cells to replenish the deficiency.

As in the previous example, if the history function $\eta$ satisfies $0 \leq \eta \leq \bar{\rho}$ for some positive real number $\bar{\rho}$ satisfying $[3.5)$, then the problem admits a unique solution in $\bar{C}\left((-\infty, T], L^{2}(\Omega)\right)$ satifying $0 \leq u \leq \bar{\rho}$. If $\eta$ satisfies 2.4 and $\left(\mathbf{m}_{t}\right)_{t \geq 0}$ satisfies $\left(\mathrm{M}_{4}\right)$, then $u$ is regular in the sense of Remark 3.12$)$

3.3. Existence and uniqueness of bounded nonnegative solution. Combining [2, Theorem 3.1] with a suitable fixed point procedure, we establish the existence of a nonnegative bounded strong solution to the Cauchy problem associated with DCP-structured reaction functionals. Recall that $u$ is called strong solution if it is absolutely continuous in time and satisfies a.e. the equation of $\left(\mathcal{P}_{\mathcal{T}, \eta}\right)$.

Theorem 3.1. Let $F$ be a DCP-structured reaction functional associated with $(r, g, h, q)$, with $\bar{\rho}$ and $\bar{y}$ given by (DCP), $\Phi$ given by (3.1), and assume that $0 \leq \phi \leq a_{0} \bar{\rho}$. Let $\left(\mathbf{m}_{t}\right)_{t>0}$ be a family of vector measure in $\mathbf{M}_{1}^{+}\left(\mathbb{R}, L^{\infty}(\Omega)\right)$ satisfying $\left(\mathrm{M}_{1}\right)$, $\left(\mathrm{M}_{2}\right),\left(\mathrm{M}_{3}\right)$, and $\eta \in C_{c}\left((-\infty, 0], L^{2}(\Omega)\right)$, absolutely continuous, satisfying $\eta(0) \in \overline{\operatorname{dom}(D \Phi)}$, and $0 \leq \eta \leq \bar{\rho}$. Then, for all $T>0$, the time delays reaction-diffusion problem

$$
\left(\mathcal{P}_{\mathcal{T}, \eta}\right)\left\{\begin{array}{l}
\frac{d u}{d t}(t)+D \Phi(u(t))=F(t, u(t), \mathcal{T} u(t)) \text { for a.e. } t \in(0, T) \\
u(t)=\eta(t) \text { for all } t \in(-\infty, 0]
\end{array}\right.
$$

admits a unique solution $u \in C\left((-\infty, T], L^{2}(\Omega)\right)$ satisfying: 
$\left(\mathrm{S}_{1}\right) u(t) \in \operatorname{dom}(D \Phi)$ for a.e. $t \in(0, T)$,

$\left(\mathrm{S}_{2}\right)$ u is almost everywhere derivable in $(0, T)$ and $u^{\prime}(t)=\frac{d u}{d t}(t)$ for a.e. $t \in(0, T)$,

$\left(\mathrm{S}_{3}\right) u(t) \in[0, \bar{y}(T)]$ for all $t \in[0, T]$.

If moreover $F$ is a regular DCP-structured reaction functional, $\eta$ satisfies 2.4 and $\left(\mathbf{m}_{t}\right)_{t>0}$ fulfills condition $\left(\mathrm{M}_{4}\right)$, then u satisfies

$\left(\mathrm{S}_{4}\right) u(t) \in \operatorname{dom}(D \Phi)$ for all $\left.\left.t \in\right] 0, T\right], u$ admits a right derivative $\frac{d u^{+}}{d t}(t)$ at every $\left.\left.t \in\right] 0, T\right][4$ and

$$
\frac{d u^{+}}{d t}(t)+D \Phi(u(t))=F(t, u(t), \mathcal{T} u(t)) .
$$

Proof. Step 1 (local existence). We establish existence of a unique solution of $\left(\mathcal{P}_{\mathcal{T}, \eta}\right)$ satisfying $\left(\mathrm{S}_{1}\right)\left(\mathrm{S}_{4}\right)$ for $T$ small enough. For all $T>0$, set

$$
X_{T}:=\left\{v \in C((-\infty, T], X): 0 \leq v \leq \bar{y}(T) \text { and } v_{\lfloor(-\infty, 0]}=\eta\right\} .
$$

Choose $M$ large enough such that $\operatorname{Support}(\eta) \subset[-M, 0]$. Clearly $X_{T}$ is a closed subset of the Banach space $C([-M, T], X)$ equipped with the uniform norm, Therefore, $X_{T}$ equipped with the distance associated with the uniform norm, is a complete metric space. For each fixed $u$ in $X_{T}$, consider the reaction-diffusion problem with unknown $\Lambda u$ defined by

$$
\left(\mathcal{P}_{\Lambda}\right)\left\{\begin{array}{l}
\frac{d \Lambda u}{d t}(t)+D \Phi(\Lambda u(t))=F(t, \Lambda u(t), \mathcal{T} u(t)) \text { for a.e. } t \in(0, T) \\
\Lambda u(t)=\eta(t) \text { for all } t \in(-\infty, 0],
\end{array}\right.
$$

and first prove that $\left(\mathcal{P}_{\Lambda}\right)$ admits a unique solution $\Lambda u$ satisfying $\left.\left[\mathrm{S}_{1}\right),\left(\mathrm{S}_{2}\right)\right]$ and $\left(\mathrm{S}_{3}\right)$. Note that it suffices to prove existence of $\Lambda u$ in $C\left([0, T], L^{2}(\Omega)\right)$ which solves

$$
\left\{\begin{array}{l}
\frac{d \Lambda u}{d t}(t)+D \Phi(\Lambda u(t))=F(t, \Lambda u(t), \mathcal{T} u(t)) \text { for a.e. } t \in(0, T) \\
\Lambda u(0)=\eta(0)
\end{array}\right.
$$

and to extend $\Lambda u$ by $\eta$ on $(-\infty, 0]$. Since $F$ is a DCP-structured reaction functional, $F$ is associated with $f$ given by $f\left(t, x, \zeta, \zeta^{\prime}\right)=r(t, x) \odot h\left(\zeta^{\prime}\right) \cdot g(\zeta)+q(t, x)$.

Set $r_{\mathcal{T}}(t, x):=r(t, x) \odot h(\mathcal{T} u(t)(x)), f_{\mathcal{T}}(t, x, \zeta):=r_{\mathcal{T}}(t, x) \cdot g(\zeta)+q(t, x)$ and, for $u \in L^{2}(\Omega)$, $F_{\mathcal{T}}(t, u)(x):=f_{\mathcal{T}}(t, x, u(x))$. We have

$$
\begin{aligned}
F(t, \Lambda u(t), \mathcal{T} u(t))(x) & =r(t, x) \odot h(\mathcal{T} u(t)(x)) \cdot g(\Lambda u(t, x))+q(t, x) \\
& =r_{\mathcal{T}}(t, x) \cdot g(\Lambda u(t, x))+q(t, x) \\
& =f_{\mathcal{T}}(t, x, \Lambda u(t, x)):=F_{\mathcal{T}}(t, \Lambda u(t))(x) .
\end{aligned}
$$

The functional $F_{\mathcal{T}}$ is a CP-structured reaction functional associated with $\left(r_{\mathcal{T}}, g, q\right)$ as defined in 2 (See Definition B.1). Condition (CP) holds because, from Lemma 2.1, $0 \leq \mathcal{T} u \leq \bar{y}(T)$, and $f$ satisfies (DCP) Therefore, existence and uniqueness of $\Lambda u$, which fulfills $\left.\left(\mathrm{S}_{1}\right),\left(\mathrm{S}_{2}\right),\left(\mathrm{S}_{3}\right)\right]$ is ensured by [2, Theorem 3.1] (see Theorem B.1).

Assume that $F$ is regular, i.e., $r \in W^{1,1}\left(0, T, L^{2}\left(\Omega, \mathbb{R}^{l}\right)\right), q \in W^{1,1}\left(0, T, L^{2}(\Omega)\right)$ for all $T>0$, that $\eta$ satisfies (2.4), and $\left(\mathbf{m}_{t}\right)_{t>0}$ fulfills condition $\left(\mathrm{M}_{4}\right)$ To show that $\Lambda u$ satisfies $\left(\mathrm{S}_{4}\right)$, according to [2, Theorem 3.1], it remains to prove that $r_{\mathcal{T}}: t \mapsto r_{\mathcal{T}}(t, \cdot)$ is absolutely continuous from $[0, T]$ into $L^{2}(\Omega)$. The claim follows from the absolute continuity of $r$, condition $\left(\mathrm{M}_{4}\right)$ (note that $u \in C_{\eta}\left((-\infty, T], L^{2}(\Omega)\right)$ ),

\footnotetext{
${ }^{4}$ The right derivative at $T$ is the right derivative at $T$ of the extension of $u$ to $[T,+\infty)$ (see Step 3 of the proof)
} 
and the following estimate for $s$ and $t$ in $[0, T]$ :

$$
\begin{aligned}
\left\|r_{\mathcal{T}}(t, \cdot)-r_{\mathcal{T}}(s, \cdot)\right\|_{L^{2}\left(\Omega, \mathbb{R}^{l}\right)}= & \|r(t, \cdot) \odot h(\mathcal{T} u(t))-r(s, \cdot) \odot h(\mathcal{T} u(s))\|_{L^{2}\left(\Omega, \mathbb{R}^{l}\right)} \\
\leq & \|r(t, \cdot) \odot h(\mathcal{T} u(t))-r(s, \cdot) \cdot h(\mathcal{T} u(t))\|_{L^{2}\left(\Omega, \mathbb{R}^{l}\right)} \\
& +\|r(s, \cdot) \odot h(\mathcal{T} u(t))-r(s, \cdot) \odot h(\mathcal{T} u(s))\|_{L^{2}\left(\Omega, \mathbb{R}^{l}\right)} \\
\leq & \|h\|_{L^{\infty}\left([0, \bar{y}(T)], \mathbb{R}^{l}\right)}\|r(t, \cdot)-r(s, \cdot)\|_{L^{2}\left(\Omega, \mathbb{R}^{l}\right)} \\
& +\|r\|_{L^{\infty}\left([0, T] \times \mathbb{R}^{N}, \mathbb{R}^{l}\right)} L_{h, T}\|\mathcal{T} u(t)-\mathcal{T} u(s)\|_{L^{2}(\Omega)} \\
\leq & \|h\|_{L^{\infty}\left([0, \bar{y}(T)], \mathbb{R}^{l}\right)}\|r(t, \cdot)-r(s, \cdot)\|_{L^{2}\left(\Omega, \mathbb{R}^{l}\right)} \\
& +\|r\|_{L^{\infty}\left([0, T] \times \mathbb{R}^{N}, \mathbb{R}^{l}\right)} L_{h, T} \int_{s}^{t} \varphi_{u}(\tau) d \tau,
\end{aligned}
$$

where $L_{h, T}$ denotes the Lipschitz constant of the restriction of $h$ to $[0, \bar{y}(T)]$.

We are going to apply the Banach fixed point theorem to $\Lambda: X_{T} \rightarrow C([-M, T], X)$. Clearly $\Lambda\left(X_{T}\right) \subset$ $X_{T}$. Indeed estimate $0 \leq \Lambda u \leq \bar{y}(T)$ for all $t \in(-\infty, T]$ is due to hypothesis $0 \leq \eta \leq \bar{\rho} \leq \bar{y}(T)$, to $\Lambda u(t)=\eta(t)$ for $t \in(-\infty, 0]$, and to the fact that $\Lambda u$ satisfies $\left[\mathrm{S}_{3}\right)$. To complete the proof, it remains to establish that $\Lambda: X_{T} \rightarrow X_{T}$ is a contraction for $T$ small enough.

Let $(u, v) \in X_{T} \times X_{T}$. Then, for a.e. $t \in(0, T)$ we have

$$
\begin{aligned}
& \frac{d \Lambda u}{d t}(t)+D \Phi(\Lambda u(t))=F(t, \Lambda u(t), \mathcal{T} u(t)), \\
& \frac{d \Lambda v}{d t}(t)+D \Phi(\Lambda v(t))=F(t, \Lambda v(t), \mathcal{T} v(t)) .
\end{aligned}
$$

Subtract these two equalities and take the scalar product with $\Lambda u(t)-\Lambda v(t)$ in $X$. This yields

$$
\begin{aligned}
& \left\langle\frac{d}{d t}(\Lambda u-\Lambda v)(t),(\Lambda u-\Lambda v)(t)\right\rangle+\langle D \Phi(\Lambda u(t))-D \Phi(\Lambda v(t)), \Lambda u(t)-\Lambda v(t)\rangle \\
& =\langle F(t, \Lambda u(t), \mathcal{T} u(t))-F(t, \Lambda v(t), \mathcal{T} v(t)), \Lambda u(t)-\Lambda v(t)\rangle .
\end{aligned}
$$

Then, using the fact that $D \Phi$ is a monotone operator, we infer that for a.e. $t \in(0, T)$

$$
\frac{1}{2} \frac{d}{d t}\|(\Lambda u-\Lambda v)(t)\|_{X}^{2} \leq\langle F(t, \Lambda u(t), \mathcal{T} u(t))-F(t, \Lambda v(t), \mathcal{T} v(t)), \Lambda u(t)-\Lambda v(t)\rangle .
$$

Thus, for a.e. $t \in(0, T)$,

$$
\begin{aligned}
\frac{d}{d t}\|\Lambda u(t)-\Lambda v(t)\|_{X}^{2} & \leq 2\|F(t, \Lambda u(t), \mathcal{T} u(t))-F(t, \Lambda v(t), \mathcal{T} v(t))\|_{X}\|\Lambda u(t)-\Lambda v(t)\|_{X} \\
& \leq\|F(t, \Lambda u(t), \mathcal{T} u(t))-F(t, \Lambda v(t), \mathcal{T} v(t))\|_{X}^{2}+\|\Lambda u(t)-\Lambda v(t)\|_{X}^{2} .
\end{aligned}
$$

According to the structure of the DCP-structured reaction functional $F$, from $(2.3)$ and the fact that $u=v$ in $(-\infty, 0]$, we have

$$
\begin{aligned}
& \|F(t, \Lambda u(t), \mathcal{T} u(t))-F(t, \Lambda v(t), \mathcal{T} v(t))\|_{X}^{2} \\
& \leq C(T, g, h)\|\mathcal{T} u(t)-\mathcal{T} v(t)\|_{X}^{2}+C^{\prime}(T, g, h)\|\Lambda u(t)-\Lambda v(t)\|_{X}^{2} \\
& \leq 4 T C(T, g, h)\|u(t)-v(t)\|_{X}+C^{\prime}(T, g, h)\|\Lambda u(t)-\Lambda v(t)\|_{X}^{2}
\end{aligned}
$$

where

$$
\begin{aligned}
& C(T, g, h)=2 \sup _{\zeta \in[0, \bar{y}(T)]}|g(\zeta)|^{2}\|r\|_{L^{\infty}\left(\mathbb{R}^{N}, \mathbb{R}^{l}\right)}^{2} L_{h, T}(T)^{2}, \\
& C^{\prime}(T, g, h)=2 \sup _{\zeta^{\prime} \in[0, \bar{y}(T)]}\left|h\left(\zeta^{\prime}\right)\right|^{2}\|r\|_{L^{\infty}\left(\mathbb{R}^{N}, \mathbb{R}^{l}\right)}^{2} L_{g, T}(T)^{2}
\end{aligned}
$$

(recall that $L_{g, T}$ and $L_{h, T}$ denote the Lipschitz constants of the restrictions of $g$ and $h$ on $[0, \bar{y}(T)]$ respectively). Combining (3.6) and (3.7) we infer that for a.e. $t \in(0, T)$

$$
\frac{d}{d t}\|\Lambda u(t)-\Lambda v(t)\|_{X}^{2} \leq 4 T C(T, g, h)\|u(t)-v(t)\|_{X}^{2}+\left(1+C^{\prime}(T, g, h)\right)\|\Lambda u(t)-\Lambda v(t)\|_{X}^{2} .
$$

By integrating this inequality over $(0, s)$ for $s \in(0, T)$ and noticing that $\Lambda u(0)=\Lambda v(0)=\eta(0)$, we obtain

$$
\|\Lambda u(s)-\Lambda v(s)\|_{X}^{2} \leq 4 T^{2} C(T, g, h)\|u-v\|_{C((-\infty, T], X)}^{2}+\left(1+C^{\prime}(T, g, h)\right) \int_{0}^{s}\|\Lambda u(t)-\Lambda v(t)\|_{X}^{2} d t
$$


from which, according to Grönwall's lemma, we deduce that for all $s \in(0, T)$,

$$
\|\Lambda u(s)-\Lambda v(s)\|_{X}^{2} \leq 4 T^{2} C(T, g, h)\|u-v\|_{C((-\infty, T], X)}^{2} \exp \left(\left(1+C^{\prime}(T, g, h)\right) T\right) .
$$

Since $\Lambda u=\Lambda v=\eta$ on $(-\infty, 0]$, this inequality holds for all $s \in(-\infty, T]$ so that

$$
\|\Lambda u-\Lambda v\|_{C((-\infty, T], X)} \leq K(T)\|u-v\|_{C((-\infty, T], X)}
$$

where $K(T):=2 T(C(T, g, h))^{\frac{1}{2}} \exp \left(\left(1+C^{\prime}(T, g, h)\right) \frac{T}{2}\right)$. We claim that $\lim _{T \rightarrow 0} K(T)=0$. The proof will be completed by choosing $T^{*}>0$ small enough so that $K\left(T^{*}\right)<1$. For this, note that the maps $T \mapsto \bar{y}(T), T \mapsto L_{g, T}$, and $T \mapsto L_{h, T}$ are non decreasing so that $C(T, g, h)$ and $C(T, g, h)$ decrease to finite limits when $T$ decreases to 0 .

Step 2 (uniqueness). For $T^{*}$ obtained above, we establish the uniqueness of the solution of $\left(\mathcal{P}_{\mathcal{T}, \eta}\right)$. Let $u$ and $v$ be two solutions, then, by taking $u$ for $\Lambda u$ and $v$ for $\Lambda v$ in (3.8), we infer that for a.e. $t \in\left(0, T^{*}\right)$

$$
\frac{d}{d t}\|u(t)-v(t)\|_{X}^{2} \leq\left(1+\left(C\left(T^{*}, g, h\right)+C^{\prime}\left(T^{*}, g, h\right)\right)\right)\|u(t)-v(t)\|_{X}^{2} .
$$

Applying Grönwall's Lemma after integrating over $(0, s)$, we obtain

$$
\|u(t)-v(t)\|_{X}^{2} \leq\|u(0)-v(0)\|_{X}^{2} \exp \left(\left(1+\left(C\left(T^{*}, g, h\right)+C^{\prime}\left(T^{*}, g, h\right)\right)\right) T^{*}\right)
$$

and the claim follows since $u(0)=v(0)=\eta(0)$.

Step 3 (existence of a global solution). By Step 1 consider $T^{*}>0$ such that $\left(\mathcal{P}_{\mathcal{T}, \eta}\right)$ admits a unique solution in $C\left(\left[0, T^{*}\right], X\right)$. Under the initial condition $u(0)=\eta(0) \in \overline{\operatorname{dom}(D \Phi)}$ we are not assured that the derivative $\frac{d u}{d t}$ of the solution belongs to $L^{2}\left(0, T^{*}, X\right)$. Nevertheless $\sqrt{t} \frac{d u}{d t} \in L^{2}\left(0, T^{*}, X\right)$ (see [4. Theorem 17.2.5] or [6. Theorem 3.6]). Hence, for $0<\delta<T^{*}, \frac{d u}{d t}$ belongs to $L^{2}\left(\delta, T^{*}, X\right)$. Set

$$
E:=\left\{T>\delta: \exists u \in C((-\infty, T], X), u \text { solution of }\left(\mathcal{P}_{\mathcal{T}, \eta}\right)\right\} .
$$

Since $T^{*} \in E$, we have $E \neq \emptyset$. Set $T_{\text {Max }}:=\sup E$ in $\overline{\mathbb{R}}_{+}$. In the following we denote by $u$ the maximal solution of $\left(\mathcal{P}_{\mathcal{T}, \eta}\right)$ in $C\left(\left[0, T_{M a x}\right), X\right)$ and argue by contradiction by assuming that $T_{\text {Max }}<+\infty$.

a) We first show that $\lim _{t \rightarrow T_{M a x}} u(t)$ exists in $X$. Let $T \in E$, then for a.e. $t \in(0, T)$ we have

$$
\left\langle\frac{d u}{d t}(t), \frac{d u}{d t}(t)\right\rangle+\left\langle D \Phi u(t), \frac{d u}{d t}(t)\right\rangle=\left\langle F(t, u(t), \mathcal{T} u(t)), \frac{d u}{d t}(t)\right\rangle .
$$

We know that for a.e. $t \in(\delta, T), \frac{d}{d t} \Phi(u(t))=\left\langle D \Phi u(t), \frac{d u}{d t}(t)\right\rangle$ (see [4, Proposition 17.2.5]) so that by integration over $(\delta, T)$ we obtain

$$
\int_{\delta}^{T}\left\|\frac{d u}{d t}(t)\right\|_{X}^{2} d t+\Phi(u(t))-\Phi(u(\delta)) \leq\left(\int_{0}^{T}\|F(t, u(t), \mathcal{T} u(t))\|_{X}^{2} d t\right)^{\frac{1}{2}}\left(\int_{\delta}^{T}\left\|\frac{d u}{d t}(t)\right\|_{X}^{2} d t\right)^{\frac{1}{2}}
$$

Note that for all $T \in E$, we have $[0, \bar{y}(T)] \subset\left[0, \bar{y}\left(T_{M a x}\right)\right]$. Thus, from estimates $0 \leq u \leq \bar{y}(T)$ and $0 \leq \mathcal{T} u \leq \bar{y}(T)$, according to the structure of the DCP-structured reaction fuctionnal $F$, there exists a constant

$$
C\left(\|r\|_{L^{\infty}\left(\mathbb{R}^{N}, \mathbb{R}^{l}\right)},\|g\|_{L^{\infty}\left(\left[0, \bar{y}\left(T_{M a x}\right)\right], \mathbb{R}^{l}\right)},\|h\|_{L^{\infty}\left(\left[0, \bar{y}\left(T_{M a x}\right)\right], \mathbb{R}^{l}\right)}\right)
$$

(that we write $C$ in short) such that

$$
\|F(t, u(t), \mathcal{T} u(t))\|_{X}^{2} \leq 2 C^{2} \mathcal{L}_{N}(\Omega)+2\|q(t, \cdot)\|_{X}^{2} .
$$

Therefore, since $\inf \Phi>-\infty$, and $q \in L^{2}\left(0, T_{M a x}, L^{2}(\Omega)\right)$, 3.9. yields

$$
\int_{\delta}^{T}\left\|\frac{d u}{d t}(t)\right\|_{X}^{2} d t \leq C\left(1+\left(\int_{\delta}^{T}\left\|\frac{d u}{d t}(t)\right\|_{X}^{2} d t\right)^{\frac{1}{2}}\right)
$$

where the new constant $C$ does not depend on $T$. We infer that

$$
\int_{\delta}^{T_{\text {Max }}}\left\|\frac{d u}{d t}(t)\right\|_{X}^{2} d t=\sup _{T \in E} \int_{\delta}^{T}\left\|\frac{d u}{d t}(t)\right\|_{X}^{2} d t<+\infty .
$$


From 3.10 we deduce that $u:\left[\delta, T_{\text {Max }}\right) \rightarrow X$ is uniformly continuous. Indeed, for $s<t$ in $\left[\delta, T_{\text {Max }}\right)$ we have

$$
\|u(t)-u(s)\|_{X} \leq \int_{s}^{t}\left\|\frac{d u}{d \tau}(\tau)\right\|_{X}^{2} d \tau \leq(t-s)^{\frac{1}{2}}\left(\int_{\delta}^{T_{M a x}}\left\|\frac{d u}{d \tau}(t)\right\|_{X}^{2} d t\right)^{\frac{1}{2}} .
$$

Since $X$ is a Banach space, according to the continuous extension principle, $u$ possesses a unique continuous extension $\bar{u}$ in $\left[\delta, T_{\text {Max }}\right]$, i.e., $\lim _{t \rightarrow T_{\text {Max }}} u(t)=\bar{u}\left(T_{\text {Max }}\right)$, which proves the claim.

b) Contradiction: consider the distributed delays reaction-diffusion problem

$$
\left(\mathcal{P}_{\mathcal{T}, \eta^{\prime}}\right)\left\{\begin{array}{l}
\frac{d v}{d t}(t)+D \Phi(v(t))=F(t, v(t), \mathcal{T} v(t)) \text { for a.e. } t \in(0, T) \\
v(t)=\eta^{\prime}(t) \text { for } t \in(-\infty, 0],
\end{array}\right.
$$

where $\eta^{\prime}$ is defined by $\eta^{\prime}(t)=\bar{u}\left(t+T_{M a x}\right)$. It is easily seen that $\eta^{\prime} \in C_{c}\left((-\infty, 0], L^{2}(\Omega)\right)$ and satisfies (2.4). Note that $\eta^{\prime}(0)=\bar{u}\left(T_{M a x}\right) \in \overline{\operatorname{dom}(D \Phi)}$. Indeed, choose $t_{n} \rightarrow T_{\text {Max }}$ with $t_{n}$ outside the negligible set in which $u(t) \notin \operatorname{dom}(D \Phi)$ and use the fact that $\bar{u}\left(T_{M a x}\right)=\lim _{t \rightarrow T_{M a x}} u(t)$. Moreover $0 \leq \eta^{\prime} \leq \bar{\rho}^{\prime}$ where $\bar{\rho}^{\prime}=\bar{y}\left(T_{\text {Max }}\right)$. Then according to Step 1, there exists $T^{* *}>0$ small enough such that $\left(\mathcal{P}_{\mathcal{T}, \eta}^{\prime}\right)$ possesses a solution $v \in C\left(\left[0, T^{* *}\right], X\right)$. Set

$$
\widetilde{u}(t)=\left\{\begin{array}{l}
u(t) \text { if } t \in\left(-\infty, T_{M a x}\right] \\
v\left(t-T_{\text {Max }}\right) \text { if } t \in\left[T_{\text {Max }}, T_{\text {Max }}+T^{* *}\right] .
\end{array}\right.
$$

Then $\widetilde{u} \in C\left(\left[0, T_{\text {Max }}+T^{* *}\right], X\right)$ is a solution of $\left(\mathcal{P}_{\mathcal{T}, \eta}\right)$, a contradiction with the maximality of $T_{\text {Max }}$.

By an easy adaptation of the proof above, we establish the same result in the context of the convention above, i.e. when $a_{0}=0$ on $\Omega \backslash \Gamma$, is extended by $+\infty$ on $\Gamma$, and $\phi=0$. More precisely,

Theorem 3.2. Let $F$ be a DCP-structured reaction functional associated with $(r, g, h, q)$, with $\bar{\rho}$ and $\bar{y}$ given by (DCP), and $\Phi$ given by

$$
\Phi(u)= \begin{cases}\int_{\Omega} W(x, \nabla u(x)) d x & \text { if } u \in H_{\Gamma}^{1}(\Omega) \\ +\infty & \text { otherwise. }\end{cases}
$$

Let $T$ be any positive numbers, and $\eta$ in $C_{c}\left((-\infty, 0], L^{2}(\Omega)\right)$ satisfying $\eta(0) \in \overline{\operatorname{dom}(D \Phi)}$, and $0 \leq \eta \leq \bar{\rho}$. Then the time delays reaction-diffusion problem

$$
\left(\mathcal{P}_{\mathcal{T}, \eta}\right)\left\{\begin{array}{l}
\frac{d u}{d t}(t)+D \Phi(u(t))=F(t, u(t), \mathcal{T} u(t)) \text { for a.e. } t \in(0, T) \\
u(t)=\eta(t) \text { for all } t \in(-\infty, 0]
\end{array}\right.
$$

admits a unique solution $u \in C\left((-\infty, T], L^{2}(\Omega)\right)$ satisfying $\left(\mathrm{S}_{1}\right),\left(\mathrm{S}_{2}\right)$ and $\left(\mathrm{S}_{3}\right)$, If moreover $r$ belongs to $W^{1,1}\left(0, T, L^{2}\left(\Omega, \mathbb{R}^{l}\right)\right)$, q belongs to $W^{1,1}\left(0, T, L^{2}(\Omega)\right)$, $\eta$ satisfies $(2.4)$ and $\left(\mathbf{m}_{t}\right)_{t>0}$ fulfills condition $\left(\mathrm{M}_{4}\right)$, then $u$ satisfies $\left(\mathrm{S}_{4}\right)$.

Remark 3.2. In the case of single time delay reaction-diffusion problems, for establishing existence and uniqueness for $\left(\mathcal{P}_{\mathcal{T}, \eta}\right)$ we could develop the following alternative constructive proof: $\left(\mathcal{P}_{\mathcal{T}, \eta}\right)$ is equivalent to the finite recursive sequence $\left(\mathcal{P}_{i}\right), i=1 \ldots\left[\frac{T}{\tau}\right]+1$ of reaction-diffusion Cauchy problem of the form

$$
\left(\mathcal{P}_{i}\right)\left\{\begin{array}{l}
\frac{d u^{i}}{d t}(t)+D \Phi\left(u^{i}(t)\right)=F_{i}\left(t, u^{i}(t)\right) \text { for a.e. } t \in((i-1) \tau, i \tau) \\
u^{i}((i-1) \tau)=u^{i-1}((i-1) \tau)
\end{array}\right.
$$

where $u^{0}:=\eta$, and $F_{i}\left(t, u^{i}(t)\right):=F\left(t, u^{i}(t), u^{i-1}(t)\right)$ is clearly a CP-structured reaction functional. By applying [2, Theorem 3.1], we see that there exists a unique solution $u^{i}$ of $\left(\mathcal{P}_{i}\right)$. The function $u$ defined by $u(t)=u^{i}(t)$ for $t \in[(i-1) \tau, i \tau]$ is a solution of $\left(\mathcal{P}_{\mathcal{T}, \eta}\right)$. Uniqueness is a consequence of the following remark: if $u$ solves $\left(\mathcal{P}_{\tau, \eta}\right)$, then the restriction of $u$ in $[(i-1) \tau, i \tau]$ is the unique solution of $\left(\mathcal{P}_{i}\right)$. 


\section{General CONVERGEnCE TheOREMS FOR A ClASS OF DELAYS NONLINEAR REACTION-DIFFUSION} PROBLEMS

Consider a sequence $\left(\Phi_{n}\right)_{n \in \mathbb{N}}$ of functionals of the calculus of variations $\Phi_{n}: L^{2}(\Omega) \rightarrow \mathbb{R} \cup\{+\infty\}$ defined by

$$
\Phi_{n}(u)=\left\{\begin{array}{cc}
\int_{\Omega} W_{n}(x, \nabla u(x)) d x+\frac{1}{2} \int_{\partial \Omega} a_{0, n} u^{2} d \mathcal{H}_{N-1}-\int_{\partial \Omega} h_{n} u d \mathcal{H}_{N-1} & \text { if } u \in H^{1}(\Omega) \\
+\infty & \text { otherwise }
\end{array}\right.
$$

where $h_{n} \in L_{\mathcal{H}_{N-1}}^{2}(\partial \Omega), a_{0, n} \in L_{\mathcal{H}_{N-1}}^{\infty}(\partial \Omega), a_{0, n} \geq 0 \mathcal{H}_{N-1}$-a.e. in $\partial \Omega, a_{0, n} \geq \sigma_{n}$ on $\Gamma \subset \partial \Omega$ with $\mathcal{H}_{N-1}(\Gamma)>0$ for some $\sigma_{n}>0$, and $W_{n}: \mathbb{R}^{N} \times \mathbb{R}^{N} \rightarrow \mathbb{R}$ is a measurable function satisfying the following conditions:

$\left(D_{1, n}\right)$ there exist $\left\{\alpha_{n}\right\}_{n \in \mathbb{N}} \subset \mathbb{R}_{+}^{*}$ and $\left\{\beta_{n}\right\}_{n \in \mathbb{N}} \subset \mathbb{R}_{+}^{*}$ such that for a.e. $x \in \mathbb{R}^{N}$, all $\xi \in \mathbb{R}^{N}$, and all $n \in \mathbb{N}$

$$
\alpha_{n}|\xi|^{2} \leq W_{n}(x, \xi) \leq \beta_{n}\left(1+|\xi|^{2}\right),
$$

$\left(\mathrm{D}_{2, n}\right)$ for a.e. $x \in \mathbb{R}^{N}$ and every $n \in \mathbb{N}$, the function $W_{n}(x, \cdot)$ is convex and differentiable with $D_{\xi} W_{n}(x, 0)=0$ a.e. in $\mathbb{R}^{N}$,

$\left(\mathrm{D}_{3, n}\right)\left(W_{n}\right)_{n \in \mathbb{N}}$ is uniformly strongly convex, i.e., for some $\gamma>0$ it holds for all $\xi \in \mathbb{R}^{N}$,

$$
\inf _{n \in \mathbb{N}} \inf _{x \in \mathbb{R}^{N}} D_{\xi} W_{n}(x, \xi) \cdot \xi \geq \gamma|\xi|^{2} .
$$

From now on, we fix $T>0$, and we assume the following Structural Conditions.

(SC1) We are given a sequence $\left(F_{n}\right)_{n \in \mathbb{N}}$ of regular DCP-structured reaction functionals, each $F_{n}$ is associated with $\left(r_{n}, g_{n}, h_{n}, q_{n}\right)$, i.e., $F_{n}(t, u, v)(x)=f_{n}(t, x, u(x), v(x))$ for all $t \in[0, T]$, a.e. $x \in \Omega$, and all $(u, v) \in L^{2}(\Omega)^{2}$, where

$$
\forall\left(t, x, \zeta, \zeta^{\prime}\right) \in[0,+\infty) \times \mathbb{R}^{N} \times \mathbb{R} \times \mathbb{R} \quad f_{n}\left(t, x, \zeta, \zeta^{\prime}\right)=r_{n}(t, x) \odot h_{n}\left(\zeta^{\prime}\right) \cdot g_{n}(\zeta)+q_{n}(t, x)
$$

For all $n \in \mathbb{N}, h_{n}$ and $g_{n}$ are assumed to be locally Lipschitz functions, uniformly with respect to $n$, i.e., for all interval $I \subset \mathbb{R}$, there exist $L_{I} \geq 0$ and $L_{I}^{\prime} \geq 0$ such that

$$
\forall\left(\zeta, \zeta^{\prime}\right) \in \mathbb{R}^{2} \quad \sup _{n \in \mathbb{N}}\left|g_{n}(\zeta)-g_{n}\left(\zeta^{\prime}\right)\right| \leq L_{I}\left|\zeta-\zeta^{\prime}\right| \quad \text { and } \quad \sup _{n \in \mathbb{N}}\left|h_{n}(\zeta)-h_{n}\left(\zeta^{\prime}\right)\right| \leq L_{I}^{\prime}\left|\zeta-\zeta^{\prime}\right| .
$$

We assume that the absolute continuity of $r_{n}$ and $q_{n}$ holds uniformly with respect to $n$, i.e.,

$$
\sup _{n \in \mathbb{N}} \int_{0}^{T}\left\|\frac{d r_{n}}{d t}(t, \cdot)\right\|_{L^{2}\left(\Omega, \mathbb{R}^{l}\right)} d t<+\infty \quad \text { and } \quad \sup _{n \in \mathbb{N}} \int_{0}^{T}\left\|\frac{d q_{n}}{d t}(t, \cdot)\right\|_{L^{2}(\Omega)} d t<+\infty .
$$

(SC2) We are given a sequence $\left(\eta_{n}\right)_{n \in \mathbb{N}}$ of history functions in $C_{c}\left((-\infty, 0], L^{2}(\Omega)\right)$ satisfying $\eta_{n}(0) \in$ $H^{1}(\Omega), 0 \leq \eta_{n} \leq \bar{\rho}_{n}$, and

$$
\sup _{n \in \mathbb{N}} \int_{-\infty}^{0}\left\|\frac{d \eta_{n}}{d t}(t, \cdot)\right\|_{L^{2}(\Omega)} d t<+\infty
$$

where $\bar{\rho}_{n}$ is given by the condition (DCP) fulfilled by each $F_{n}$.

(SC3) We are given a sequence $\left(\mathbf{m}_{t}^{n}\right)_{t>0, n \in \mathbb{N}}$ of vector measures in $\mathbf{M}_{1}^{+}\left(\mathbb{R}, L^{\infty}(\Omega)\right)$ such that each $\left(\mathbf{m}_{t}^{n}\right)_{t \geq 0}$ satisfies $\left(\mathrm{M}_{1}\right),\left(\mathrm{M}_{2}\right)$ and $\left(\mathrm{M}_{3}\right)$ The sequence of time-delays operator $\left(\mathcal{T}_{n}\right)_{n \in \mathbb{N}}$ associated with $\left(\mathbf{m}_{t}^{n}\right)_{t \geq 0, n \in \mathbb{N}}$, is defined for each $v$ in $C_{c}\left((-\infty, T], L^{2}(\Omega)\right)$, by

$$
\mathcal{T}_{n} v(t)=\int_{-\infty}^{t} v(s) d \mathbf{m}_{t}^{n}(s)
$$

(see Definition 2.1.

Furthermore we assume that each family of vector measure $\left(\mathbf{m}_{t}^{n}\right)_{t>0}$ fulfills condition $\left(\mathrm{M}_{4}^{\prime}\right)$ More precisely, there exists a sequence of probability measure $\left(\mu_{n}\right)_{n \in \mathbb{N}}$ in $\mathcal{P}_{+}(\mathbb{R})$, such that for all 
$v$ in $C_{\eta_{n}}\left((-\infty, T], L^{2}(\Omega)\right)$

$\forall(s, t) \in[0, T]^{2} \quad s<t \Longrightarrow\left\|\mathcal{T}_{n} v(t)-\mathcal{T}_{n} v(s)\right\|_{L^{2}(\Omega)} \leq \int_{s}^{t}\left(\left\|\frac{d v}{d \sigma}(\cdot)\right\|_{L^{2}(\Omega)} \star \mu_{n}\right)(\sigma) d \sigma$.

(SC4) Finally, we assume that

$$
\bar{\rho}:=\sup _{n \in \mathbb{N}} \bar{y}_{n}(T)<+\infty
$$

and

$$
\forall n \in \mathbb{N} \quad 0 \leq \phi_{n} \leq a_{0, n} \bar{\rho}_{n} \text { on } \partial \Omega
$$

where $\bar{y}_{n}$ is given by the condition (DCP) fulfilled by each $F_{n}$. Note that when considering the case

$$
a_{0, n}(x)= \begin{cases}0 & \text { if } x \in \partial \Omega \backslash \Gamma, \\ +\infty & \text { if } x \in \Gamma,\end{cases}
$$

and $\phi_{n}=0$, for all $n \in \mathbb{N}$, then condition 4.7) is automatically satisfied.

4.1. Stability at the limit. According to the structure of the reaction functional $F_{n}$, the time delays term, and a suitable notion of weak convergence relating to $\left(\mathbf{m}_{t}^{n}\right)_{t>0}$, in theorem below we show that the limit of the time delays problems $\left(\mathcal{P}_{n}\right)$ associated with above datas, keeps the same structure.

Theorem 4.1 (No mixing effect). Assume that the sequence $\left(W_{n}\right)_{n \in \mathbb{N}}$ satisfies conditions $\left(\mathrm{D}_{1, n}\right),\left(\mathrm{D}_{2, n}\right),\left(\mathrm{D}_{3, n}\right)$, and that the sequence of DCP-structured reaction functionals $\left(F_{n}\right)_{n \in \mathbb{N}}$ satisfies $(\mathrm{SC} 1)$, $(\mathrm{SC} 2)$, $\left.(\mathrm{SC} 3)\right]$ and $[\mathrm{SC} 4)$, Let $u_{n}$ be the unique solution of the time delays problem

$$
\left(\mathcal{P}_{n}\right)\left\{\begin{array}{l}
\frac{d u_{n}}{d t}(t)+D \Phi_{n}\left(u_{n}(t)\right)=F_{n}\left(t, u_{n}(t), \mathcal{T}_{n} u_{n}(t)\right) \text { for a.e. } t \in(0, T), \\
u_{n}(t)=\eta_{n}(t) \text { for all } t \in(-\infty, 0] .
\end{array}\right.
$$

Assume that

$\left(\mathrm{H}_{1}\right) \Phi_{n} \stackrel{M}{\rightarrow} \Phi$ and $\sup _{n \in \mathbb{N}}\left\|\phi_{n}\right\|_{L_{\mathcal{H}_{N-1}}^{2}(\partial \Omega)}<+\infty ;$

$\left(\mathrm{H}_{2}\right) \sup _{n \in \mathbb{N}} \Phi_{n}\left(\eta_{n}(0)\right)<+\infty ;$

$\left(\mathrm{H}_{3}\right)$ there exists $\eta \in C_{c}\left((-\infty, 0], L^{2}(\Omega)\right)$ such that $\eta_{n} \rightarrow \eta$ in $C\left((-\infty, 0], L^{2}(\Omega)\right)$;

$\left(\mathrm{H}_{4}\right)$ there exists a family of $L^{\infty}(\Omega)$-valued measures $\left(\mathbf{m}_{t}\right)_{t>0}$ with finite variation, such that for all $\left.t \in[0, T], \mathbf{m}_{t}^{n} L_{(-\infty, t]} \rightarrow \mathbf{m}_{t} L_{(-\infty, t}\right]^{5}$ for the weak convergence of measures, in $L^{2}(\Omega)$ strong, i.e., for all $\psi \in C_{c}\left(\mathbb{R}, L^{2}(\Omega)\right), \lim _{n \rightarrow \infty} \int_{\mathbb{R}} \mathbb{1}_{(-\infty, t]} \psi d \mathbf{m}_{t}^{n}=\int_{\mathbb{R}} \mathbb{1}_{(-\infty, t]} \psi d \mathbf{m}_{t}$ strongly in $L^{2}(\Omega)$;

$\left(\mathrm{H}_{5}\right) g_{n}$ and $h_{n}$ pointwise converge to $g$ and $h$ respectively;

$\left(\mathrm{H}_{6}\right) \bar{r}:=\sup _{n \in \mathbb{N}}\left\|r_{n}\right\|_{L^{\infty}\left([0, T] \times \mathbb{R}^{N}, \mathbb{R}^{l}\right)}<+\infty$ and $r_{n} \rightarrow r$ in $L^{2}\left(0, T, L^{2}\left(\Omega, \mathbb{R}^{l}\right)\right)$ where $r \in L^{\infty}\left([0, T] \times \mathbb{R}^{N}, \mathbb{R}^{l}\right) ;$

$\left(\mathrm{H}_{7}\right)$ for all $t \in[0, T], \sup _{n \in \mathbb{N}}\left\|q_{n}(t, \cdot)\right\|_{L^{2}(\Omega)}<+\infty$ and $q_{n} \rightarrow q$ in $L^{2}\left(0, T, L^{2}(\Omega)\right)$.

Then $u_{n}$ uniformly converges in $C\left([0, T], L^{2}(\Omega)\right)$ to some function $u$ whose extension by $\eta$ in $(-\infty, 0]$, is the unique solution of the time delays problem

$$
(\mathcal{P})\left\{\begin{array}{l}
\frac{d u}{d t}(t)+\partial \Phi(u(t)) \ni F(t, u(t), \mathcal{T} u(t)) \text { for a.e. } t \in(0, T) \\
u(t)=\eta(t), 0 \leq \eta \leq \bar{\rho}:=\sup _{n \in \mathbb{N}} \bar{y}_{n}(T) \text { for all } t \in(-\infty, 0], \eta(0) \in \operatorname{dom}(\Phi) .
\end{array}\right.
$$

\footnotetext{
${ }^{5}$ For any Borel vector measure $\mu$, and Borel set $B$, we denote by $\mu\left\lfloor_{B}\right.$ the restriction of $\mu$ to the $\sigma$-algebra restriction of $\mathcal{B}(\mathbb{R})$ to $B$
} 
The reaction functional $F:[0,+\infty) \times L^{2}(\Omega) \times L^{2}(\Omega) \rightarrow \mathbb{R}^{\Omega}$ is defined for all $t \in[0, T]$, all $(u, v) \in L^{2}(\Omega)^{2}$ and for a.e. $x \in \Omega$, by

$$
\begin{aligned}
& F(t, u, v)(x)=f(t, x, u(x), v(x)), \\
& f\left(t, x, \zeta, \zeta^{\prime}\right)=r(t, x) \odot h\left(\zeta^{\prime}\right) \cdot g(\zeta)+q(t, x) .
\end{aligned}
$$

The operator $\mathcal{T}: C_{c}\left((-\infty, T], L^{2}(\Omega)\right) \rightarrow L^{2}\left(0, T, L^{2}(\Omega)\right)$ is defined by

$$
\forall t \in[0, T] \quad \mathcal{T} u(t)=\int_{-\infty}^{t} u(s) d \mathbf{m}_{t}(s) .
$$

Moreover $\frac{d u_{n}}{d t} \rightarrow \frac{d u}{d t}$ weakly in $L^{2}\left(0, T, L^{2}(\Omega)\right)$, and $0 \leq u \leq \bar{\rho}:=\sup _{n \in \mathbb{N}} \bar{y}_{n}(T)$.

Proof. We follow the outlines of the proof of [2, Theorem 4.1]. The additional difficulty is the presence of the delay term in the reaction functional. In the statement of Theorem 4.1. from (SC2) we assume that $u_{n}^{0}=\eta_{n}(0) \in H^{1}(\Omega)=\operatorname{dom}\left(\Phi_{n}\right)$. But $\operatorname{dom}\left(\Phi_{n}\right) \subset \overline{\operatorname{dom}\left(\Phi_{n}\right)}=\overline{\operatorname{dom}\left(D \Phi_{n}\right)}$ (for this last point we refer to [4, Lemma 17.4.1]), hence $u_{n}^{0} \in \overline{\operatorname{dom}\left(D \Phi_{n}\right)}$. Therefore, according to Theorem 3.1, $\left(\mathcal{P}_{n}\right)$ possesses a unique solution $u_{n}$ which satisfies $\left(\mathrm{S}_{2}\right)\left(\mathrm{S}_{4}\right)$. Note that, from Lemma 2.1. we have $0 \leq \mathcal{T}_{n} u_{n} \leq \bar{y}_{n}(T)$.

Step 1. We establish

$$
\begin{aligned}
& 0 \leq \eta \leq \bar{\rho} ; \\
& \sup _{n \in \mathbb{N}}\left\|u_{n}\right\|_{C(0, T, X)} \leq \mathcal{L}_{N}(\Omega)^{\frac{1}{2}} \bar{\rho} ; \\
& \bar{g}:=\sup _{(\zeta, n) \in[0, \bar{\rho}] \times \mathbb{N}}\left|g_{n}(\zeta)\right|<+\infty, \bar{h}:=\sup _{(\zeta, n) \in[0, \bar{\rho}] \times \mathbb{N}}\left|h_{n}(\zeta)\right|<+\infty ; \\
& \sup _{n \in \mathbb{N}}\left\|\frac{d u_{n}}{d t}\right\|_{L^{2}(0, T, X)}<+\infty .
\end{aligned}
$$

(recall that $\bar{\rho}:=\sup _{n \in \mathbb{N}} \bar{y}_{n}(T)$ which belong to $\mathbb{R}$ from 4.6 ). Inequality 4.8 follows directly from $0 \leq \eta_{n} \leq \bar{\rho}_{n} \leq \bar{y}_{n}(T) \leq \bar{\rho}$ given by $(\mathrm{SC} 2)$, and $\left(\mathrm{H}_{3}\right)$ Inequality 4.9 follows from $0 \leq u_{n} \leq \bar{y}_{n}(T) \leq \bar{\rho}$. We easily deduce 4.10 from $(4.2)$, hypothesis $\left(\mathrm{H}_{5}\right)$ and estimate $\left|g_{n}(\zeta)\right| \leq\left|g_{n}(0)\right|+L_{[0, \bar{\rho}]}|\zeta|$, idem for $h_{n}$.

Let us establish 4.11). In what follows the letter $C$ denotes various constants which can vary from line to line. By using the structure of the DCP-structured reaction functional $F_{n}$, and from 4.10 and hypothesis $\left(\mathrm{H}_{6}\right)$, we infer that

$$
\begin{aligned}
\left\|F_{n}\left(t, u_{n}(t), \mathcal{T}_{n} u_{n}(t)\right)\right\|_{X}^{2} & \leq 2 \mathcal{L}(\Omega)\left\|r_{n}\right\|_{\infty}^{2} \bar{g}^{2} \bar{h}^{2}+2\left\|q_{n}(t, \cdot)\right\|_{X}^{2} \\
& \leq C\left(1+\left\|q_{n}(t, \cdot)\right\|_{X}^{2}\right) .
\end{aligned}
$$

Thus, according to hypothesis $\left(\mathrm{H}_{7}\right)$, we deduce

$$
\sup _{n \in \mathbb{N}} \int_{0}^{T}\left\|F_{n}\left(t, u_{n}(t), \mathcal{T}_{n} u_{n}(t)\right)\right\|_{X}^{2} d t<+\infty .
$$

On the other hand, since $u_{n}$ solves $\left(\mathcal{P}_{n}\right)$ we have for a.e. $t \in(0, T)$,

$$
\left\|\frac{d u_{n}}{d t}(t)\right\|^{2}+\left\langle D \Phi_{n}\left(u_{n}(t)\right), \frac{d u_{n}}{d t}(t)\right\rangle=\left\langle F_{n}\left(t, u_{n}(t), \mathcal{T}_{n} u_{n}(t)\right), \frac{d u_{n}}{d t}(t)\right\rangle
$$

and, by integrating this equality over $(0, T)$,

$$
\int_{0}^{T}\left\|\frac{d u_{n}}{d t}(t)\right\|^{2} d t+\int_{0}^{T}\left\langle D \Phi_{n}\left(u_{n}(t)\right), \frac{d u_{n}}{d t}(t)\right\rangle d t=\int_{0}^{T}\left\langle F_{n}\left(t, u_{n}(t), \mathcal{T}_{n} u_{n}(t)\right), \frac{d u_{n}}{d t}(t)\right\rangle d t
$$

Since $u_{n}^{0}=\eta_{n}(0) \in \operatorname{dom}\left(\Phi_{n}\right)$, we infer that $\frac{d u_{n}}{d t}$ belongs to $L^{2}(0, T, X)$ and $t \mapsto \Phi_{n}\left(u_{n}(t)\right)$ is absolutely continuous (see [6, Theorem 3.6]). Therefore for a.e. $t \in(0, T), \frac{d}{d t} \Phi\left(u_{n}(t)\right)=\left\langle D \Phi u_{n}(t), \frac{d u_{n}}{d t}(t)\right\rangle($ see 
4. Proposition 17.2.5]). From (3.2), 4.14) yields

$$
\begin{aligned}
\int_{0}^{T}\left\|\frac{d u_{n}}{d t}(t)\right\|^{2} d t & =-\Phi_{n}\left(u_{n}(T)\right)+\Phi_{n}\left(u_{n}^{0}\right)+\int_{0}^{T}\left\langle F_{n}\left(t, u_{n}(t), \mathcal{T}_{n} u_{n}(t)\right), \frac{d u_{n}}{d t}(t)\right\rangle d t \\
\leq & -\inf _{v \in L^{2}(\Omega), n \in \mathbb{N}} \Phi_{n}(v)+\sup _{n \in \mathbb{N}} \Phi_{n}\left(u_{n}^{0}\right) \\
& +\left(\int_{0}^{T}\left\|F_{n}\left(t, u_{n}(t), \mathcal{T}_{n} u_{n}(t)\right)\right\|_{X}^{2}\right)^{\frac{1}{2}}\left(\int_{0}^{T}\left\|\frac{d u_{n}}{d t}(t)\right\|_{X}^{2}\right)^{\frac{1}{2}} \cdot \\
\leq C\left(C_{\text {trace }}\right) \sup _{n \in \mathbb{N}}\left\|\phi_{n}\right\|_{L_{\mathcal{H}_{N-1}}^{2}(\partial \Omega)}^{2}+\sup _{n \in \mathbb{N}} \Phi_{n}\left(u_{n}^{0}\right) & +\left(\int_{0}^{T}\left\|F_{n}\left(t, u_{n}(t), \mathcal{T}_{n} u_{n}(t)\right)\right\|_{X}^{2}\right)^{\frac{1}{2}}\left(\int_{0}^{T}\left\|\frac{d u_{n}}{d t}(t)\right\|_{X}^{2}\right)^{\frac{1}{2}} .
\end{aligned}
$$

Hence, from 4.13$],\left(\mathrm{H}_{1}\right)$, and $\left(\mathrm{H}_{2}\right)$, we infer that there exists a positive constant $C$ such that

$$
\int_{0}^{T}\left\|\frac{d u_{n}}{d t}(t)\right\|_{X}^{2} d t \leq C\left(1+\left(\int_{0}^{T}\left\|\frac{d u_{n}}{d t}(t)\right\|_{X}^{2} d t\right)^{\frac{1}{2}}\right)
$$

from which we deduce 4.11.

Step 2. We prove that there exist $u \in C([0, T], X)$, and a subsequence of $\left(u_{n}\right)_{n \in \mathbb{N}}$ not relabeled, satisfying $u_{n} \rightarrow u$ in $C([0, T], X)$ equipped with its uniform norm.

We apply the Ascoli-Arzela compactness theorem. From $[4.9],\left(u_{n}\right)_{n \in \mathbb{N}}$ is bounded in $C([0, T], X)$. Moreover, for $s<t,(s, t) \in[0, T]$, we have

$$
\left\|u_{n}(t)-u_{n}(s)\right\|_{X} \leq \int_{s}^{t}\left\|\frac{d u_{n}}{d t}(\tau)\right\|_{X} d \tau \leq(t-s)^{\frac{1}{2}}\left\|\frac{d u_{n}}{d t}\right\|_{L^{2}(0, T, X)} \leq(t-s)^{\frac{1}{2}} \sup _{n \in \mathbb{N}}\left\|\frac{d u_{n}}{d t}\right\|_{L^{2}(0, T, X)}
$$

which, according to 4.11, provides the equicontinuity of the sequence $\left(u_{n}\right)_{n \in \mathbb{N}}$. It remains to establish for each $t \in[0, T]$, the relative compactness in $X$ of the set $E_{t}:=\left\{u_{n}(t): n \in \mathbb{N}\right\}$. For $t=0$ there is nothing to prove because of hypothesis $\left(\mathrm{H}_{3}\right)$ on the initial condition. We are going to prove the relative compactness of $E_{t}$ for $\left.\left.t \in\right] 0, T\right]$.

From Theorem 3.1. $u_{n}$ satisfies $\left[\mathrm{S}_{4}\right)$, then possesses a right derivative at each $\left.\left.t \in\right] 0, T\right]$ (at $t=T$, this is due to the fact that $u_{n}$ exists in $C([0,+\infty), X)$ so that the right derivative of $u_{n}$ at $t=T$ is nothing but the right derivative of the restriction of $u_{n}$ to $\left.[0, T]\right)$. Moreover

$$
\left.\left.\frac{d u_{n}^{+}}{d t}(t)+D \Phi_{n}\left(u_{n}(t)\right)=F_{n}\left(t, u_{n}(t), \mathcal{T}_{n} u_{n}(t)\right) \text { for all } t \in\right] 0, T\right]
$$

Taking $u_{n}(t)$ as a test function, we infer that for all $\left.\left.t \in\right] 0, T\right]$

$$
\left\langle\frac{d u_{n}^{+}}{d t}(t), u_{n}(t)\right\rangle+\left\langle D \Phi_{n}\left(u_{n}(t)\right), u_{n}(t)\right\rangle=\left\langle F_{n}\left(t, u_{n}(t), \mathcal{T}_{n} u_{n}(t)\right), u_{n}(t)\right\rangle
$$

hence, according to the Green formula and to the fact that $u_{n}(t) \in \operatorname{dom} D \Phi_{n}$ for all $\left.\left.t \in\right] 0, T\right]$, we deduce

$$
\begin{aligned}
& \int_{\Omega} D_{\xi} W_{n}\left(x, \nabla u_{n}(t)\right) \cdot \nabla u_{n}(t) d x \\
& =\int_{\partial \Omega} D_{\xi} W_{n}\left(x, \nabla u_{n}(t)\right) \cdot \mathbf{n} u_{n}(t) d \mathcal{H}_{N-1}-\int_{\Omega} \frac{d u_{n}^{+}}{d t}(t) u_{n}(t) d x+\int_{\Omega} F_{n}\left(t, u_{n}(t), \mathcal{T}_{n} u_{n}(t)\right) u_{n}(t) d x \\
& =\int_{\partial \Omega}\left(\phi_{n}-a_{0, n} u_{n}(t)\right) u_{n}(t) d \mathcal{H}_{N-1}-\int_{\Omega} \frac{d u_{n}^{+}}{d t}(t) u_{n}(t) d x+\int_{\Omega} F_{n}\left(t, u_{n}(t), \mathcal{T}_{n} u_{n}(t)\right) u_{n}(t) d x \\
& \leq \int_{\partial \Omega} \phi_{n} u_{n}(t) d \mathcal{H}_{N-1}-\int_{\Omega} \frac{d u_{n}^{+}}{d t}(t) u_{n}(t) d x+\int_{\Omega} F_{n}\left(t, u_{n}(t), \mathcal{T}_{n} u_{n}(t)\right) u_{n}(t) d x
\end{aligned}
$$

Take $\nu \in \mathbb{R}$ such that $0<\nu<\frac{2 \gamma}{C_{\text {trace }}}$ where $\gamma$ is the constant in $\left(\mathrm{D}_{3, n}\right)$, and $C_{\text {trace }}$ the constant of continuity of the trace operator. From the uniform strong convexity condition $\left(\mathrm{D}_{3, n}\right)$ and $(4.9)$, we 
deduce that for all $t \in] 0, T]$,

$$
\begin{aligned}
\gamma \int_{\Omega}\left|\nabla u_{n}(t)\right|^{2} d x \leq & \left\|\phi_{n}\right\|_{L_{\mathcal{H}_{N-1}}^{2}(\partial \Omega)}\left\|u_{n}(t)\right\|_{L_{\mathcal{H}_{N-1}}^{2}}(\partial \Omega) \\
& +\bar{\rho} \mathcal{L}_{N}(\Omega)^{\frac{1}{2}}\left(\left\|\frac{d u_{n}^{+}}{d t}(t)\right\|_{X}+\left\|F_{n}\left(t, u_{n}(t), \mathcal{T}_{n} u_{n}(t)\right)\right\|_{X}\right) \\
\leq & \frac{C_{\text {trace }}}{2 \nu}\left\|\phi_{n}\right\|_{L_{\mathcal{H}_{N-1}}^{2}}^{2}(\partial \Omega)+\frac{C_{\text {trace }} \nu}{2}\left\|u_{n}(t)\right\|_{H^{1}(\Omega)}^{2} \\
& +\bar{\rho} \mathcal{L}_{N}(\Omega)^{\frac{1}{2}}\left(\left\|\frac{d u_{n}^{+}}{d t}(t)\right\|_{X}+\left\|F_{n}\left(t, u_{n}(t), \mathcal{T}_{n} u_{n}(t)\right)\right\|_{X}\right) \\
\leq & \frac{C_{\text {trace }}}{2 \nu}\left\|\phi_{n}\right\|_{L_{\mathcal{H}_{N-1}}^{2}}^{2}(\partial \Omega)+\frac{C_{\text {trace }} \nu}{2}\left(\int_{\Omega}\left|\nabla u_{n}(t)\right|^{2} d x+\bar{\rho}^{2} \mathcal{L}_{N}(\Omega)\right) \\
& +\bar{\rho} \mathcal{L}_{N}(\Omega)^{\frac{1}{2}}\left(\left\|\frac{d u_{n}^{+}}{d t}(t)\right\|_{X}+\left\|F_{n}\left(t, u_{n}(t), \mathcal{T}_{n} u_{n}(t)\right)\right\|_{X}\right) .
\end{aligned}
$$

We infer that

$$
\begin{aligned}
\left(\gamma-\frac{C_{\text {trace }} \nu}{2}\right) \int_{\Omega}\left|\nabla u_{n}(t)\right|^{2} d x & \leq \frac{C_{\text {trace }}}{2 \nu} \sup _{n}\left\|\phi_{n}\right\|_{L_{\mathcal{H}^{N-1}}^{2}(\partial \Omega)}^{2}+\frac{C_{\text {trace }} \nu}{2} \bar{\rho}^{2} \mathcal{L}^{N}(\Omega) \\
& +\bar{\rho} \mathcal{L}^{N}(\Omega)^{\frac{1}{2}}\left(\left\|\frac{d u_{n}^{+}}{d t}(t)\right\|_{X}+\sup _{n \in \mathbb{N}}\left\|F_{n}\left(t, u_{n}(t), \mathcal{T}_{n} u_{n}(t)\right)\right\|_{X}\right) .
\end{aligned}
$$

From $4.12\left(\mathrm{H}_{7}\right)$, and $\left(\mathrm{H}_{1}\right)$, estimate 4.16$)$ and 4.9 yield that $\left(u_{n}(t)\right)_{n \in \mathbb{N}}$ is bounded in $H^{1}(\Omega)$ provided that we establish

$$
\sup _{n}\left\|\frac{d u_{n}^{+}}{d t}(t)\right\|_{X}<+\infty .
$$

Then, assuming (4.17), we conclude to the compactness of the set $E_{t}$ from the compact embedding $H^{1}(\Omega) \rightarrow L^{2}(\Omega)$. To prove (4.17) set $G_{n}(t):=F_{n}\left(t, u_{n}(t), \mathcal{T}_{n} u_{n}(t)\right)$. In order to apply Lemma C.1 of the Appendix, we start by establishing the following estimate on the total variation $\operatorname{Var}\left(G_{n},[0, T]\right)$ of $G_{n}$ in $[0, T]$ :

$$
\operatorname{Var}\left(G_{n},[0, T]\right)=\int_{0}^{T}\left\|\frac{G_{n}}{d t}(t)\right\|_{L^{2}(\Omega)} d t \leq C\left(1+\int_{0}^{T}\left\|\frac{d u_{n}}{d t}(t)\right\|_{L^{2}(\Omega)} d t\right)
$$

where $C$ is a nonnegative constant which does not depend on $n$. According to the structure of $F_{n}$, from 4.10 and $\left(\mathrm{H}_{6}\right)$ and noticing that from Lemma 2.1, $0 \leq \mathcal{T}_{n} u_{n} \leq \bar{y}_{n}(T) \leq \bar{\rho}$, we have

$$
\begin{aligned}
\left\|G_{n}(t)-G_{n}(s)\right\|_{X} \leq & \bar{g} \bar{h}\left\|r_{n}(t)-r_{n}(s)\right\|_{L^{2}\left(\Omega, \mathbb{R}^{l}\right)}+\left\|q_{n}(t)-q_{n}(s)\right\|_{L^{2}(\Omega)} \\
& +\sup _{n \in \mathbb{N}}\left\|r_{n}\right\|_{\infty}\left\|g_{n}\left(u_{n}(t)\right) \cdot h_{n}\left(\mathcal{T}_{n} u_{n}(t)\right)-g_{n}\left(u_{n}(s)\right) \cdot h_{n}\left(\mathcal{T}_{n} u_{n}(s)\right)\right\|_{L^{2}(\Omega)} \\
\leq & \bar{g} \bar{h} \int_{s}^{t}\left\|\frac{d r_{n}}{d t}(\tau, \cdot)\right\|_{L^{2}\left(\Omega, \mathbb{R}^{l}\right)} d \tau+\int_{s}^{t}\left\|\frac{d q_{n}}{d t}(\tau, \cdot)\right\|_{L^{2}(\Omega)} d \tau \\
& +\sup _{n \in \mathbb{N}}\left\|r_{n}\right\|_{\infty}\left\|g_{n}\left(u_{n}(t)\right) \cdot h_{n}\left(\mathcal{T}_{n} u_{n}(t)\right)-g_{n}\left(u_{n}(s)\right) \cdot h_{n}\left(\mathcal{T}_{n} u_{n}(s)\right)\right\|_{L^{2}(\Omega)} .
\end{aligned}
$$

On the other hand, from (4.2), 4.5) and (4.10), we infer that

$$
\begin{aligned}
& \left\|g_{n}\left(u_{n}(t)\right) \cdot h_{n}\left(\mathcal{T}_{n} u_{n}(t)\right)-g_{n}\left(u_{n}(s)\right) \cdot h_{n}\left(\mathcal{T}_{n} u_{n}(s)\right)\right\|_{L^{2}(\Omega)} \\
& \leq \bar{g} L_{[0, \bar{\rho}]}^{\prime}\left\|\mathcal{T}_{n} u_{n}(t)-\mathcal{T} u_{n}(s)\right\|_{L^{2}(\Omega)}+\bar{h} L_{[0, \bar{\rho}]}\left\|u_{n}(t)-u_{n}(s)\right\|_{L^{2}(\Omega)} \\
& \leq g L_{[0, \bar{\rho}]}^{\prime} \int_{s}^{t}\left(\left\|\frac{d u_{n}}{d \sigma}(\cdot)\right\|_{L^{2}(\Omega)} \star \mu_{n}\right)(\sigma) d \sigma+\bar{h} L_{[0, \bar{\rho}]} \int_{s}^{t}\left\|\frac{d u_{n}}{d \sigma}(\sigma)\right\|_{L^{2}(\Omega)} d \sigma .
\end{aligned}
$$

Combining (4.19) with 4.20), from (4.3) we deduce that

$$
\int_{0}^{T}\left\|\frac{G_{n}}{d \sigma}(\sigma)\right\|_{L^{2}(\Omega)} d \sigma \leq C\left(1+\int_{0}^{T}\left(\left\|\frac{d u_{n}}{d \sigma}(\cdot)\right\|_{L^{2}(\Omega)} \star \mu_{n}\right)(\sigma) d \sigma+\int_{0}^{T}\left\|\frac{d u_{n}}{d \sigma}(\sigma)\right\|_{L^{2}(\Omega)} d \sigma\right) .
$$


Since $u_{n} \in C_{\eta_{n}}\left((-\infty, T], L^{2}(\Omega)\right)$, according to Lemma 2.2 , we have

$$
\int_{0}^{T}\left(\left\|\frac{d u_{n}}{d \sigma}(\cdot)\right\|_{L^{2}(\Omega)} \star \mu_{n}\right)(\sigma) d \sigma \leq \int_{-\infty}^{0}\left\|\frac{d \eta_{n}}{d \sigma}(\sigma)\right\|_{L^{2}(\Omega)} d \sigma+\int_{0}^{T}\left\|\frac{d u_{n}}{d \sigma}(\sigma)\right\|_{L^{2}(\Omega)} d \sigma
$$

so that, from (4.4),

$$
\int_{0}^{T}\left(\left\|\frac{d u_{n}}{d \sigma}(\cdot)\right\|_{L^{2}(\Omega)} \star \mu_{n}\right)(\sigma) d \sigma \leq C\left(1+\int_{0}^{T}\left\|\frac{d u_{n}}{d \sigma}(\sigma)\right\|_{L^{2}(\Omega)} d \sigma\right) .
$$

Estimate 4.18 is obtained by Collecting 4.21 and 4.22 .

Applying Lemma C.1. from (4.18), we deduce that

$$
\left\|\frac{d u_{n}^{+}}{d t}(t)\right\|_{X} \leq\left(C+\left(C+\frac{1}{t}\right) \int_{0}^{T}\left\|\frac{d u_{n}}{d t}(t)\right\|_{X} d t\right)
$$

and 4.17) follows from 4.11.

Step 3. We assert that $\frac{d u_{n}}{d t} \rightarrow \frac{d u}{d t}$ weakly in $L^{2}(0, T, X)$ for a non relabeled subsequence, and that $0 \leq u \leq \bar{\rho}$. The first claim is a straightforward consequence of 4.11) and Step 2. The second one follows easily from inequality $0 \leq u_{n} \leq \bar{\rho}$ and $u_{n} \rightarrow u$ in $C([0, T], X)$.

Step 4. We prove that $u$, extended by $\eta$ in $(-\infty, 0]$, is the unique solution of $(\mathcal{P})$. The proof of

$$
\frac{d u}{d t}(t)+\partial \Phi(u(t)) \ni F(t, u(t), \mathcal{T} u(t)) \text { for a.e. } t \in(0, T)
$$

mimics that of [2, Theorem 3.1] so that we give a sketch of the proof. The only trickier result is the following version of [2, Lemma4.2] whose proof is postponed to the last step.

Lemma 4.1. The functional $G_{n}=F_{n}\left(\cdot, u_{n}, \mathcal{T}_{n} u_{n}\right)$ weakly converges in $L^{2}\left(0, T, L^{2}(\Omega)\right)$ to the functional $G$ defined by $G(t)=F(t, u(t), \mathcal{T} u(t))$ where $F(t, u(t), \mathcal{T} u(t))=r(t) \odot h(\mathcal{T} u(t)) \cdot g(u(t))+q(t)$.

From Step 2, there exists $u \in C([0, T], X)$ and a (non relabeled) subsequence such that $u_{n} \rightarrow u$ in $C([0, T], X)$. According to the Fenchel extremality condition (see [4, Proposition 9.5.1]) $\left(\mathcal{P}_{n}\right.$ ) is equivalent to

$$
\Phi_{n}\left(u_{n}(t)\right)+\Phi_{n}^{*}\left(G_{n}(t)-\frac{d u_{n}}{d t}(t)\right)+\left\langle\frac{d u_{n}}{d t}(t)-G_{n}(t), u_{n}(t)\right\rangle=0
$$

for a.e. $t \in(0, T)$ which, from the Legendre-Fenchel inequality, is equivalent to

$$
\int_{0}^{T}\left[\Phi_{n}\left(u_{n}(t)\right)+\Phi_{n}^{*}\left(G_{n}(t)-\frac{d u_{n}}{d t}(t)\right)\right] d t+\frac{1}{2}\left(\left\|u_{n}(T)\right\|^{2}-\left\|u_{n}^{0}\right\|^{2}\right)-\int_{0}^{T}\left\langle G_{n}(t), u_{n}(t)\right\rangle d t=0 .
$$

From $\left(\mathrm{H}_{3}\right)$ we have

$$
\left\|u_{n}^{0}\right\|_{X} \rightarrow\left\|u^{0}\right\|_{X}
$$

Combining $u_{n}(T)=u_{n}^{0}+\int_{0}^{T} \frac{d u_{n}}{d t}(t) d t$ with $\frac{d u_{n}}{d t} \rightarrow \frac{d u}{d t}$ in $L^{2}(0, T, X)$, we infer that

$$
\liminf _{n \rightarrow+\infty}\left\|u_{n}(T)\right\|^{2} \geq\|u(T)\|^{2} .
$$

From Lemma 4.1

$$
G_{n} \rightarrow G \text { weakly in } L^{2}(0, T, X) .
$$

From $\left(\mathrm{H}_{1}\right)$ the integral functionals $I_{\Phi_{n}}$ and $I_{\Phi_{n}^{*}}$ respectively defined in $L^{2}(0, \widetilde{T}, X)$ by $I_{\Phi_{n}}(v)=$ $\int_{0}^{T} \Phi_{n}(v(t)) d t$ and $I_{\Phi_{n}^{*}}(v)=\int_{0}^{T} \Phi_{n}^{*}(v(t)) d t$ Mosco-converge to the integrals functionals $I_{\Phi}$ and $I_{\Phi^{*}}$ respectively defined by $I_{\Phi}(v)=\int_{0}^{T} \Phi(v(t)) d t$ and $I_{\Phi^{*}}(v)=\int_{0}^{T} \Phi^{*}(v(t)) d t$ (for a proof consult [2, Lemma 4.1]). Hence, passing to the limit in (4.23), from (4.24), 4.25), 4.26), and Step 3 we obtain

$$
\int_{0}^{T}\left[\Phi(u(t))+\Phi^{*}\left(G(t)-\frac{d u}{d t}(t)\right)\right] d t+\frac{1}{2}\left(\|u(T)\|^{2}-\left\|u^{0}\right\|^{2}\right)-\int_{0}^{T}\langle G(t), u(t)\rangle d t \leq 0
$$


or equivalently

$$
\int_{0}^{T}\left[\Phi(u(t))+\Phi^{*}\left(G(t)-\frac{d u}{d t}(t)\right)+\left\langle\frac{d u}{d t}(t)-G(t), u(t)\right\rangle\right] d t \leq 0 .
$$

But from the Legendre-Fenchel inequality we have $\Phi(u(t))+\Phi^{*}\left(G(t)-\frac{d u}{d t}(t)\right)+\left\langle\frac{d u}{d t}(t)-G(t), u(t)\right\rangle \geq$ 0 , so that 4.27 yields that for a.e. $t \in(0, T), \Phi(u(t))+\Phi^{*}\left(G(t)-\frac{d u}{d t}(t)\right)+\left\langle\frac{d u}{d t}(t)-G(t), u(t)\right\rangle=0$ which is, according to [4, Proposition 9.5.1], equivalent to

$$
\frac{d u}{d t}(t)+\partial \Phi(u(t)) \ni G(t) \text { for a.e. } t \in(0, T) .
$$

We have already proved that $0 \leq \eta \leq \bar{\rho}$ in Step 1. It remains to establish that $\eta(0) \in \operatorname{dom}(\Phi)$. From $\left(\mathrm{H}_{1}\right),\left(\mathrm{H}_{2}\right)$, and $\left(\mathrm{H}_{3}\right)$, we infer that

$$
\Phi(\eta(0)) \leq \liminf _{n \rightarrow+\infty} \Phi_{n}\left(\eta_{n}(0)\right) \leq \sup _{n \in \mathbb{N}} \Phi_{n}\left(\eta_{n}(0)\right)<+\infty,
$$

which prove the thesis.

\section{Step 5.}

Proof of Lemma 4.1. The fact that $\mathcal{T}$ is well defined follows straightforwardly from the pointwise limit $\mathcal{T}_{n} u(t) \rightarrow \mathcal{T} u(t)$ in $L^{2}(\Omega)$ ensured by $\left(\mathrm{H}_{4}\right)$; indeed write $\mathcal{T}_{n} u(t)=\int_{\mathbb{R}} \mathbb{1}_{(-\infty, t]} \widetilde{u}_{t} d \mathbf{m}_{t}^{n}$ and $\mathcal{T} u(t)=$ $\int_{\mathbb{R}} \mathbb{1}_{(-\infty, t]} \widetilde{u}_{t} d \mathbf{m}_{t}$ where $\widetilde{u}_{t}$ defined by 2.1 belongs to $C_{c}\left(\mathbb{R}, L^{2}(\Omega)\right)$.

Recall that $G_{n}(t)=H_{n}(t)+q_{n}(t)$ where

$$
H_{n}(t)(x)=r_{n}(t, x) \odot h_{n}\left(\mathcal{T}_{n} u_{n}(t, x)\right) \cdot g_{n}\left(u_{n}(t, x)\right)=r_{n}(t, x) \cdot h_{n}\left(\mathcal{T}_{n} u_{n}(t, x)\right) \odot g_{n}\left(u_{n}(t, x)\right),
$$

Since $q_{n} \rightarrow q$ in $L^{2}(0, T, X)$, we are reduced to prove that $H_{n} \rightarrow H$ in $L^{2}(0, T, X)$ where

$$
H(t)(x)=r(t, x) \odot h(\mathcal{T} u(t, x)) \cdot g(u(t, x))=r(t, x) \cdot h(\mathcal{T} u(t, x)) \odot g(u(t, x)) .
$$

Hence, since $r_{n} \rightarrow r$ in $L^{2}(0, T, X)$, it suffices to establish that

$$
h_{n}\left(\mathcal{T}_{n} u_{n}\right) \odot g_{n}\left(u_{n}\right) \rightarrow h\left(\mathcal{T}_{n} u\right) \odot g(u) \text { strongly in } L^{2}\left(0, T, X^{l}\right)
$$

where $X^{l}$ denotes the space $L^{2}\left(\Omega, \mathbb{R}^{l}\right)$. We have ${ }^{6}$

$$
\begin{aligned}
& \quad\left\|h_{n}\left(\mathcal{T}_{n} u_{n}(t)\right) \odot g_{n}\left(u_{n}(t)\right)-h(\mathcal{T} u(t)) \odot g(u(t))\right\|_{X^{l}} \\
& \leq\left\|h_{n}\left(\mathcal{T}_{n} u_{n}(t)\right) \odot g_{n}\left(u_{n}(t)\right)-h_{n}\left(\mathcal{T}_{n} u_{n}(t)\right) \odot g(u(t))\right\|_{X^{l}} \\
& \quad+\left\|h_{n}\left(\mathcal{T}_{n} u_{n}(t)\right) \odot g(u(t))-h(\mathcal{T} u(t)) \odot g(u(t))\right\|_{X^{l}} \\
& \leq \bar{h}\left\|g_{n}\left(u_{n}(t)\right)-g(u(t))\right\|_{X^{l}}+\bar{g}\left\|h_{n}\left(\mathcal{T}_{n} u_{n}(t)\right)-h(\mathcal{T} u(t))\right\|_{X^{l}} \\
& \leq \bar{h} L_{[0, \bar{\rho}]}\left\|u_{n}(t)-u(t)\right\|_{X}+\bar{h}\left\|g_{n}(u(t))-g(u(t))\right\|_{X^{l}} \\
& \quad+\bar{g} L_{[0, \bar{\rho}]}^{\prime}\left\|\mathcal{T}_{n} u_{n}(t)-\mathcal{T} u(t)\right\|_{X}+\bar{g}\left\|h_{n}(\mathcal{T} u(t))-h(\mathcal{T} u(t))\right\|_{X^{l}} .
\end{aligned}
$$

Hence, to prove the claim we need to establish that

$$
\begin{gathered}
\int_{0}^{T}\left\|g_{n}(u(t))-g(u(t))\right\|_{X^{l}}^{2} d t \rightarrow 0, \int_{0}^{T}\left\|h_{n}(\mathcal{T} u(t))-h(\mathcal{T} u(t))\right\|_{X^{l}}^{2} d t \rightarrow 0 \\
\int_{0}^{T}\left\|u_{n}(t)-u(t)\right\|_{X}^{2} d t \rightarrow 0 \\
\int_{0}^{T}\left\|\mathcal{T}_{n} u_{n}(t)-\mathcal{T} u(t)\right\|_{X}^{2} d t \rightarrow 0 .
\end{gathered}
$$

The two convergences in 4.28 follow easily from $\left(\mathrm{H}_{5}\right)$ and the Lebesgue dominated convergence theorem. Convergence (4.29) is straitghforward. We establish (4.30). From 2.3), we have

$$
\begin{aligned}
\left\|\mathcal{T}_{n} u_{n}-\mathcal{T} u\right\|_{L^{2}(0, T, X)} & \leq\left\|\mathcal{T}_{n}\left(u_{n}-u\right)\right\|_{L^{2}(0, T, X)}+\left\|\mathcal{T}_{n} u-\mathcal{T} u\right\|_{L^{2}(0, T, X)} \\
& \leq 2 T^{\frac{1}{2}}\left\|u_{n}-u\right\|_{C([0, T], X)}+\left\|\mathcal{T}_{n} u-\mathcal{T} u\right\|_{L^{2}(0, T, X)}
\end{aligned}
$$

\footnotetext{
${ }^{6}$ To simplify the notation we write $g(v(t))$ for the function $x \mapsto g(v(t, x))$, idem for $h(v(t)), g_{n}(v(t))$ and $h_{n}(v(t))$.
} 
Hence it remains to prove that $\left\|\mathcal{T}_{n} u-\mathcal{T} u\right\|_{L^{2}(0, T, X)} \rightarrow 0$. The claim follows from the pointwise convergence $\mathcal{T}_{n} u(t) \rightarrow \mathcal{T} u(t)$ in $L^{2}(\Omega)$ for a.e. $t \in(0, T)$, $(2.2)$, and the Lebesgue dominated convergence theorem. This ends the proof of Lemma 4.1 .

This completes the proof of Theoram 4.1 .

Remark 4.1. It is easy to check that $\left(\mathrm{H}_{4}\right)$ holds for the measures described in the three examples $2.1,2.2$ and 2.3 in the following situations

1) $\mathbf{m}_{t}^{n}=\sum_{k \in \mathbb{N}} \mathbf{d}_{k} \delta_{t-\tau_{k, n}}$ with $\tau_{k, n} \rightarrow \tau_{k}$ in $\mathbb{R}_{+} ;$then $\mathbf{m}_{t}=\sum_{k \in \mathbb{N}} \mathbf{d}_{k} \delta_{t-\tau_{k}}$;

2) $\mathbf{m}_{t}^{n}=\frac{1}{\# \tau} \delta_{t-\tau_{n}(\cdot)}$ where $\#\left(\tau_{n}(\mathbb{R})\right)$ is assumed to be a constant, denoted by $\# \tau$, and $\tau_{n} \rightarrow \tau$ pointwise in $\Omega$; then $\mathbf{m}_{t}=\frac{1}{\# \tau} \delta_{t-\tau(\cdot)}$;

3) $\mathbf{m}_{t}^{n}=\mathcal{K}_{n}(\cdot, t-\sigma) d \sigma$ where $\mathcal{K}_{n}(\cdot, \sigma) \rightarrow \mathcal{K}(\cdot, \sigma)$ in $L^{2}(\Omega)$, with the domination property: there exists $\mathcal{J} \in L^{1}(0,+\infty)$ such that $\left\|\mathcal{K}_{n}(\cdot, \sigma)\right\|_{L^{2}(\Omega)} \leq \mathcal{J}(\sigma)$ a.e.. Then $\mathbf{m}_{t}=\mathcal{K}(\cdot, t-\sigma) d \sigma$.

4.2. Non stability at the limit for the reaction functional: convergence with mixing effect between growth rates and time delays at the limit. We substitute for $\left(\mathrm{H}_{4}\right)$ a global weak convergence $\left(\mathrm{H}_{4}^{\prime}\right)$ of the product of the growth rate $r_{n}$ with the time delays term $h_{n}\left(\mathcal{T}_{n} \cdot\right)$. The structure of the reaction functional is then no longer preserved at the limit. This phenomena occurs in stochastic homogenization when the time-delays are strongly oscillating through the space variable. It will be illustrated in Section 5 .

Theorem 4.2 (Mixing effect). Assume that $\left(W_{n}\right)_{n \in \mathbb{N}}$ satisfies conditions $\left(\mathrm{D}_{1, n}\right),\left(\mathrm{D}_{2, n}\right),\left(\mathrm{D}_{3, n}\right)$, and that the sequence of DCP-structured reaction functionals $\left(F_{n}\right)_{n \in \mathbb{N}}$ satisfies $(\mathrm{SC} 1)$, $(\mathrm{SC} 2)$, (SC3) and (SC4), Let $u_{n}$ be the unique solution of the delays problem

$$
\left(\mathcal{P}_{n}\right)\left\{\begin{array}{l}
\frac{d u_{n}}{d t}(t)+D \Phi_{n}\left(u_{n}(t)\right)=F_{n}\left(t, u_{n}(t), \mathcal{T}_{n} u_{n}(t)\right) \text { for a.e. } t \in(0, T), \\
u_{n}(t)=\eta_{n}(t) \text { for all } t \in(-\infty, 0] .
\end{array}\right.
$$

Assume hypotheses $\left(\mathrm{H}_{1}\right),\left(\mathrm{H}_{2}\right),\left(\mathrm{H}_{3}\right),\left(\mathrm{H}_{7}\right)$ and

$\left(\mathrm{H}_{4}^{\prime}\right)$ there exists a function $r_{\mathcal{T}}: C_{c}\left((-\infty, T], L^{2}(\Omega)\right) \rightarrow L^{2}\left(0, T, L^{2}(\Omega)\right)$ such that $r_{n} \odot h_{n}\left(\mathcal{T}_{n} \psi\right) \rightarrow$ $r_{\mathcal{T}}(\psi){ }^{7}$ weakly in $L^{2}\left(0, T, L^{2}\left(\Omega ; \mathbb{R}^{l}\right)\right)$ for all $\psi \in C_{c}\left((-\infty, T], L^{2}(\Omega)\right)$ satisfying $0 \leq \psi \leq \bar{\rho}$ and support $\left(\psi_{\lfloor(-\infty, 0]}\right) \subset \operatorname{support}(\eta)$;

$\left(\mathrm{H}_{5}^{\prime}\right) g_{n}$ pointwise converges to $g$ and $\sup _{n \in \mathbb{N}}\left|h_{n}(0)\right|<+\infty$;

$\left(\mathrm{H}_{6}^{\prime}\right) \bar{r}=\sup _{n \in \mathbb{N}}\left\|r_{n}\right\|_{L^{\infty}\left([0,+\infty) \times \mathbb{R}^{N}, \mathbb{R}^{l}\right)}<+\infty ;$

Then $u_{n}$ uniformly converges in $C\left([0, T], L^{2}(\Omega)\right)$ to some function $u$ whose extension by $\eta$ in $(-\infty, 0]$, is the unique solution of the problem

$$
(\mathcal{P})\left\{\begin{array}{l}
\frac{d u}{d t}(t)+\partial \Phi(u(t)) \ni r_{\mathcal{T}}(u)(t) \cdot g(u(t))+q(t) \text { for a.e. } t \in(0, T) \\
u(t)=\eta(t), 0 \leq \eta(t) \leq \bar{\rho}:=\sup _{n \in \mathbb{N}} \bar{y}_{n}(T) \text { for all } t \in(-\infty, 0], \eta(0) \in \operatorname{dom}(\Phi) .
\end{array}\right.
$$

Moreover, $\frac{d u_{n}}{d t} \rightarrow \frac{d u}{d t}$ weakly in $L^{2}\left(0, T, L^{2}(\Omega)\right)$, and $0 \leq u \leq \bar{\rho}:=\sup _{n \in \mathbb{N}} \bar{y}_{n}(T)$.

Proof. All the proof of Theorem 4.1 remains valid, excepted Step 4, Lemma 4.1. We only have to substitute Lemma 4.2 below for Lemma 4.1

Lemma 4.2. The functional $G_{n}=F_{n}\left(\cdot, u_{n}, \mathcal{T}_{n} u_{n}\right)$ weakly converges in $L^{2}(0, T, X)$ to the functional $G$ defined for all $t \in[0, T]$ by $G(t)=r_{\mathcal{T}}(u)(t) \cdot g(u(t))+q(t)$.

\footnotetext{
${ }^{7}$ We write $r_{n} \odot h_{n}\left(\mathcal{T}_{n} \psi\right)$ to denote the function defined in $L^{2}\left(0, T, L^{2}(\Omega)\right)$ by $(t, x) \mapsto r_{n}(t, x) \odot h_{n}\left(\mathcal{T}_{n} \psi(t, x)\right)$.
} 
Proof of Lemma 4.2. Recall that $G_{n}(t)=H_{n}(t)+q_{n}(t)$ where $H_{n}(t)(x)=r_{n}(t, x) \odot h_{n}\left(\mathcal{T}_{n} u_{n}(t, x)\right)$. $g_{n}\left(u_{n}(t, x)\right)$. Since $q_{n} \rightarrow q$ in $L^{2}(0, T, X)$, it remains to prove that $H_{n} \rightarrow H$ in $L^{2}(0, T, X)$ where $H(t)=r_{\mathcal{T}}(u)(t) \cdot g(u(t))$. From $\left(\mathrm{H}_{5}^{\prime}\right)$, 4.2, 4.6), and according to Step 2 in the proof of Theorem 4.1. it is easy to show that $g_{n}\left(u_{n}\right) \rightarrow g(u)$ strongly in $L^{2}\left(0, T, L^{2}\left(\Omega ; \mathbb{R}^{l}\right)\right)$. To conclude, we prove that $r_{n} \odot h_{n}\left(\mathcal{T}_{n} u_{n}\right) \rightarrow r_{\mathcal{T}}(u)$ weakly in $L^{2}\left(0, T, L^{2}\left(\Omega ; \mathbb{R}^{l}\right)\right)$. We have

$$
r_{n} \odot h_{n}\left(\mathcal{T}_{n} u_{n}\right)=r_{n} \odot h_{n}\left(\mathcal{T}_{n} u\right)+r_{n}(t) \odot\left(h_{n}\left(\mathcal{T}_{n} u_{n}\right)-h_{n}\left(\mathcal{T}_{n} u\right)\right)
$$

From $(4.2), 2.3],\left(\mathrm{H}_{6}^{\prime}\right)$ and the fact that $0 \leq \mathcal{T}_{n} u_{n} \leq \bar{\rho}, 0 \leq \mathcal{T}_{n} u \leq \bar{\rho}$, we have

$$
\left\|r_{n}(t) \odot\left(h_{n}\left(\mathcal{T}_{n} u_{n}\right)-h_{n}\left(\mathcal{T}_{n} u\right)\right)\right\|_{L^{2}\left(0, T, X^{l}\right)} \leq \bar{r} T^{\frac{1}{2}} \mathcal{L}_{N}(\Omega)^{\frac{1}{2}} L_{[0, \bar{\rho}]}^{\prime}\left\|u_{n}-u\right\|_{C((-\infty, T], X)},
$$

hence $r_{n}(t) \odot\left(h_{n}\left(\mathcal{T}_{n} u_{n}\right)-h_{n}\left(\mathcal{T}_{n} u\right)\right)$ strongly converges to 0 in $L^{2}\left(0, T, L^{2}\left(\Omega ; \mathbb{R}^{l}\right)\right)$. The conclusion then follows from 4.31 and hypothesis $\left(\mathrm{H}_{4}^{\prime}\right)$ by taking $\psi=u$ as a test function.

Remark 4.2. In practice, the difficulty is to establish the convergence in hypothesis $\left(\mathrm{H}_{4}^{\prime}\right)$ We will obtain $\left(\mathrm{H}_{4}^{\prime}\right)$ in the context of stochastic homogenization with certain functions $h$, then illustrating the interplay at the limit, between the growth rate $r_{n}$, and the time delays modeled by $h\left(\mathcal{T}_{n} \psi\right)$.

Remark 4.3. Theorems 4.1 and 4.2 can be generalized without difficulty to differential inclusions $\left(\mathcal{P}_{n}\right)$ under minor additional conditions (see [2, Remark 4.1]). Due to the fact that the graph limit of a sequence of single valued maximal monotone operator may be multivalued, the limit problem in Theorems 4.1 and 4.2 is a differential inclusion. In some cases the diffusion term of the limit problem is a single valued subdifferential as seen in Section 5 in the framework of homogenization (see condition (GD) on the Fenchel conjugate of $W(x, \cdot))$.

\section{Stochastic homogenization of Distributed Delays REACTION DifFusion PRoblems}

We place this section within the framework of stochastic homogenization introduced in 2] (see also 4. Section 17.4.5]). Recall that the triple $\left(\Sigma, \mathcal{A}, \mathbf{P},\left(T_{z}\right)_{z \in \mathbb{Z}^{N}}\right)$ is a discrete dynamical system made up of a probabilistic space $(\Sigma, \mathcal{A}, \mathbf{P})$ equipped with a group $\left(T_{z}\right)_{z \in \mathbb{Z}^{N}}$ of $\mathbf{P}$-preserving transformations. We denote by $\mathcal{I}$ the $\sigma$-algebra of invariant sets of $\mathcal{A}$ by $\left(T_{z}\right)_{z \in \mathbb{Z}^{N}}$ and, for every $\mathbf{h}$ in the space $L_{\mathbf{P}}^{1}(\Sigma)$ of $\mathbf{P}$-integrable functions, by $\mathbf{E}^{\mathcal{I}} \mathbf{h}$ the conditional expectation of $\mathbf{h}$ with respect to $\mathcal{I}$. Theorem 5.1, states that a mixing phenomena appears for the homogenized reaction functional between the various growths rate and time delays entering the structure of the random reaction functional. This result is illustrated in Appendix E through the homogenization of a vector disease model and a delay logistic equation with immigration. In the first example, the growth rate of the uninfected population and the time delays coefficients of the homogenized problem are mixed in such a way that the effective distributed delays are associated with a measure incorporating the growth rate. In the second example, the homogenized problem may be interpreted as a diffuse delay logistic equation modeling the evolution of a density population spreading in an homogeneous environment, whose carrying capacity is function of the growth rate and time dependent.

5.1. The random diffusion part. The diffusion part, is that of Section 3 with random coefficients (see [2]). Just recall that the diffusion operator is the subdifferential of the random energy functional

$$
\Phi_{\varepsilon}(\omega, u)=\left\{\begin{array}{cl}
\widetilde{\Phi}_{\varepsilon}(\omega, u)+\frac{1}{2} \int_{\partial \Omega} a_{0} u d \mathcal{H}_{N-1}-\int_{\partial \Omega} \phi u d \mathcal{H}_{N-1} & \text { if } u \in H^{1}(\Omega) \\
+\infty & \text { otherwise, }
\end{array}\right.
$$

where

$$
\widetilde{\Phi}_{\varepsilon}(\omega, u)=\left\{\begin{array}{cl}
\int_{\Omega} W\left(\omega, \frac{x}{\varepsilon}, \nabla u\right) d x & \text { if } u \in H^{1}(\Omega) \\
+\infty & \text { otherwise. }
\end{array}\right.
$$

and $W$ is a random convex integrand $W: \Sigma \times \mathbb{R}^{N} \times \mathbb{R}^{N} \rightarrow \mathbb{R}$, covariant with respect to the dynamical system $\left(\Sigma, \mathcal{A}, \mathbf{P},\left(T_{z}\right)_{z \in \mathbb{Z}^{N}}\right)$. More precisely $W$ is a $\left(\mathcal{A} \otimes \mathcal{B}\left(\mathbb{R}^{N}\right) \otimes \mathcal{B}\left(\mathbb{R}^{N}\right), \mathcal{B}(\mathbb{R})\right)$-measurable function such that for every $\omega \in \Sigma, W(\omega, \cdot, \cdot)$, belongs to the class of functions mapping $\mathbb{R}^{N} \times \mathbb{R}^{N}$ to $\mathbb{R}$ satisfying 
conditions $\left(\mathrm{D}_{1, n}\right),\left(\mathrm{D}_{2, n}\right)$, and $\left(\mathrm{D}_{3, n}\right)$ for fixed $\omega$. The covariance property is stated as follows: for all $z \in \mathbb{Z}^{N}$

$$
W\left(T_{z} \omega, x, \xi\right)=W(\omega, x+z, \xi) \text { for a.e. } x \in \mathbb{R}^{N} \text {, for all } \xi \in \mathbb{R}^{N} \text {, and for } \mathbf{P} \text {-a.e. } \omega \in \Sigma \text {. }
$$

We also recall that for $\mathbf{P}$ a.e. $\omega \in \Sigma$, the functional $\Phi_{\varepsilon}(\omega, \cdot)$ Mosco-converges to the functional

$$
\Phi^{\text {hom }}(\omega, u)=\left\{\begin{array}{cl}
\widetilde{\Phi}^{\text {hom }}(\omega, u)+\frac{1}{2} \int_{\partial \Omega} a_{0} u d \mathcal{H}_{N-1}-\int_{\partial \Omega} \phi u d \mathcal{H}_{N-1} & \text { if } u \in H^{1}(\Omega) \\
+\infty & \text { otherwise. }
\end{array}\right.
$$

where

$$
\widetilde{\Phi}^{h o m}(\omega, u)=\left\{\begin{array}{cl}
\int_{\Omega} W^{h o m}(\omega, \nabla u) d x & \text { if } u \in H^{1}(\Omega) \\
+\infty & \text { otherwise }
\end{array}\right.
$$

and $W^{\text {hom }}$ is given by

$$
\begin{aligned}
W^{h o m}(\omega, \xi) & =\lim _{n \rightarrow+\infty} \mathbf{E}^{\mathcal{I}} \inf \left\{\frac{1}{n^{N}} \int_{n Y} W(\omega, y, \xi+\nabla u(y)) d y: u \in H_{0}^{1}(n Y)\right\} \\
& =\inf _{n \in \mathbb{N}^{*}} \mathbf{E}^{\mathcal{I}} \inf \left\{\frac{1}{n^{N}} \int_{n Y} W(\omega, y, \xi+\nabla u(y)) d y: u \in H_{0}^{1}(n Y)\right\}
\end{aligned}
$$

If $\left(\Sigma, \mathcal{A}, \mathbf{P},\left(T_{z}\right)_{z \in \mathbb{Z}^{N}}\right)$ is ergodic, then $W^{\text {hom }}$ is deterministic and given for $\mathbf{P}$-a.s. $\omega \in \Sigma$ by

$$
\begin{aligned}
W^{h o m}(\xi) & =\lim _{n \rightarrow+\infty} \operatorname{Einf}\left\{\frac{1}{n^{N}} \int_{n Y} W(\omega, y, \xi+\nabla u(y)) d y: u \in H_{0}^{1}(n Y)\right\} \\
& =\inf _{n \in \mathbb{N}^{*}} \mathbf{E} \inf \left\{\frac{1}{n^{N}} \int_{n Y} W(\omega, y, \xi+\nabla u(y)) d y: u \in H_{0}^{1}(n Y)\right\} .
\end{aligned}
$$

We can not guarantee a priori that $W^{\text {hom }}(\omega, \cdot)$ is Gâteaux-differentiable. Hence $\partial_{\xi} W^{h o m}(\omega, \cdot)$ is possibly multivalued. Nevertheless, to shorten the notation, we write indifferently $\partial_{\xi} W^{\text {hom }}(\omega, \cdot)$ to denote the subdiffrential $\partial_{\xi} W^{h o m}(\omega, \cdot)$ or any of its elements. Under the following additional condition on the Fenchel conjugate of $\xi \mapsto W(\omega, x, \xi)$, the density $W^{\text {hom }}(\omega, \cdot)$ is Gâteaux differentiable for $\mathbf{P}$ a.e. $\omega \in \Sigma$, so that $\partial_{\xi} W^{\text {hom }}(\omega, \cdot)$ is univalent and reduced to a pointwise limit:

(GD) there exists $\gamma^{*}>0$ such that $\left\langle\xi_{1}^{*}-\xi_{2}^{*}, \xi^{1}-\xi^{2}\right\rangle \geq \gamma^{*}\left|\xi_{1}-\xi_{2}\right|^{2}$ for $\mathbf{P}$ a.e. $\omega \in \Sigma$, for a.e. $x \in \mathbb{R}^{N}$, for all $\left(\xi_{1}, \xi_{2}\right) \in \mathbb{R}^{N} \times \mathbb{R}^{N}$ and all $\left(\xi_{1}^{*}, \xi_{2}^{*}\right) \in \partial_{\xi} W^{*}\left(\omega, x, \xi_{1}\right) \times \partial_{\xi} W^{*}\left(\omega, x, \xi_{2}\right)$.

For a proof, we refer the reader to [4, Proposition 17.4.6].

5.2. The random reaction part. Consider a random DCP-structured reaction functional, namely, a functional

$$
F: \Sigma \times[0,+\infty) \times L^{2}(\Omega) \times L^{2}(\Omega) \rightarrow \mathbb{R}^{\Omega}
$$

defined by $F(\omega, t, u, v)(x)=f(\omega, t, x, u(x), v(x))$ where

$$
f: \Sigma \times[0,+\infty) \times \mathbb{R}^{N} \times \mathbb{R} \times \mathbb{R} \rightarrow \mathbb{R}
$$

is $\mathcal{A} \otimes \mathcal{B}(\mathbb{R}) \otimes \mathcal{B}\left(\mathbb{R}^{N}\right) \otimes \mathcal{B}(\mathbb{R})-\mathcal{B}(\mathbb{R})$ measurable and such that for $\mathbf{P}$-a.s. $\omega \in \Sigma, f(\omega, \cdot, \cdot, \cdot, \cdot)$ is a DCP-structured reaction function associated with $(r(\omega, \cdot), g, h, q(\omega, \cdot))$.

We assume that $r \in W^{1,2}\left(0, T, L_{\text {loc }}^{2}\left(\mathbb{R}^{N}, \mathbb{R}^{l}\right)\right), q \in W^{1,2}\left(0, T, L_{\text {loc }}^{2}\left(\mathbb{R}^{N}\right)\right)$, and that for all bounded Borel sets $B$ of $\mathbb{R}^{N}$, the real valued functions below belong to $L_{\mathbf{P}}^{1}(\Sigma)$ :

$$
\begin{aligned}
& \omega \mapsto\|r(\omega, t, \cdot)\|_{L^{2}\left(B, \mathbb{R}^{l}\right)}^{2} \text { for all } t \in[0, T], \quad \omega \mapsto\|q(\omega, t, \cdot)\|_{L^{2}(B)}^{2} \text { for all } t \in[0, T], \\
& \omega \mapsto \int_{0}^{T}\left\|\frac{d r}{d t}(\omega, \tau, \cdot)\right\|_{L^{2}\left(B, \mathbb{R}^{l}\right)}^{2} d \tau, \quad \omega \mapsto \int_{0}^{T}\left\|\frac{d q}{d t}(\omega, \tau, \cdot)\right\|_{L^{2}(B)}^{2} d \tau .
\end{aligned}
$$


Furthermore $r$ and $q$, satisfy the covariance property with respect to the dynamical system $\left(\Sigma, \mathcal{A}, \mathbf{P},\left(T_{z}\right)_{z \in \mathbb{Z}^{N}}\right)$ : for all $z \in \mathbb{Z}^{N}$, all $t \in[0,+\infty)$, a.e. $x \in \mathbb{R}^{N}$ and P-a.s. $\omega \in \Sigma$,

$$
\begin{aligned}
& r(\omega, t, x+z)=r\left(T_{z} \omega, t, x\right), \\
& q(\omega, t, x+z)=q\left(T_{z} \omega, t, x\right) .
\end{aligned}
$$

Set $f_{\varepsilon}\left(\omega, t, x, \zeta, \zeta^{\prime}\right):=f\left(\omega, t, \frac{x}{\varepsilon}, \zeta, \zeta^{\prime}\right)$, we define the functional $F_{\varepsilon}$ by setting $F_{\varepsilon}(\omega, t, u, v)(x)=f\left(\omega, t, \frac{x}{\varepsilon}, u(x), v(x)\right)$. Note that in the expression of (DCP), the functions $\bar{f}, \bar{y}$, and $\bar{\rho}$ may depend on $\omega$ (we sometimes omit it to shorten the notation), and that $F_{\varepsilon}(\omega, \cdot, \cdot, \cdot)$ is a DCP-structured reaction functional whose (DCP) condition is exactly that of $F(\omega, \cdot, \cdot, \cdot)$, i.e. with $\bar{f}, \bar{y}$, and $\bar{\rho}$. Since $\bar{y}$ does not depend on $\varepsilon$, condition (4.6) is automatically satisfied. Condition (4.3) holds for P-a.s. $\omega \in \Sigma$. Indeed, according to 2, Lemma 5.1], the following estimates hold for $\mathbf{P}$-a.s. $\omega \in \Sigma$ :

$$
\begin{aligned}
& \limsup _{\varepsilon \rightarrow 0} \int_{0}^{T}\left\|\frac{d r}{d t}\left(\omega, t, \frac{\dot{x}}{\varepsilon}\right)\right\|_{L^{2}\left(\Omega, \mathbb{R}^{l}\right)} d t \leq\left[T \mathcal{L}_{N}(\Omega) \mathbf{E}^{\mathcal{I}} \int_{0}^{T}\left\|\frac{d r}{d \tau}(\omega, \tau, \cdot)\right\|_{L^{2}\left(Y, \mathbb{R}^{l}\right)}^{2} d \tau\right]^{\frac{1}{2}}, \\
& \limsup _{\varepsilon \rightarrow 0} \int_{0}^{T}\left\|\frac{d q}{d t}\left(\omega, t, \frac{\dot{x}}{\varepsilon}\right)\right\|_{L^{2}(\Omega)} d t \leq\left[T \mathcal{L}_{N}(\Omega) \mathbf{E}^{\mathcal{I}} \int_{0}^{T}\left\|\frac{d q}{d t}(\omega, \tau, \cdot)\right\|_{L^{2}(Y)}^{2} d \tau\right]^{\frac{1}{2}} .
\end{aligned}
$$

The sequence of random history function, is defined by considering a sequence $\left(\eta_{\varepsilon}\right)_{n \in \mathbb{N}}$ of measurable maps $\omega \mapsto \eta_{\varepsilon}(\omega)$ from $\Sigma$ into $C_{c}\left((-\infty, 0], L^{2}(\Omega)\right)$ satisfying $\eta_{\varepsilon}(\omega)(0) \in H^{1}(\Omega), 0 \leq \eta_{\varepsilon}(\omega) \leq \bar{\rho}(\omega)$, and

$$
\sup _{\varepsilon>0} \int_{-\infty}^{0}\left\|\frac{d \eta_{\varepsilon}(\omega)}{d t}(t, \cdot)\right\|_{L^{2}(\Omega)} d t<+\infty .
$$

for $\mathbf{P}$-a.s. $\omega \in \Sigma$. We sometimes write $\eta_{\varepsilon}(\omega, \cdot)$ for $\eta_{\varepsilon}(\omega)$.

Finally, we assume that condition 4.7 is satisfied, more precisely, for $\mathbf{P}$-a.s. $\omega \in \Sigma$,

$$
0 \leq \phi \leq a_{0} \bar{\rho}(\omega) \text { on } \partial \Omega .
$$

We restrict the asymptotic analysis to sequences $\left(\mathbf{m}_{t}^{\varepsilon}\right)_{t \geq 0, \varepsilon}$ of random vector measures i.e., measurable maps $\omega \mapsto \mathbf{m}_{t}^{\varepsilon}(\omega)$ from $\Sigma$ into $\mathbf{M}_{1}^{+}\left(\mathbb{R}, L^{\infty}(\Omega)\right)$, satisfying one of the two structures described below.

5.2.1. First structure. Let $\tau: \Sigma \rightarrow[0,+\infty)^{\mathbb{R}^{N}}$ be a measurable map such that for $\mathbf{P}$-a.s $\omega \in \Sigma$, $\# \tau(\omega)\left(\mathbb{R}^{N}\right)$ is a constant denoted by $\# \tau$ (recall that $\#(E)$ denotes the cardinal of the set $E$ ). We assume that $\tau$ satisfies the covariance property $\tau(\omega)(x+z)=\tau\left(T_{z} \omega\right)(x)$ for all $z \in \mathbb{Z}^{N}$, a.e. $x \in \mathbb{R}^{N}$, and for $\mathbf{P}$ a.e. $\omega \in \Sigma$. According to Example 2.2, for each $\omega \in \Sigma$, and $t \geq 0$, we consider the vector measure $\mathbf{m}_{t}^{\varepsilon}(\omega) \in \mathbf{M}_{1}^{+}\left(\mathbb{R}, L^{\infty}(\Omega)\right)$ defined by

$$
\mathbf{m}_{t}^{\varepsilon}(\omega)=\frac{1}{\# \tau} \delta_{t-\tau(\omega, \dot{\bar{\varepsilon}})} .
$$

For each $\omega \in \Sigma,\left(\mathbf{m}_{t}^{\varepsilon}(\omega)\right)_{t \geq 0}$ is clearly a family of vector measures in $\mathbf{M}_{1}^{+}\left(\mathbb{R}, L^{\infty}(\Omega)\right)$ satisfying $\left(\mathrm{M}_{1}\right)$, $\left(\mathrm{M}_{2}\right)$. and $\left(\mathrm{M}_{3}\right)$. For all $v \in C_{c}\left((-\infty, T], L^{2}(\Omega)\right)$ we set

$$
\mathcal{T}_{\varepsilon}(\omega) v(t)=\int_{-\infty}^{t} v(s) d \mathbf{m}_{t}^{\varepsilon}(\omega)(s)=\frac{1}{\# \tau} v\left(t-\tau\left(\omega, \frac{\cdot}{\varepsilon}\right)\right)
$$

i.e., for almost all $x \in \Omega$,

$$
\mathcal{T}_{e}(\omega) v(t)(x)=\frac{1}{\# \tau} v\left(t-\tau\left(\omega, \frac{x}{\varepsilon}\right)\right)(x),
$$

which defines a Time delays-operator $\mathcal{T}_{\varepsilon}(\omega): C_{c}\left((-\infty, T], L^{2}(\Omega)\right) \rightarrow L^{2}\left(0, T, L^{2}(\Omega)\right)$ associated with $\left(\mathbf{m}_{t}^{\varepsilon}(\omega)\right)_{t \geq 0}$. Condition 4.5 is almost surely satisfied. Indeed, from Example 2.2 and Remark 2.1. for all $T>0$, the probability measure $\mu_{\varepsilon}(\omega)=\frac{1}{\#\left(\mathbb{R}^{N}\right)} \sum_{\tau_{i} \in \tau(\omega)\left(\mathbb{R}^{N}\right)} \delta_{\tau_{i}}$, which, in this case, does not depend on $\varepsilon$, satisfies for all $v \in C_{\eta_{\varepsilon}(\omega)}\left((-\infty, T], L^{2}(\Omega)\right)$

$$
\forall(s, t) \in[0, T]^{2} \quad s<t \Longrightarrow\left\|\mathcal{T}_{\varepsilon}(\omega) v(t)-\mathcal{T}_{\varepsilon}(\omega) v(s)\right\|_{L^{2}(\Omega)} \leq \int_{s}^{t}\left(\left\|\frac{d v}{d \sigma}(\cdot)\right\|_{L^{2}(\Omega)} \star \mu_{\varepsilon}(\omega)\right)(\sigma) d \sigma .
$$


5.2.2. Second structure. For each $k \in \mathbb{N}$, consider a $\mathcal{A}-\mathcal{B}\left(L^{\infty}(\Omega)\right)$ measurable map $\omega \mapsto \mathbf{d}_{k}(\omega, \cdot)$, with $\mathbf{d}_{k} \geq 0$, such that for $\mathbf{P}$-a.s $\omega \in \Sigma, \sum_{k \in \mathbb{N}}\left\|\mathbf{d}_{k}(\omega, \cdot)\right\|_{L^{\infty}(\Omega)}=1$. Set $\mathbf{d}(\omega, \cdot)=\left(\mathbf{d}_{k}(\omega, \cdot)\right)_{k \in \mathbb{N}}, \mathbf{d}_{k}^{\varepsilon}(\omega, x):=$ $\mathbf{d}_{k}\left(\omega, \frac{x}{\varepsilon}\right)$, and $\mathbf{d}^{\varepsilon}(\omega, \cdot)=\left(\mathbf{d}_{k}^{\varepsilon}(\omega, \cdot)\right)_{k \in \mathbb{N}}$. On the other hand, we consider a family $\left(\nu_{t}\right)_{t \geq 0}$ of measures $\nu_{t}=\left(\nu_{k, t}\right)_{k \in \mathbb{N}}$ where $\nu_{k, t}$ is a probability measure of $\mathcal{P}_{+}(\mathbb{R})$. We assume that the maps $t \mapsto \mathbb{1}_{(-\infty, t]} \nu_{k, t}$ are measurable and define the sequence $\left(\mathbf{m}_{t}^{\varepsilon}\right)_{t \geq 0}$ of random measures

$$
\omega \mapsto \mathbf{m}_{t}^{\varepsilon}(\omega)=\mathbf{d}^{\varepsilon}(\omega, \cdot) \cdot \nu_{t} .
$$

It is easily seen that for each $\omega \in \Sigma$ and $\varepsilon>0,\left(\mathbf{m}_{t}^{\varepsilon}\right)_{t \geq 0}$ is a family of vector measures in $\mathbf{M}_{1}^{+}\left(\mathbb{R}, L^{\infty}(\Omega)\right)$ which satisfies $\left(\mathrm{M}_{1}\right),\left(\mathrm{M}_{2}\right)$, and $\left(\mathrm{M}_{3}\right)$. The vector measures considered in Example 2.1, and Example 2.3 with the $\Gamma_{l}$-distribution delays, fulfill this particular structure. Indeed, with the notation of these examples, take $\nu_{k, t}=\delta_{t-\tau_{k}}$ for Example 2.1. $\nu_{k, t}=K(t-\tau) d \tau$ and $\mathbf{d}_{k}^{\varepsilon}(\omega, x)=\mathbf{d}\left(\omega, \frac{x}{\varepsilon}\right)$ if $k=0$, $\mathbf{d}_{k}^{\varepsilon}(\omega, x)=0$ if $k \in \mathbb{N}^{*}$ for Example 2.3

For all $\omega \in \Sigma$, and all $v$ in $C_{c}\left((-\infty, T], L^{2}(\Omega)\right)$, set

$$
\mathcal{T}_{\varepsilon}(\omega) v(t)=\int_{-\infty}^{t} v(s) d \mathbf{m}_{t}^{\varepsilon}(\omega)(s),
$$

which defines a Time delays-operator $\mathcal{T}_{\varepsilon}(\omega): C_{c}\left((-\infty, T], L^{2}(\Omega)\right) \rightarrow L^{2}\left(0, T, L^{2}(\Omega)\right)$ associated with $\left(\mathbf{m}_{t}^{\varepsilon}(\omega)\right)_{t>0}$. We assume that $\left(\mathbf{m}_{t}^{\varepsilon}\right)_{t \geq 0}$ is such that each operator $\mathcal{T}_{\varepsilon}$ satisfies almost surely 4.5). This is the case for the previous two examples, with $\mu_{\varepsilon}(\omega)=\sum_{k \in \mathbb{N}}\left\|\mathbf{d}_{k}(\omega, \cdot)\right\|_{L^{\infty}(\Omega)} \delta_{\tau_{k}}$ for Example 2.1. and $\mu_{\varepsilon}(\omega)=K(\sigma) d \sigma$ for Example 2.3 .

5.3. Almost sure convergence to the homogenized reaction-diffusion problem. Under above conditions, by combining Theorem 4.2 with the variational convergence of the sequence of random energies $\Phi_{\varepsilon}$, we intend to analyze the asymptotic behavior in $C\left(0, T, L^{2}(\Omega)\right)$ when $\varepsilon \rightarrow 0$ of $u_{\varepsilon}(\omega, \cdot)$, solution of the random delays reaction-diffusion problem:

$\left(\mathcal{P}_{\varepsilon}(\omega)\right)\left\{\begin{array}{l}\frac{d u_{\varepsilon}}{d t}(\omega, t)-\operatorname{div} D_{\xi} W\left(\omega, \frac{\dot{-}}{\varepsilon}, \nabla u_{\varepsilon}(\omega, t)\right)=F_{\varepsilon}\left(\omega, t, u_{\varepsilon}(\omega, t), \mathcal{T}_{\varepsilon}(\omega) u_{\varepsilon}(\omega, t)\right) \text { for a.e. } t \in(0, T) \\ u_{\varepsilon}(\omega, t)=\eta_{\varepsilon}(\omega, t), 0 \leq \eta_{\varepsilon}(\omega, t) \leq \bar{\rho}(\omega) \text { for all } t \in(-\infty, 0] \\ \left.\left.u_{\varepsilon}(\omega, t) \in H^{1}(\Omega), \operatorname{div} D_{\xi} W\left(\omega, \dot{\bar{\varepsilon}}, \nabla u_{\varepsilon}(\omega, t)\right) \in L^{2}(\Omega) \text { for all } t \in\right] 0, T\right], \\ a_{0} u_{\varepsilon}(\omega, t)+D_{\xi} W\left(\omega, \dot{\bar{\varepsilon}}, \nabla u_{\varepsilon}(\omega, t)\right) \cdot \mathbf{n}=\phi \text { on } \partial \Omega \text { for all } t \in[0, T] .\end{array}\right.$

We restrict our analysis to the case when $h=\left(h_{j}\right)_{j=1, \ldots, l}$ is given by $h_{j}\left(\zeta^{\prime}\right)=\zeta^{\prime \alpha_{j}}$ where $\alpha_{j} \in\{0,1\}$. This is the case of Examples 3.1 and 3.2. Recall that to shorten the notation, we write indifferently $\partial_{\xi} W^{\text {hom }}(\omega, \cdot)$ to denote the subdifferential of $W^{\text {hom }}(\omega, \cdot)$ or any of its elements.

Theorem 5.1. For each $\omega \in \Sigma$, let denote by $u_{\varepsilon}(\omega, \cdot)$ the unique solution in $C\left([0, T], L^{2}(\Omega)\right)$ of the (random) reaction-diffusion problem $\left(\mathcal{P}_{\varepsilon}(\omega)\right)$. Assume that for $\mathbf{P}$-a.s. $\omega \in \Sigma, \sup _{\varepsilon>0} \Phi_{\varepsilon}\left(\omega, \eta_{\varepsilon}(\omega, 0)\right)<+\infty$, and that there exists $\eta(\omega) \in C_{c}\left((-\infty, 0], L^{2}(\Omega)\right)$ such that $\eta_{\varepsilon}(\omega)$ converges to $\eta(\omega)$ in $C\left((-\infty, 0], L^{2}(\Omega)\right)$. Furthermore, in the case when $F_{\varepsilon}$ satisfies the first structure, assume that the support of $\eta(\omega)$ is include in a fixed compact set $[-M, 0], M>0$. Then, for $\mathbf{P}-a . s . \omega \in \Sigma, u_{\varepsilon}(\omega, \cdot)$ uniformly converges in $C\left([0, T], L^{2}(\Omega)\right)$ to $u(\omega)$ whose extension by $\eta(\omega)$ in $(-\infty, 0]$ is the unique solution of the reactiondiffusion problem

$$
\left(\mathcal{P}^{\text {hom }}(\omega)\right)\left\{\begin{array}{l}
\frac{d u}{d t}(\omega, t)-\operatorname{div}_{\xi} W^{h o m}(\omega, \nabla u(\omega, t)) \ni r_{\mathcal{T}}(\omega, u)(t) \cdot g(u(\omega, t))+\bar{q}(\omega, t) \text { for a.e. } t \in(0, T) \\
u(\omega, t)=\eta(\omega, t), 0 \leq \eta(\omega, t) \leq \bar{y}(\omega) \text { for all } t \in(-\infty, 0] \\
\left.\left.u(\omega, t) \in H^{1}(\Omega), \operatorname{div} \partial_{\xi} W^{h o m}(\omega, \nabla u(\omega, t)) \in L^{2}(\Omega) \text { for all } t \in\right] 0, T\right], \\
a_{0} u(\omega, t)+\partial_{\xi} W^{h o m}(\omega, \nabla u(\omega, t)) \cdot \mathbf{n} \ni \phi \text { on } \partial \Omega \text { for all } t \in[0, T],
\end{array}\right.
$$

where, for all $\psi \in C_{c}\left((-\infty, T], L^{2}(\Omega)\right)$ 
- First structure case:

$$
\begin{aligned}
r_{\mathcal{T}}(\omega, \psi)(t) & =\mathbf{E}^{\mathcal{I}}\left[\int_{Y} r(\cdot, t, y) \odot h\left(\frac{1}{\# \tau} \psi(t-\tau(\cdot, y))\right) d y\right](\omega) \\
\bar{q}(\omega, t) & =\mathbf{E}^{\mathcal{I}}\left[\int_{Y} q(\cdot, t, y) d y\right](\omega) .
\end{aligned}
$$

- Second structure case:

$$
\begin{aligned}
r_{\mathcal{T}}(\omega, \psi)(t) & =\mathbf{E}^{\mathcal{I}}\left[\int_{Y} r(\cdot, t, y) h\left(\mathbf{d}(\cdot, y) \cdot \int_{-\infty}^{t} \psi(s) d \mu_{t}(s)\right) d y\right](\omega) \\
\bar{q}(\omega, t) & =\mathbf{E}^{\mathcal{I}}\left[\int_{Y} q(\cdot, t, y) d y\right](\omega) .
\end{aligned}
$$

Moreover, for $\mathbf{P}$-a.e. $\omega \in \Sigma, \frac{d u_{\varepsilon}(\omega)}{d t} \rightarrow \frac{d u(\omega)}{d t}$ weakly in $L^{2}\left(0, T, L^{2}(\Omega)\right)$ and $0 \leq u(\omega) \leq \bar{y}(\omega, T)$.

When the dynamical system $\left(\Sigma, \mathcal{A}, \mathbf{P},\left(T_{z}\right)_{z \in \mathbb{Z}^{N}}\right)$ is ergodic, the historic function $\eta_{\varepsilon}$ is deterministic, i.e., $\eta_{\varepsilon}(\omega)=\eta_{\varepsilon}$ for $\mathbf{P}$-a.e. $\omega \in \Sigma$, together with $\bar{\rho}$, and $\bar{f}$, then $\left(\mathcal{P}^{\text {hom }}(\omega)\right)$ is deterministic and is given by

$$
\left(\mathcal{P}^{\text {hom }}\right)\left\{\begin{array}{l}
\frac{d u}{d t}(t)-\operatorname{div}_{\xi} W^{h o m}(\nabla u(t))=r_{\mathcal{T}}(u)(t) \cdot g(u(t))+\bar{q}(t) \text { for a.e. } t \in(0, T) \\
u=\eta \text { in }(-\infty, 0], 0 \leq \eta \leq \bar{y}(T), \\
\left.\left.u(t) \in H^{1}(\Omega), \operatorname{div}_{\xi} W^{h o m}(\nabla u(t)) \in L^{2}(\Omega) \text { for all } t \in\right] 0, T\right], \\
a_{0} u(t)+\partial_{\xi} W^{h o m}(\nabla u(t)) \cdot \mathbf{n} \ni \phi \text { on } \partial \Omega \text { for all } t \in[0, T],
\end{array}\right.
$$

where, for all $\psi \in C_{c}\left((-\infty, T], L^{2}(\Omega)\right), r_{\mathcal{T}}(\omega, \psi)$ is defined as above with the conditional expectation operator replaced by the mathematical expectation operator. Moreover, for $\mathbf{P}-a . s . \omega \in \Sigma, \frac{d u_{\varepsilon}}{d t} \rightarrow \frac{d u}{d t}$ weakly in $L^{2}\left(0, T, L^{2}(\Omega)\right)$ and $0 \leq u \leq \bar{y}(T)$.

If in addition $W$ satisfies (GD), then $\partial_{\xi} W^{\text {hom }}(\omega, \nabla u(t))$ or $\partial_{\xi} W^{\text {hom }}(\nabla u(t))$ are univalent equal to $D_{\xi} W^{\text {hom }}(\omega, \nabla u(t))$ or $D_{\xi} W^{\text {hom }}(\nabla u(t))$, and the differential inclusions are equalities.

Proof. All conditions of Theorem 4.2 are easy to check for $\mathbf{P}$-a.s. $\omega \in \Sigma$, except condition $\left(\mathrm{H}_{4}^{\prime}\right)$ (for the proof of $\left.\left(\mathrm{H}_{7}\right)\right]$ see [Proof of $\left.\left(\mathrm{H}_{6}\right)\right]$ in [2, Theorem 5.1]). Then, to conclude we have to establish that for all $\psi \in C_{c}\left((-\infty, T], L^{2}(\Omega)\right)$ satisfying $0 \leq \psi \leq \bar{y}(\omega, T)$, support $\left(\psi_{\lfloor(-\infty, 0]}\right) \subset \operatorname{support}(\eta(\omega))$, and for P-a.s. $\omega \in \Sigma$,

$$
r\left(\omega, \cdot \frac{\dot{\varepsilon}}{\varepsilon}\right) \odot h\left(\mathcal{T}_{\varepsilon}(\omega) \psi\right) \rightarrow r_{\mathcal{T}}(\omega, \psi)
$$

weakly in $L^{2}\left(0, T, L^{2}(\Omega)\right)$.

Proof for the first structure. Recall that from hypothesis, we have support $(\eta(\omega)) \subset[-M, 0]$. The proof proceeds in two steps.

Step 1. Let denote by $\mathcal{E}\left([-M, T], L^{2}(\Omega)\right)$ the space of step functions $v:[-M, T] \rightarrow L^{2}(\Omega)$. Since $L^{2}(\Omega)$ is separable, it is easy to show that there exists a countable subset $\mathcal{D} \subset \mathcal{E}\left([-M, T], L^{2}(\Omega)\right)$ which is dense in $C\left([-M, T], L^{2}(\Omega)\right)$ for the uniform convergence. In this step, we establish the claim for any $\psi \in \mathcal{D}$.

Let $\psi \in \mathcal{D}$ then for all $s \in[-M, T], \psi(s)=\sum_{i \in I} \mathbb{1}_{B_{i}}(s) \psi_{i}$ where $B_{i} \in \mathcal{B}(\mathbb{R})$, are mutually disjoint and $\psi_{i} \in L^{2}(\Omega)$. For $j \in\{1, \ldots, l\}$ and fixed $t \in[0, T]$, we have (recall that $h=\left(h_{j}\right)_{j=1, \ldots, l}$ with $\left.h_{j}\left(\zeta^{\prime}\right)=\zeta^{\prime \alpha_{j}}, \alpha_{j} \in\{0,1\}\right)$,

$$
\left(r\left(\omega, \cdot, \frac{\dot{-}}{\varepsilon}\right) \odot h\left(\mathcal{T}_{\varepsilon} \psi\right)(t)\right)_{j}=\left(\frac{1}{\# \tau}\right)^{\alpha_{j}} \sum_{i \in I} \mathbb{1}_{B_{i}}\left(t-\tau\left(\omega, \frac{\dot{q}}{\varepsilon}\right)\right) r_{j}\left(\omega, t, \frac{\dot{-}}{\varepsilon}\right) \psi_{i}^{\alpha_{j}} .
$$


As a consequence of the additive ergodic theorem, (see [4, Theorem 12.4.1] and [2, Lemma 5.2]), there exists $\Sigma_{\psi} \in \mathcal{A}$ which does not depend on $t$, with $\mathbf{P}\left(\Sigma_{\psi}\right)=1$ such that, for all $\omega \in \Sigma_{\psi}$,

$$
\left(\frac{1}{\# \tau}\right)^{\alpha_{j}} \mathbb{1}_{B_{i}}\left(t-\tau\left(\omega, \frac{\cdot}{\varepsilon}\right)\right) r_{j}\left(\omega, t, \frac{\cdot}{\varepsilon}\right)
$$

converges for the weak $\sigma\left(L^{\infty}(\Omega), L^{1}(\Omega)\right)$ topology toward

$$
\left(\frac{1}{\# \tau}\right)^{\alpha_{j}} \mathbf{E}^{\mathcal{I}}\left[\int_{Y} \mathbb{1}_{B_{i}}(t-\tau(\cdot, y)) r_{j}(\cdot, t, y) d y\right](\omega) .
$$

Since for any $\varphi \in L^{2}\left(0, T, L^{2}\left(\Omega, \mathbb{R}^{l}\right)\right)$ the function $\psi_{i}^{\alpha_{j}} \varphi_{j}(t, \cdot)$ belongs to $L^{1}(\Omega)$, we infer that for all $\omega \in \Sigma_{\psi}$, and for almost all $t \in[0, T]$

$$
\left\langle\left(r\left(\omega, \cdot, \frac{\cdot}{\varepsilon}\right) \odot h\left(\mathcal{T}_{\varepsilon} \psi\right)(t)\right)_{j}, \varphi_{j}(t, \cdot)\right\rangle
$$

converges to

$$
\int_{\Omega}\left(\frac{1}{\# \tau}\right)^{\alpha_{j}} \mathbf{E}^{\mathcal{I}}\left[\int_{Y}\left(\sum_{i \in I} \mathbb{1}_{B_{i}}(t-\tau(\cdot, y)) r_{j}(\cdot, t, y)\right) d y\right](\omega) \psi_{i}^{\alpha_{j}} \varphi_{j}(t, \cdot) d x
$$

which is nothing but

$$
\left\langle\left(\frac{1}{\# \tau}\right)^{\alpha_{j}} \mathbf{E}^{\mathcal{I}}\left[\int_{Y} r(\cdot, t, y) \odot h(\psi(t-\tau(\cdot, y))) d y\right]_{j}(\omega), \varphi_{j}(t, \cdot)\right\rangle .
$$

Therefore, for all $\varphi \in L^{2}\left(0, T, L^{2}\left(\Omega, \mathbb{R}^{l}\right)\right)$, almost every $t \in[0, T]$, and for all $\omega \in \Sigma^{\prime}=\bigcap_{\psi \in \mathcal{D}} \Sigma_{\psi}$,

$$
\left\langle r\left(\omega, t, \frac{\cdot}{\varepsilon}\right) \odot h\left(\mathcal{T}_{\varepsilon}(\omega) \psi\right), \varphi(t, \cdot)\right\rangle \rightarrow\left\langle r_{\mathcal{T}}(\omega, \psi), \varphi(t, \cdot)\right\rangle .
$$

The claim follows by applying the Lebesgue dominated convergence theorem. The domination property is indeed a straightforward consequence of $0 \leq \mathcal{T}_{\varepsilon}(\omega) \psi \leq \bar{y}(\omega, T)$ obtained from $0 \leq \psi \leq \bar{y}(\omega, T)$ and Lemma 2.1.

Step 2.(General case) Let $\psi \in C\left([-M, T], L^{2}(\Omega)\right)$. Then there exists a sequence $\left(\psi_{n}\right)_{n \in \mathbb{N}}$ of $\mathcal{D}$ such that $\psi_{n} \rightarrow \psi$ uniformly in $C\left([-M, T], L^{2}(\Omega)\right)$. From Step 1, there exists a set $\Sigma^{\prime} \in \mathcal{A}$ of full probability such that for all $\omega \in \Sigma^{\prime}$,

$$
r\left(\omega, \cdot, \frac{\dot{\varepsilon}}{\varepsilon}\right) \odot h\left(\mathcal{T}_{\varepsilon}(\omega) \psi_{n}\right) \rightarrow r_{\mathcal{T}}\left(\omega, \psi_{n}\right)
$$

weakly in $L^{2}\left(0, T, L^{2}(\Omega)\right)$ when $\varepsilon \rightarrow 0$. By using the conditional Lebesgue dominated convergence theorem, it is easily seen that for all $\omega \in \Sigma^{\prime}$,

$$
r_{\mathcal{T}}\left(\omega, \psi_{n}\right) \rightarrow r_{\mathcal{T}}(\omega, \psi)
$$

strongly in $L^{2}\left(0, T, L^{2}(\Omega)\right)$ when $n \rightarrow+\infty$. Write

$$
\begin{aligned}
& r\left(\omega, \cdot, \frac{\dot{ }}{\varepsilon}\right) \odot h\left(\mathcal{T}_{\varepsilon}(\omega) \psi\right) \\
& =\left(r\left(\omega, \cdot, \frac{\dot{\varepsilon}}{\varepsilon}\right) \odot h\left(\mathcal{T}_{\varepsilon}(\omega) \psi\right)-r\left(\omega, \cdot, \frac{\dot{\varepsilon}}{\varepsilon}\right) \odot h\left(\mathcal{T}_{\varepsilon}(\omega) \psi_{n}\right)\right)+r\left(\omega, \cdot, \frac{\dot{\varepsilon}}{\varepsilon}\right) \odot h\left(\mathcal{T}_{\varepsilon}(\omega) \psi_{n}\right) .
\end{aligned}
$$

Let denote by $L_{[0, \bar{y}(\omega, T)]}^{\prime}$ the Lipschitz constant of $h$ on $[0, \bar{y}(\omega, T)]$. The claim follows easily by letting first $\varepsilon \rightarrow 0$, then $n \rightarrow+\infty$ in $(5.3)$ thank's to (5.1), 5.2) and estimate

$$
\begin{aligned}
& \left\|r\left(\omega, t, \frac{\dot{-}}{\varepsilon}\right) \odot h\left(\mathcal{T}_{\varepsilon}(\omega) \psi(t)\right)-r\left(\omega, t, \frac{\dot{-}}{\varepsilon}\right) \odot h\left(\mathcal{T}_{\varepsilon}(\omega) \psi_{n}(t)\right)\right\|_{L^{2}\left(\Omega ; \mathbb{R}^{l}\right)} \\
& \leq\|r(\omega)\|_{L^{\infty}\left([0, T] \times \mathbb{R}^{N}, \mathbb{R}^{l}\right)}\left\|h\left(\psi\left(t-\tau\left(\omega, \frac{\cdot}{\varepsilon}\right)\right)\right)-h\left(\psi_{n}\left(t-\tau\left(\omega, \frac{\dot{v}}{\varepsilon}\right)\right)\right)\right\|_{L^{2}\left(\Omega, \mathbb{R}^{l}\right)} \\
& \leq L_{[0, \bar{y}(\omega, T)]}^{\prime}\|r(\omega)\|_{L^{\infty}\left([0, T] \times \mathbb{R}^{N}, \mathbb{R}^{l}\right)}\left\|\psi\left(t-\tau\left(\omega, \frac{\dot{-}}{\varepsilon}\right)\right)-\psi_{n}\left(t-\tau\left(\omega, \frac{\dot{⿰}}{\varepsilon}\right)\right)\right\|_{L^{2}(\Omega)} \\
& \leq L_{[0, \bar{y}(\omega, T)]}^{\prime}\|r(\omega)\|_{L^{\infty}\left([0, T] \times \mathbb{R}^{N}, \mathbb{R}^{l}\right)}\left\|\psi_{n}-\psi\right\|_{C_{c}\left((-\infty, T], L^{2}(\Omega)\right)} .
\end{aligned}
$$


Proof for the second structure. Fix $t \in[0, T]$ and let $i \in\{1, \ldots, l\}$ be such that $\alpha_{i}=1$. Then

$$
\begin{aligned}
r\left(\omega, t, \frac{\dot{ }}{\varepsilon}\right) \odot h\left(\mathcal{T}_{\varepsilon} \psi(t)\right)_{i} & =r_{i}\left(\omega, t, \frac{\dot{-}}{\varepsilon}\right) \int_{-\infty}^{t} \psi(s) d\left(\mathbf{d}\left(\omega, \frac{\dot{\cdot}}{\varepsilon}\right) \cdot \mu_{t}\right)(s) \\
& =r_{i}\left(\omega, t, \frac{\dot{-}}{\varepsilon}\right) \mathbf{d}\left(\omega, \frac{\dot{-}}{\varepsilon}\right) \cdot \int_{-\infty}^{t} \psi(s) d \mu_{t}(s) \\
& =\sum_{k \in \mathbb{N}} r_{i}\left(\omega, t, \frac{\dot{-}}{\varepsilon}\right) \mathbf{d}_{k}\left(\omega, \frac{\dot{-}}{\varepsilon}\right) \int_{-\infty}^{t} \psi(s) d \mu_{k, t}(s) .
\end{aligned}
$$

Hence, for all $\varphi \in L^{2}\left(0, T, L^{2}(\Omega)\right)$,

$$
\begin{aligned}
& \left\langle r\left(\omega, t, \frac{\dot{ }}{\varepsilon}\right) \odot h\left(\mathcal{T}_{\varepsilon} \psi(t)\right)_{i}, \varphi(t, \cdot)\right\rangle \\
& =\left\langle\sum_{k \in \mathbb{N}} r_{i}\left(\omega, t, \frac{\cdot}{\varepsilon}\right) \mathbf{d}_{k}\left(\omega, \frac{\cdot}{\varepsilon}\right) \int_{-\infty}^{t} \psi(s) d \mu_{k, t}(s), \varphi(t, \cdot)\right\rangle \\
& =\sum_{k \in \mathbb{N}}\left\langle r_{i}\left(\omega, t, \frac{\cdot}{\varepsilon}\right) \mathbf{d}_{k}\left(\omega, \frac{\cdot}{\varepsilon}\right) \int_{-\infty}^{t} \psi(s) d \mu_{k, t}(s), \varphi(t, \cdot)\right\rangle \\
& =\sum_{k \in \mathbb{N}}\left\langle r_{i}\left(\omega, t, \frac{\cdot}{\varepsilon}\right) \mathbf{d}_{k}\left(\omega, \frac{\cdot}{\varepsilon}\right), \varphi(t, \cdot) \int_{-\infty}^{t} \psi(s) d \mu_{k, t}(s)\right\rangle .
\end{aligned}
$$

We have used the domination property:

$$
\left\|r_{i}\left(\omega, t, \frac{\cdot}{\varepsilon}\right) \mathbf{d}_{k}\left(\omega, \frac{\cdot}{\varepsilon}\right) \int_{-\infty}^{t} \psi(s) d \mu_{k, t}(s)\right\| \leq \mathcal{L}_{N}(\Omega)^{\frac{1}{2}}\|r(\omega)\|_{L^{\infty}\left([0, T] \times \mathbb{R}^{N}, \mathbb{R}^{l}\right)} \bar{y}(\omega, T)\left\|\mathbf{d}_{k}(\omega, \cdot)\right\|_{L^{\infty}(\Omega)},
$$

combined with $\sum_{k \in \mathbb{N}}\left\|\mathbf{d}_{k}(\omega, \cdot)\right\|_{L^{\infty}(\Omega)}=1$ to permut the sum and the scalar product in the second equality, and the fact that $\varphi(t, \cdot) \int_{-\infty}^{t} \psi(s) d \mu_{k, t}(s)$ belongs to $L^{2}(\Omega)$ in the last equality (note that $\int_{-\infty}^{t} \psi(s) d \mu_{k, t}(s)$ takes its values in $[0, \bar{y}(\omega, T)]$. As a consequence of the additive ergodic theorem, [4, Theorem 12.4.1] and [2, Lemma 5.2], there exists $\Sigma^{\prime} \in \mathcal{A}$ which does not depend on $t$, with $\mathbf{P}\left(\Sigma^{\prime}\right)=1$ such that, for all $\omega \in \Sigma^{\prime}$,

$$
r_{i}\left(\omega, t, \frac{\dot{\varepsilon}}{\varepsilon}\right) \mathbf{d}_{k}\left(\omega, \frac{\cdot}{\varepsilon}\right) \rightarrow \mathbf{E}^{\mathcal{I}}\left[\int_{Y} r_{i}(\cdot, t, y) \odot \mathbf{d}_{k}(y) d y\right](\omega)
$$

weakly in $L^{2}(\Omega)$. Combining (5.4), (5.6), and the domination property $(5.5)$, we deduce that for all $\omega \in \Sigma^{\prime}$

$$
\left\langle r\left(\omega, t, \frac{\cdot}{\varepsilon}\right) \odot h\left(\mathcal{T}_{\varepsilon} \psi(t)\right)_{i}, \varphi(t, \cdot)\right\rangle \rightarrow\left\langle\mathbf{E}^{\mathcal{I}}\left[\int_{Y} r_{i}(\cdot, t, y) \mathbf{d}(y) d y\right](\omega) \cdot \int_{-\infty}^{t} \psi(s) d \mu_{t}(s), \varphi(t, \cdot)\right\rangle .
$$

According to the Lebesgue dominated convergence theorem, we infer that

$$
\left\{t \mapsto r\left(\omega, t, \frac{\cdot}{\varepsilon}\right) \odot h\left(\mathcal{T}_{\varepsilon} \psi(t)\right)_{i}\right\} \rightarrow\left\{t \mapsto \mathbf{E}^{\mathcal{I}}\left[\int_{Y} r_{i}(\cdot, t, y) \mathbf{d}(y) d y\right](\omega) \cdot \int_{-\infty}^{t} \psi(s) d \mu_{t}(s)\right\}
$$

weakly in $L^{2}\left(0, T, L^{2}(\Omega)\right)$. By using the same arguments with $\alpha_{i}=0$, we obtain that there exists $\Sigma^{\prime \prime} \in \mathcal{A}$ with $\mathbf{P}\left(\Sigma^{\prime \prime}\right)=1$, such that for all $\omega \in \Sigma^{\prime}$,

$$
\left\{t \mapsto r\left(\omega, t, \frac{\dot{\varepsilon}}{\varepsilon}\right) \odot h\left(\mathcal{T}_{\varepsilon} \psi(t)\right)_{i}\right\} \rightarrow\left\{t \mapsto \mathbf{E}^{\mathcal{I}}\left[\int_{Y} r_{i}(\cdot, t, y) d y\right](\omega)\right\}
$$

weakly in $L^{2}\left(0, T, L^{2}(\Omega)\right)$.

\section{Appendix A. Vector measures}

We give a brief summary on the concept of vector measure used to define the time-delays operators in Section 2 .

Definition A.1. Let $\mathbb{T}$ be a locally compact space and denote by $\mathcal{B}(\mathbb{T})$ its Borel $\sigma$-algebra. Given a Banach space $\mathcal{Y}$, we call $\mathcal{Y}$-valued Borel vector measure, any countably additive set function $\mathbf{m}: \mathcal{B}(\mathbb{T}) \rightarrow$ $\mathcal{Y}$. 
Let $X$ be another Banach space. We denote by $\mathcal{E}(\mathbb{T}, X)$ the space of step functions from $\mathbb{T}$ into $X$, i.e. the space of functions $S: \mathbb{T} \rightarrow X$ of the form $S=\sum_{i \in I} \mathbb{1}_{B_{i}} S_{i}$ where $I$ is any finite set, $B_{i} \in \mathcal{B}(\mathbb{T})$, and $S_{i} \in X$. Assume that there exists a continuous bilinear mapping $\mathbf{B}: \mathcal{Y} \times X \ni(u, v) \mapsto \mathbf{B}(u, v) \in X$, it follows that we can integrate with respect to $\mathbf{m}$, step functions $S \in \mathcal{E}(\mathbb{T}, X)$, and define an element of $X$, according to the formula

$$
\int S d \mathbf{m}:=\sum_{i \in I} \mathbf{B}\left(\mathbf{m}\left(B_{i}\right), S_{i}\right) \in X
$$

The definition of $\int S d \mathbf{m}$ depends only on $S$ and is independent of the particular way in which $S$ is written (see [12, Chapter II, 7, 1, Proposition 1]). We set

$$
\mathcal{B}(\mathbb{T}) \ni B \mapsto \mu(B)=\sup \left\{\sum_{i \in I}\left\|\mathbf{m}\left(B_{i}\right)\right\|_{\mathcal{Y}}: I \text { finite, }\left\{B_{i}\right\}_{i \in I} \subset \mathcal{B}(\mathbb{T}) \text { pairwise disjoint, } B=\cup_{i \in I} B_{i}\right\} .
$$

Definition A.2. The set function $\mu: \mathcal{B}(\mathbb{T}) \rightarrow[0,+\infty]$ is called the variation of $\mathbf{m}$ and is denoted by $\|\mathbf{m}\|$. The vector measure $\mathbf{m}$ has finite variation if $\|\mathbf{m}\|(\mathbb{T})<+\infty$.

The following proposition is an easy consequence of the definitions above.

Proposition A.1. Let $\mathbf{m}$ be a $\mathcal{Y}$-valued Borel measure with finite variation. Then $\|\mathbf{m}\|$ is a (scalar) nonnegative Borel measure and, for all $S \in \mathcal{E}(\mathbb{T}, X)$, we have

$$
\left\|\int S d \mathbf{m}\right\|_{X} \leq C_{\mathbf{B}} \sup _{t \in \mathbb{T}}\|S(t)\|_{X}\|\mathbf{m}\|(\mathbb{T})
$$

where $C_{\mathbf{B}}$ is the norm of the bilinear mapping $\mathbf{B}$.

Let denote by $\mathcal{E}^{\infty}(\mathbb{T}, X)$ the space of functions from $T$ into $X$ which are uniform limit of step functions. Then we have

Proposition A.2 (Extension of the integral). Let $\mathbf{m}$ be a $\mathcal{Y}$-valued Borel measure with finite variation. Then, the integral $\int S d \mathbf{m}$, defined for all $S \in \mathcal{E}(\mathbb{T}, X)$, can be extended in a standard way to the space $\mathcal{E}^{\infty}(\mathbb{T}, X)$, and for all $u \in \mathcal{E}^{\infty}(\mathbb{T}, X)$, we have

$$
\left\|\int u d \mathbf{m}\right\|_{X} \leq \int\|u\|_{X} d\|\mathbf{m}\| .
$$

Sketch of the proof. First recall the standard extension process. Let $u \in \mathcal{E}^{\infty}(\mathbb{T}, X), u=\lim _{n \rightarrow+\infty} S_{n}$ for the uniform norm, where $S_{n} \in \mathcal{E}(\mathbb{T}, X)$ for all $n \in \mathbb{N}$. Since $\left(S_{n}\right)_{n \in \mathbb{N}}$ is a Cauchy sequence for the uniform norm, from A.1,$\left(\int S_{n} d \mathbf{m}\right)_{n \in \mathbb{N}}$ is a Cauchy sequence in $X$, thus converges to some element $U$ in $X$. Let us show that $U$ does not depend on the choice of $\left(S_{n}\right)_{n \in \mathbb{N}}$. Let $\left(T_{n}\right)_{n \in \mathbb{N}}$ be any sequence in $\mathcal{E}(\mathbb{T}, X)$ uniformly converging to $u$. Then, from A.1

$$
\left\|\int\left(T_{n}-S_{n}\right) d \mathbf{m}\right\|_{X} \leq \sup _{t \in \mathbb{T}}\left\|\left(T_{n}-S_{n}\right)(t)\right\|_{X}\|\mathbf{m}\|(\mathbb{T}),
$$

so that $\lim _{n \rightarrow+\infty} \int T_{n} d \mathbf{m}=\lim _{n \rightarrow+\infty} \int S_{n} d \mathbf{m}=U$. Inequality A.2 is easy to establish for functions in $\mathcal{E}^{\infty}(\mathbb{T}, X)$ (see [12, Chapter II, 7, 2, Proposition 2]). For the general case, argue by density.

Proposition A.2 justifies the notation $\int u d \mathbf{m}$ for all $u \in \mathcal{E}^{\infty}(\mathbb{T}, X)$. It is well known that $\mathcal{E}^{\infty}(\mathbb{T}, X)$ contains the space $C_{c}(\mathbb{T}, X)$ of continuous functions from $\mathbb{T}$ into $X$ with compact support (see [12, Chapter III, 14, 5, Corollary 2]). Therefore, $\int u d \mathbf{m}$, which is sometimes written $\int_{\mathbb{T}} u(t) d \mathbf{m}(t)$, has a meaning for any $u \in C_{c}(\mathbb{T}, X)$, and defines an element of $X$. Note also that for $B \in \mathcal{B}(\mathbb{T})$, and $u \in C_{c}(\mathbb{T}, X), \mathbb{1}_{B} u$ is uniform limit of step functions so that $\int_{\mathbb{T}} \mathbb{1}_{B} u d \mathbf{m}$, written $\int_{B} u d \mathbf{m}$, has a meaning and defines an element of $X$. We refer the reader to 12 for a comprehensive review on vector measures.

\section{Appendix B. Notion of CP-STRUCtured ReACtion FunCtionals}

The definition and the theorem below are extracted from [2].

Definition B.1. Let denote by $\mathbb{R}^{\Omega}$ the set of all the maps from $\Omega$ into $\mathbb{R}$. A map $F:[0,+\infty) \times$ $L^{2}(\Omega) \rightarrow \mathbb{R}^{\Omega}$ is called a CP-structured reaction functional, if there exists a Borel measurable function 
$f:[0,+\infty) \times \mathbb{R}^{N} \times \mathbb{R} \rightarrow \mathbb{R}$ such that for all $t \in[0,+\infty)$ and all $v \in L^{2}(\Omega), F(t, v)(x)=f(t, x, v(x))$, which fulfills the following structure conditions:

$$
f(t, x, \zeta)=r(t, x) \cdot g(\zeta)+q(t, x)
$$

with

- $g: \mathbb{R} \rightarrow \mathbb{R}^{l}$ is a locally Lipschitz continuous function;

- for all $T>0, r$ belongs to $L^{\infty}\left([0, T] \times \mathbb{R}^{N}, \mathbb{R}^{l}\right)$;

- for all $T>0, q$ belongs to $L^{2}\left(0, T, L_{\text {loc }}^{2}\left(\mathbb{R}^{N}\right)\right)$.

Furthermore $f$ must satisfy the following condition:

(CP) there exist a pair $(\underline{f}, \bar{f})$ of functions $\underline{f}, \bar{f}:[0,+\infty) \times \mathbb{R} \rightarrow \mathbb{R}$ with $\underline{f} \leq 0 \leq \bar{f}$ and a pair $(\underline{\rho}, \bar{\rho})$ in $\mathbb{R}^{2}$ with $\underline{\rho} \leq \bar{\rho}$, such that each of the two following ordinary differential equations

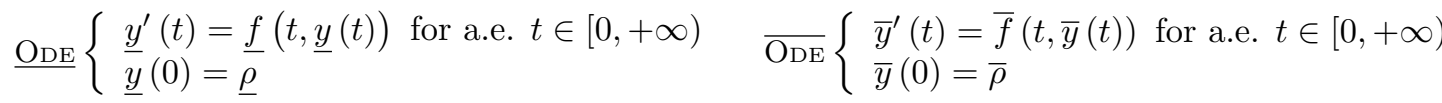

admits at least a solution denoted by $\underline{y}$ for $\underline{\mathrm{ODE}}$ and by $\bar{y}$ for $\overline{\mathrm{ODE}}$ satisfying for a.e. $(t, x) \in$ $(0,+\infty) \times \mathbb{R}$

$$
\underline{f}(t, \underline{y}(t)) \leq f(t, x, \underline{y}(t)) \quad \text { and } \quad f(t, x, \bar{y}(t)) \leq \bar{f}(t, \bar{y}(t)) .
$$

The map $F$ is referred to as a CP-structured reaction functional associated with $(r, g, q)$, and $f$ as a $C P$ structured reaction function associated with $(r, g, q)$. The map $F$ is referred to as a regular CP-structured reaction functional and $f$ as a regular CP-structured reaction function if furthermore, for all $T>0$, $r \in W^{1,1}\left(0, T, L_{\text {loc }}^{2}\left(\mathbb{R}^{N}, \mathbb{R}^{l}\right)\right)$ and $q \in W^{1,1}\left(0, T, L_{\text {loc }}^{2}\left(\mathbb{R}^{N}\right)\right)$.

Theorem B.1. Let $F$ be a CP-structured reaction functional, with $\rho, \bar{\rho}$ and $y, \bar{y}$ given by $(\mathrm{CP})$, and let $\Phi$ be a functional of the calculus of variations (3.1). Assume that $a_{0} \rho \leq h \leq a_{0} \bar{\rho}$ on $\partial \Omega$. Then for any $T>0$, the Cauchy problem

$$
(\mathcal{P})\left\{\begin{array}{l}
\frac{d u}{d t}(t)+D \Phi(u(t))=F(t, u(t)) \text { for a.e. } t \in(0, T) \\
u(0)=u_{0}, \quad \underline{\rho} \leq u_{0} \leq \bar{\rho}, \quad u_{0} \in \overline{\operatorname{dom}(D \Phi)}
\end{array}\right.
$$

admits a unique solution $u \in C\left([0, T], L^{2}(\Omega)\right)$ satisfying the following bounds in $[0, T]: \underline{y}(T) \leq \underline{y}(t) \leq$ $u(t) \leq \bar{y}(t) \leq \bar{y}(T)$. If furthermore $F$ is a regular CP-structured reaction functional, then u admits a right derivative for all $t \in[0, T[$ which satisfies $(\mathcal{P})$ for all $t \in[0, T[$.

\section{Appendix C. Estimate of the Right Derivative}

Lemma C.1. Let $X$ be a Hilbert space, $T>0, G \in W^{1,1}(0, T, X)$ and $\Phi: X \rightarrow \mathbb{R} \cup\{+\infty\}$ be a convex proper lower semicontinuous functional. Let u satisfy

$$
\left\{\begin{array}{l}
\frac{d u}{d t}(t)+\partial \Phi(u(t)) \ni G(t) \text { for a.e. } t \in(0, T), \\
u(0) \in \overline{\operatorname{dom}(\partial \Phi)} .
\end{array}\right.
$$

Then the right derivative of $u$ satisfies for all $t \in] 0, T[$ the following estimate

$$
\left\|\frac{d^{+} u}{d t}(t)\right\|_{X} \leq \frac{1}{t} \int_{0}^{t}\left\|\frac{d u}{d t}(s)\right\|_{X} d s+\int_{0}^{t}\left\|\frac{d G}{d t}(s)\right\|_{X} d s .
$$

For a proof consult [2, Lemma 3.3] 


\section{Appendix D. An alternative proof of Theorem 4.1 in the Case of a single time Delay}

We put yourself under the hypotheses of Theorem 4.1, when $\mu_{t}^{n}=\delta_{t-\tau}$, and $\tau>0$ does not depend on the space variable. We develop an alternative shorter proof of Theorem 4.1 based on the following remark: splitting $[0, T]$ into sub-intervals of size $\tau$, solving the problem

$$
\left(\mathcal{P}_{n}\right)\left\{\begin{array}{l}
\frac{d u_{n}}{d t}(t)+D \Phi_{n}\left(u_{n}(t)\right)=F_{n}\left(t, u_{n}(t), u_{n}(t-\tau)\right) \text { for a.e. } t \in(0, T) \\
u_{n}(t)=\eta_{n}(t) \text { for all } t \in[-\tau, 0]
\end{array}\right.
$$

is equivalent to solving each reaction-diffusion problem

$$
\left(\mathcal{P}_{n, i}\right)\left\{\begin{array}{l}
\frac{d u_{n}^{i}}{d t}(t)+D \Phi_{n}\left(u_{n}^{i}(t)\right)=F_{n}^{i}\left(t, u_{n}^{i}(t)\right) \text { for a.e. } t \in((i-1) \tau, i \tau) \\
u_{n}^{i}((i-1) \tau)=u_{n}^{i-1}((i-1) \tau),
\end{array}\right.
$$

for $i=1 \ldots\left[\frac{T}{\tau}\right]+1$, where $F_{n}^{i}$ is defined by $F_{n}^{i}\left(t, u_{n}^{i}(t)\right):=F_{n}\left(t, u_{n}^{i}(t), u_{n}^{i-1}(t)\right)$, and $u_{n}^{0}:=\eta_{n}$ in $[-\tau, 0]$.

Set $\bar{\rho}_{n}^{i}=\bar{y}_{n}(i \tau)$, with the initialization $\bar{\rho}_{n}^{0}=\bar{\rho}_{n}$. It is worth noting that for all $n \in \mathbb{N},\left(\bar{\rho}_{n}^{i}\right)_{i}$ is non decreasing with respect to $i$. In particular condition 4.7) yields that $0 \leq \phi_{n} \leq a_{0, n} \bar{\rho}_{n}^{i}$ on $\partial \Omega$ for $i=0 \ldots\left[\frac{T}{\tau}\right]+1$. Furthermore, since $\bar{y}_{n}$ is non decreasing, we infer that $\left[0, \bar{\rho}_{n}^{i}\right] \subset\left[0, \bar{y}_{n}(T)\right]$ for all $i=0, \ldots\left[\frac{T}{\tau}\right]+1$.

By using an inductive procedure for $i=1 \ldots\left[\frac{T}{\tau}\right]+1$, we are going to establish that $\left(\mathcal{P}_{n, i}\right)$ possesses a unique solution $u_{n}^{i}$ satisfying

$$
\begin{aligned}
& 0 \leq u_{n}^{i} \leq \bar{\rho}_{n}^{i}, \\
& \sup _{n \in \mathbb{N}} \Phi_{n}\left(u_{n}^{i}(i \tau)\right)<+\infty, \\
& u_{n}^{i} \rightarrow u^{i} \text { in } C\left([(i-1) \tau, i \tau], L^{2}(\Omega)\right), \\
& \frac{d u_{n}^{i}}{d t} \rightarrow \frac{d u^{i}}{d t} \text { in } L^{2}\left((i-1) \tau, i \tau, L^{2}(\Omega)\right),
\end{aligned}
$$

where $u^{i}$ is the unique solution of

$$
\left(\mathcal{P}_{i}\right)\left\{\begin{array}{l}
\frac{d u^{i}}{d t}(t)+\partial \Phi\left(u^{i}(t)\right) \ni F^{i}\left(t, u^{i}(t)\right) \text { for a.e. } t \in((i-1) \tau, i \tau) \\
u^{i}((i-1) \tau)=u^{i-1}((i-1) \tau)
\end{array}\right.
$$

which satisfies $0 \leq u^{i} \leq \sup _{n \in \mathbb{N}} \bar{\rho}_{n}^{i-1}$. The reaction functional $F^{i}$ will be specified at each step. We will conclude by noting that the function $u$ defined by $u=u^{i}$ on $[(i-1) \tau, i \tau]$ for $i=1 \ldots\left[\frac{T}{\tau}\right]+1$, fulfills the conditions of Theorem 4.1 .

Step $i=1$ (initialization). Since $u_{n}^{0}=\eta_{n}$ in $[-\tau, 0],\left(\mathcal{P}_{n, 1}\right)$ may be written

$$
\left(\mathcal{P}_{n, 1}\right)\left\{\begin{array}{l}
\frac{d u_{n}^{1}}{d t}(t)+D \Phi_{n}\left(u_{n}^{1}(t)\right)=F_{n}^{1}\left(t, u_{n}^{1}(t)\right) \text { for a.e. } t \in(0, \tau) \\
u_{n}^{1}(0)=\eta_{n}(0)
\end{array}\right.
$$

with $0 \leq \eta_{n}(0) \leq \bar{\rho}_{n}$ from $(\mathrm{SC} 2)$, and where $F_{n}^{1}\left(t, u_{n}^{1}(t)\right):=F_{n}\left(t, u_{n}^{1}(t), \eta_{n}(t-\tau)\right)$. Set $f_{n}^{1}(t, x, \zeta):=$ $r_{n}^{1}(t, x) \cdot g_{n}(\zeta)+q_{n}(t, x)$ where $r_{n}^{1}(t, x):=r_{n}(t, x) \odot h_{n}\left(\eta_{n}(t-\tau, x)\right)$. According to $4.2,\left(\mathrm{H}_{3}\right)$, $\left(\mathrm{H}_{5}\right)$ and $\left(\mathrm{H}_{6}\right)$ we easily deduce that $r_{n}^{1} \rightarrow r^{1}$ weakly in $L^{2}\left(0, \tau, L^{2}\left(\Omega, \mathbb{R}^{l}\right)\right)$ where $r^{1}(t, x)=r(t, x) \odot$ $h(\eta(t-\tau, x))$. Finally we check that all the conditions of [2, Theorem 4.1] are fulfilled with the time interval $(0, \tau)$ substitute for $(0, T)$. Therefore $u_{n}^{1}$ converges in $C\left([0, \tau], L^{2}(\Omega)\right)$ to the unique solution $u^{1}$ of the problem

$$
\left(\mathcal{P}_{1}\right)\left\{\begin{array}{l}
\frac{d u^{1}}{d t}(t)+\partial \Phi\left(u^{1}(t)\right) \ni F^{1}\left(t, u^{1}(t)\right) \text { for a.e. } t \in(0, \tau), \\
u^{1}(0)=\eta(0), 0 \leq \eta(0) \leq \sup _{n \in \mathbb{N}} \bar{y}_{n}(\tau)
\end{array}\right.
$$


where $F^{1}(t, v)(x)=f^{1}(t, x, v(x))$ and $f^{1}(t, x, \zeta)=r^{1}(t, x) \cdot g(\zeta)+q(t, x)$. Moreover $0 \leq u^{1} \leq \sup _{n \in \mathbb{N}} \bar{y}(\tau)$, i.e., $0 \leq u^{1} \leq \sup _{n \in \mathbb{N}} \bar{\rho}_{n}^{1}$, and $\frac{d u_{n}^{1}}{d t} \rightarrow \frac{d u^{1}}{d t}$ in $L^{2}\left(0, \tau, L^{2}(\Omega)\right)$.

It remains to establish that $\sup _{n \in \mathbb{N}} \Phi_{n}\left(u_{n}^{1}(\tau)\right)<+\infty$. As we usually do to shorten the notation, we denote by $X$ the space $L^{2}(\Omega)$. According to the proof of Theorem 4.1. Step 1, we have

$$
\begin{aligned}
& \sup _{n \in \mathbb{N}}\left\|\frac{d u_{n}^{1}}{d t}\right\| \|_{L^{2}(0, \tau, X)}<+\infty, \\
& \sup _{n \in \mathbb{N}} \int_{0}^{\tau}\left\|F_{n}^{1}\left(t, u_{n}^{1}(t)\right)\right\|_{X}^{2} d t<+\infty .
\end{aligned}
$$

Taking $\frac{d u_{n}^{1}}{d t}(t)$ as a test function, from $\left(\mathcal{P}_{n, 1}\right)$ we obtain

$$
\left\|\frac{d u_{n}^{1}}{d t}(t)\right\|_{X}^{2}+\left\langle\nabla \Phi_{n}\left(u_{n}^{1}(t)\right), \frac{d u_{n}^{1}}{d t}(t)\right\rangle=\left\langle F_{n}^{1}\left(t, u_{n}^{1}(t)\right), \frac{d u_{n}^{1}}{d t}(t)\right\rangle .
$$

Thus (see Proposition [4, Proposition 17.2.5])

$$
\frac{d}{d t} \Phi_{n}\left(u_{n}^{1}(t)\right)=\left\langle F_{n}^{1}\left(t, u_{n}^{1}(t)\right), \frac{d u_{n}^{1}}{d t}(t)\right\rangle-\left\|\frac{d u_{n}^{1}}{d t}(t)\right\|_{X}^{2}
$$

Integrating this equality over $(0, \tau)$ yields

$$
\begin{aligned}
\Phi_{n}\left(u_{n}^{1}(\tau)\right) & =\Phi_{n}\left(u_{n}^{1}(0)\right)+\int_{0}^{\tau}\left\langle F_{n}^{1}\left(t, u_{n}^{1}(t)\right), \frac{d u_{n}^{1}}{d t}(t)\right\rangle d t-\int_{0}^{\tau}\left\|\frac{d u_{n}^{1}}{d t}(t)\right\|_{X}^{2} d t \\
& =\Phi_{n}\left(\eta_{n}(0)\right)+\int_{0}^{\tau}\left\langle F_{n}^{1}\left(t, u_{n}^{1}(t)\right), \frac{d u_{n}^{1}}{d t}(t)\right\rangle d t-\int_{0}^{\tau}\left\|\frac{d u_{n}^{1}}{d t}(t)\right\|_{X}^{2} d t \\
& \leq \Phi_{n}\left(\eta_{n}(0)\right)+\left(\int_{0}^{\tau}\left\|F_{n}^{1}\left(t, u_{n}^{1}(t)\right)\right\|_{X}^{2} d t\right)^{\frac{1}{2}}\left(\int_{0}^{\tau}\left\|\frac{d u_{n}^{1}}{d t}(t)\right\|_{X}^{2} d t\right)^{\frac{1}{2}}+\int_{0}^{\tau}\left\|\frac{d u_{n}^{1}}{d t}(t)\right\|_{X}^{2} d t
\end{aligned}
$$

and $\sup _{n \in \mathbb{N}} \Phi_{n}\left(u_{n}^{1}(\tau)\right)<+\infty$ follows from hypothesis $\left(\mathrm{H}_{2}\right)$ and (D.1), (D.2).

Step $i$ starting from step $i-1, i \geq 2$. We assume that $\left(\mathcal{P}_{n, i-1}\right)$ possesses a unique solution $u_{n}^{i-1}$ satisfying

$$
\begin{aligned}
& 0 \leq u_{n}^{i-1} \leq \bar{\rho}_{n}^{i-1}, \\
& \sup _{n \in \mathbb{N}} \Phi_{n}\left(u_{n}^{i-1}((i-1) \tau)\right)<+\infty, \\
& u_{n}^{i-1} \rightarrow u^{i-1} \text { in } C\left([(i-2) \tau,(i-1) \tau], L^{2}(\Omega)\right), \\
& \frac{d u_{n}^{i-1}}{d t} \rightarrow \frac{d u^{i-1}}{d t} \text { in } L^{2}\left((i-2) \tau,(i-1) \tau, L^{2}(\Omega)\right),
\end{aligned}
$$

where $u^{i-1}$ is the unique solution of $\left(\mathcal{P}_{i-1}\right)$.

Consider the problem

$$
\left(\mathcal{P}_{n, i}\right)\left\{\begin{array}{l}
\frac{d u_{n}^{i}}{d t}(t)+D \Phi_{n}\left(u_{n}^{i}(t)\right)=F_{n}^{i}\left(t, u_{n}^{i}(t)\right) \text { for a.e. } t \in((i-1) \tau, i \tau) \\
u_{n}^{i}((i-1) \tau)=u_{n}^{i-1}((i-1) \tau)
\end{array}\right.
$$

where $F_{n}^{i}\left(t, u_{n}^{i}(t)\right):=F_{n}\left(t, u_{n}^{i}(t), u_{n}^{i-1}(t-\tau)\right)$. Set $f_{n}^{i}(t, x, \zeta):=r_{n}^{i}(t, x) \cdot g_{n}(\zeta)+q_{n}(t, x)$ where $r_{n}^{i}(t, x):=r_{n}(t, x) \odot h_{n}\left(u^{i-1}(t-\tau, x)\right)$. According to (4.2), (D.4), $\left(\mathrm{H}_{5}\right)$ and $\left(\mathrm{H}_{6}\right)$, we easily deduce that $r_{n}^{i} \rightarrow r^{i}$ weakly in $L^{2}\left((i-1) \tau, i \tau, L^{2}\left(\Omega, \mathbb{R}^{l}\right)\right)$ where $r^{i}(t, x)=r(t, x) \odot h\left(u^{2-1}(t-\tau, x)\right)$. Finally we check that all the conditions of [2, Theorem 4.1] are fulfilled where the time interval $(0, T)$ is substitute 
for $((i-1) \tau, i \tau)$. Therefore $u_{n}^{i}$ converges in $C\left([(i-1) \tau, i \tau],, L^{2}(\Omega)\right)$ to the unique solution $u^{i}$ of the problem

$$
\left(\mathcal{P}_{i}\right)\left\{\begin{array}{l}
\frac{d u^{i}}{d t}(t)+\partial \Phi\left(u^{i}(t)\right) \ni F^{1}\left(t, u^{i}(t)\right) \text { for a.e. } t \in((i-1) \tau, i \tau), \\
u^{i}((i-1) \tau)=u^{i-1}((i-1) \tau), \inf _{n} \underline{y}(i \tau) \leq u^{i-1}((i-1) \tau) \leq \sup _{n \in \mathbb{N}} \bar{y}(i \tau),
\end{array}\right.
$$

where $F^{i}(t, v)(x)=f^{i}(t, x, v(x))$ and $f^{i}(t, x, \zeta)=r^{i}(t, x) \cdot g(\zeta)+q(t, x)$. Moreover $0 \leq u^{i} \leq$ $\sup _{n \in \mathbb{N}} \bar{y}_{n}(i \tau)$, that is $0 \leq u^{i} \leq \sup _{n \in \mathbb{N}} \bar{\rho}_{n}^{i}$. The proof of $\sup _{n} \Phi_{n}\left(u_{n}^{i}(\tau)\right)<+\infty$ is similar to that of step $\mathrm{i}=1$, by using estimates

together with (D.3).

$$
\begin{aligned}
& \sup _{n \in \mathbb{N}}\left\|\frac{d u_{n}^{i}}{d t}\right\|_{L^{2}((i-1) \tau, i \tau, X)}<+\infty, \\
& \sup _{n \in \mathbb{N}} \int_{(i-1) \tau}^{i \tau}\left\|F_{n}^{i}\left(t, u_{n}^{i}(t)\right)\right\|_{X}^{2} d t<+\infty,
\end{aligned}
$$

Last step. By finite induction we construct a function $u$ defined by $u=u^{i}$ on $[(i-1) \tau, i \tau]$ for $i=1 \ldots\left[\frac{T}{\tau}\right]+1$. By construction $u$ fulfills all the conditions of the limit solution in Theorem 4.1. This completes the proof.

\section{Appendix E. Appplication of Theorem 5.1 to some examples}

E.1. Homogenization of vector disease models. In the context of vector disease models illustrated in Example 3.1. we consider the delays reaction-diffusion problem modeling the evolution of the density $u_{\varepsilon}(\omega, \cdot)$ of some infected population during a time $T>0$, in a $C^{1}$-regular domain $\Omega$. We assume that $\Omega$ is included in a $\varepsilon$-random checkerboard-like environment, or in an environment made up of spherical heterogeneities of $\operatorname{size} \varepsilon$, independently randomly distributed with a given frequency following a Poisson point process (see [2, Appendix B] for a complete description). Recall that the dynamical system $\left(\Sigma, \mathcal{A}, \mathbf{P},\left(T_{z}\right)_{z \in \mathbb{Z}^{N}}\right)$ modeling these two situations is ergodic. We assume that each two functions $a(\omega, \cdot, \cdot)$ and $b(\omega, \cdot, \cdot)$ belong to $W^{1,2}\left(0, T, L_{l o c}^{2}\left(\mathbb{R}^{N}\right)\right) \cap L^{\infty}\left([0, T] \times \mathbb{R}^{N}\right)$. When no infected population is located at the boundary, and the history function $\eta_{\varepsilon}$ is deterministic and satisfies $0 \leq \eta_{\varepsilon} \leq \bar{\rho}$ for some $\bar{\rho} \geq 1$, the density $u_{\varepsilon}(\omega, \cdot)$ is the unique solution of

$$
\left\{\begin{array}{l}
\begin{array}{r}
\frac{d u_{\varepsilon}}{d t}(\omega, t)-\operatorname{div} D_{\xi} W\left(\omega, \frac{x}{\varepsilon}, \nabla u_{\varepsilon}(\omega, t)\right)=a\left(\omega, t, \frac{\dot{ }}{\varepsilon}\right)\left(1-u_{\varepsilon}(\omega, t)\right) \int_{-\infty}^{t} u_{\varepsilon}(\omega, s) d \mathbf{m}_{t}^{\varepsilon}(s) \\
-b\left(\omega, t, \frac{\dot{\alpha}}{\varepsilon}\right) u_{\varepsilon}(\omega, t) \quad \text { for a.e. } t \in(0, T),
\end{array} \\
u_{\varepsilon}(\omega, t)=\eta_{\varepsilon}(t), \quad 0 \leq \eta_{\varepsilon}(t) \leq \bar{\rho} \text { for all } t \in(-\infty, 0], \\
\left.\left.u_{\varepsilon}(\omega, t) \in H^{1}(\Omega), \operatorname{div} D_{\xi} W\left(\omega, \dot{\bar{\varepsilon}}, \nabla u_{\varepsilon}(\omega, t)\right) \in L^{2}(\Omega) \text { for all } t \in\right] 0, T\right], \\
u_{\varepsilon}(\omega, t)=0 \text { on } \partial \Omega, \text { for all } t \in[0, T],
\end{array}\right.
$$

and satisfies $0 \leq u_{\varepsilon}(\omega) \leq \bar{\rho}$. We are in the context of Theorem 5.1, with $l=2$ and

$$
r(\omega, t, x)=(a(\omega, t, x),-b(\omega, t, x)), h\left(\zeta^{\prime}\right)=\left(\zeta^{\prime}, 1\right), g(\zeta)=(1-\zeta, \zeta) .
$$

In all examples below, to shorten the notation, we assume that $W^{ \pm}$satisfy ?? so that $W^{\text {hom }}$ is Gâteaux differentiable.

a) The case of random single delay which depends on the space variable. Concerning the distributed delays, we first consider the family of random measures associated with the random single delay of the first structure described in Section 5.2.1. These measures models a disease whose incubation period varies according to the spatial heterogeneities: we have $\mathbf{m}_{t}^{\varepsilon}(\omega)=\frac{1}{\# \tau} \delta_{t-\tau(\omega, \dot{\dot{\varepsilon}})}$, and the reaction functional is given by

$$
a\left(\omega, t, \frac{\dot{ }}{\varepsilon}\right)\left(1-u_{\varepsilon}(\omega, t)\right) \frac{1}{\# \tau} u_{\varepsilon}\left(\omega, t-\tau\left(\omega, \frac{\dot{ }}{\varepsilon}\right)\right)-b\left(\omega, t, \frac{\dot{c}}{\varepsilon}\right) u_{\varepsilon}(\omega, t) .
$$


Then, assuming that for $\mathbf{P}$ a.s. $\omega \in \Sigma$ it hold $\sup _{\varepsilon} \Phi_{\varepsilon}\left(\eta_{\varepsilon}(0)\right)<+\infty$ and that $\eta_{\varepsilon}$ converges to $\eta$ in $C_{c}\left((-\infty, 0], L^{2}(\Omega)\right)$ whose support is included in $[-M, 0]$ for some $M>0$, according to Theorem 5.1 . we can say that when $\varepsilon$ is very small compared to the size of the domain, a deterministic model, well aware with the evolution of the infected population, is given by the homogenized problem

$$
\left(\mathcal{P}^{h o m}\right)\left\{\begin{array}{l}
\frac{d u}{d t}(t)-\operatorname{div} D_{\xi} W^{h o m}(\nabla u(t))=F^{h o m}(t, u(t)) \text { for a.e. } t \in(0, T) \\
u(t)=\eta(t), \quad 0 \leq \eta(t) \leq \bar{\rho} \text { for all } t \in(-\infty, 0] \\
0 \leq u(t) \leq \bar{\rho} \text { for all } t \in(-\infty, 0] \\
u(t) \in H^{1}(\Omega), \operatorname{div} D_{\xi} W^{\text {hom }}(\nabla u(t)) \in L^{2}(\Omega) \text { for all } t \in[0, T] \\
u(t)=0 \text { on } \partial \Omega \text { for all } t \in[0, T]
\end{array}\right.
$$

where,

$$
F^{h o m}(t, u(t))=\frac{1}{\# \tau} \mathbf{E}\left[\int_{Y} a(\cdot, t, y) u(t-\tau(\cdot, y)) d y\right](1-u(t))-\mathbf{E}\left[\int_{Y} b(\cdot, t, y) d y\right] u(t) .
$$

We see that the growth rate of the uninfected population and the time delay have been mixed and averaged. Let us look at two types of spatial environments.

The $\varepsilon$-random checkerboard-like environment. Assume that the spread of the disease occurs in a $\varepsilon$-random checkerboard-like environment modeling a mosaic of two kinds of small habitats, with two virus strains: the growth rate of the uninfected and infected population, the diffusion density, together with the time delay (incubation period), take two values at random on the lattice spanned by the cell $Y=(0,1)^{N}$ at scale 1 , say $a^{-}, a^{+}, b^{-}, b^{+}, W^{-}, W^{+}$, and $\tau^{-}, \tau^{+}$respectively, with a probability $p>0$ and $1-p$ in each cell. The $\varepsilon$-random checkerboard-like environment is then modeled by a ergodic dynamical system $\left(\Sigma, \mathcal{A}, \mathbf{P}_{p},\left(T_{z}\right)_{z \in \mathbb{Z}^{N}}\right)$ where $\left(\Sigma, \mathcal{A}, \mathbf{P}_{p}\right)$ is a Bernoulli product probability space, and $T_{z}$ the shift operator In this specific case we have

$$
F^{h o m}(t, u(t))=\frac{1}{2}\left[p a^{-} u\left(t-\tau^{-}\right)+(1-p) a^{+} u\left(t-\tau^{+}\right)\right](1-u(t))-\left[p b^{-}+(1-p) b^{+}\right] u(t) .
$$

The Poisson point process environment. We now consider the Poisson point environment with $N=2$, a little more realistic, where spherical heterogeneities with radius $\varepsilon$, are independently randomly distributed with a given frequency $\lambda$ following a Poisson point process with intensity $\lambda$. Recall that this random environment is modeled by an ergodic dynamical system $\left(\Sigma, \mathcal{A}, \mathbf{P}_{\lambda},\left(T_{z}\right)_{z \in \mathbb{R}^{2}}\right)$ where $T_{z} \omega=\omega-z$ 8 , and, for every bounded Borel set $B$, and every $k \in \mathbb{N}$,

$$
\mathbf{P}_{\lambda}(\#(\Sigma \cap B)=k)=\lambda^{k} \mathcal{L}_{2}(B)^{k} \frac{\exp \left(-\lambda \mathcal{L}_{2}(B)\right)}{k !}
$$

so that $\mathbf{E}_{\lambda}[\# \Sigma \cap B]=\lambda \mathcal{L}_{2}(B)$.

Assume that the disease consists of two virus strains, one of these being concentrated in the environment, at scale 1, made up of all the balls with radius $R$ centered at the points of the Poisson process. Then, given $R>0$, we define the random density $W$ associated with the random diffusion part, by

$$
W(\omega, x, \xi)=\left\{\begin{array}{l}
W^{-}(\xi) \text { if } x \in \bigcup_{i \in \mathbb{N}} B_{R}\left(\omega_{i}\right) \\
W^{+}(\xi) \text { otherwise. }
\end{array}\right.
$$

\footnotetext{
${ }^{8}$ Here we could take the group $\left(T_{x}\right)_{x \in \mathbb{R}^{2}}, T_{x} \omega=\omega-x$, without changing the results.
} 
Similarly we set

$$
\begin{aligned}
& a(\omega, t, x)=\left\{\begin{array}{l}
a^{-}(t) \text { if } x \in \bigcup_{i \in \mathbb{N}} B_{R}\left(\omega_{i}\right), \\
a^{+}(t) \text { otherwise. }
\end{array}\right. \\
& b(\omega, t, x)=\left\{\begin{array}{l}
b^{-}(t) \text { if } x \in \bigcup_{i \in \mathbb{N}} B_{R}\left(\omega_{i}\right), \\
b^{+}(t) \text { otherwise. }
\end{array}\right. \\
& \tau(\omega, t, x)=\left\{\begin{array}{l}
\tau^{-} \text {if } x \in \bigcup_{i \in \mathbb{N}} B_{R}\left(\omega_{i}\right), \\
\tau^{+} \text {otherwise. }
\end{array}\right.
\end{aligned}
$$

Noticing that

$$
\exists \omega \in \Sigma, \quad y \in \bigcup_{i \in \mathbb{N}} B_{R}\left(\omega_{i}\right) \Longleftrightarrow \#\left(\Sigma \cap B_{R}(y)\right) \geq 1
$$

and using Fubini's theorem, we can express

$$
F^{h o m}(t, u(t))=\frac{1}{\# \tau} \mathbf{E}_{\lambda}\left[\int_{Y} a(\cdot, t, y) u(t-\tau(\cdot, y)) d y\right](1-u(t))-\mathbf{E}_{\lambda}\left[\int_{Y} b(\cdot, t, y) d y\right] u(t) .
$$

Indeed

$$
\begin{aligned}
& \mathbf{E}_{\lambda}\left[\int_{Y} a(\cdot, t, y) u(t-\tau(\cdot, y)) d y\right](1-u(t)) \\
& =a^{+}(t) u\left(t-\tau^{+}\right)(1-u(t)) \int_{\Sigma} \int_{(0,1)^{2}} \mathbb{1}_{\left[\#\left(\Sigma \cap B_{R}(y)\right)=0\right]}(\omega, y) d y d \mathbf{P}(\omega) \\
& \quad+a^{-}(t) u\left(t-\tau^{-}\right)(1-u(t)) \int_{\Sigma} \int_{(0,1)^{2}} \mathbb{1}_{\left[\#\left(\Sigma \cap B_{R}(y)\right) \geq 1\right]}(\omega, y) d y d \mathbf{P}(\omega) \\
& =a^{+}(t) u\left(t-\tau^{+}\right)(1-u(t)) \int_{(0,1)^{2}} \int_{\Sigma} \mathbb{1}_{\left[\#\left(\Sigma \cap B_{R}(y)\right)=0\right]}(\omega, y) d \mathbf{P}(\omega) d y \\
& \quad+a^{-}(t) u\left(t-\tau^{-}\right)(1-u(t)) \int_{(0,1)^{2}} \int_{\Sigma} \mathbb{1}_{\left[\#\left(\Sigma \cap B_{R}(y)\right) \geq 1\right]}(\omega, y) d \mathbf{P}(\omega) d y \\
& =a^{+}(t) u\left(t-\tau^{+}\right)(1-u(t)) \exp \left(-\lambda \pi R^{2}\right)+a^{-}(t) u\left(t-\tau^{-}\right)(1-u(t))\left(1-\exp \left(-\lambda \pi R^{2}\right)\right) \\
& =\left[a^{-}(t) u\left(t-\tau^{-}\right)+\left(a^{+}(t) u\left(t-\tau^{+}\right)-a^{-}(t) u\left(t-\tau^{-}\right)\right) \exp \left(-\lambda \pi R^{2}\right)\right](1-u(t))
\end{aligned}
$$

and, from a similar calculation

$$
\mathbf{E}_{\lambda}\left[\int_{Y} \beta(\cdot, t, y) d y\right] u(t)=\left[b^{-}(t)+\left(b^{+}(t)-b^{-}(t)\right) \exp \left(-\lambda \pi R^{2}\right)\right] u(t) .
$$

Consequently, the homogenized reaction functional is given by

$$
\begin{aligned}
F^{h o m}(t, u(t)) & =\frac{1}{2}\left[a^{-}(t) u\left(t-\tau^{-}\right)+\left(a^{+}(t) u\left(t-\tau^{+}\right)-a^{-}(t) u\left(t-\tau^{-}\right)\right) \exp \left(-\lambda \pi R^{2}\right)\right](1-u(t)) \\
& +\frac{1}{2}\left[b^{-}(t)+\left(b^{+}(t)-\beta^{-}(t)\right) \exp \left(-\lambda \pi R^{2}\right)\right] u(t)
\end{aligned}
$$

b) The case of a random multiple delays. We consider now the case when the family of random measures corresponds to the second structure of Section 5.2 .2 derived from Example 2.1 that is $\mathbf{m}_{t}^{\varepsilon}(\omega)=$ $\sum_{i \in \mathbb{N}} \mathbf{d}_{k}(\omega, \dot{\bar{\varepsilon}}) \delta_{t-\tau_{k}}$. Then, under the same hypotheses, the limit second member becomes

$$
F^{h o m}(t, u(t))=(1-u(t)) \sum_{k \in \mathbb{N}} \mathbf{E}\left[\int_{Y} a(\cdot, t, y) \mathbf{d}_{k}(\cdot, y) d y\right] u\left(t-\tau_{k}\right)-u(t) \mathbf{E}\left[\int_{Y} b(\cdot, t, y) d y\right]
$$

which can be expressed in the two previous probabilistic cases by reproducing the calculations of a). We see that the growth rate of the uninfected population and the time delays coefficients have been mixed and averaged in such a way that the limit delays reaction-diffusion problem is a reaction diffusion equation 
with a multiple delays associated with the measure $\sum_{k \in \mathbb{N}} \mathbf{E}\left[\int_{Y} a(\cdot, y) \mathbf{d}_{k}(\cdot, y) d y\right] \delta_{t-\tau_{k}}$ incorporating the growth rate.

E.2. Homogenization of delays logistic equations with immigration. We consider the food limited model with time delays corresponding to Example 3.2 which describes the evolution of the population density of some specie at time $t$ located at $x$, during a time $T>0$, in a $C^{1}$-regular domain $\Omega$. We assume, as in the previous example, that $\Omega$ is included in a $\varepsilon$-random checkerboard-like environment, or in an environment whose spherical heterogeneities of size $\varepsilon$, are independently randomly distributed with a given frequency following a Poisson point process. When no population is located at the boundary, when the history function $\eta_{\varepsilon}$ is deterministic and satisfies $0 \leq \eta_{\varepsilon} \leq \bar{\rho}$ for some $\bar{\rho}>0$, and each two functions $a$ and $q$ belongs to $W^{1,2}\left(0, T, L_{\text {loc }}^{\infty}\left(\mathbb{R}^{N}\right)\right) \cap L^{\infty}\left([0, T] \times \mathbb{R}^{N}\right)$, with $0 \leq a(\omega, t, x) \leq \bar{a}$, and $0 \leq q(\omega, t, x) \leq \bar{q}$, then, the density $u_{\varepsilon}(\omega, \cdot)$ is the unique solution of

$$
\left\{\begin{aligned}
& \frac{d u_{\varepsilon}}{d t}(\omega, t)-\operatorname{div} D_{\xi} W\left(\omega, \frac{x}{\varepsilon}, \nabla u_{\varepsilon}(\omega, t)\right)= a\left(\omega, t, \frac{\dot{x}}{\varepsilon}\right) u_{\varepsilon}(\omega, t)\left(1-\frac{1}{K_{c a r}} \int_{-\infty}^{t} u_{\varepsilon}(\omega, s) d \mathbf{m}_{t}^{\varepsilon}(s)\right) \\
&-q(\omega, t) \text { for a.e. } t \in(0, T), \\
& u_{\varepsilon}(\omega, t)=\eta_{\varepsilon}(t), \quad 0 \leq \eta_{\varepsilon}(t) \leq \bar{\rho} \text { for all } t \in(-\infty, 0], \\
&\left.\left.u_{\varepsilon}(\omega, t) \in H^{1}(\Omega), \operatorname{div} D_{\xi} W\left(\omega, \frac{\dot{\varepsilon}}{\varepsilon}, \nabla u_{\varepsilon}(\omega, t)\right) \in L^{2}(\Omega) \text { for all } t \in\right] 0, T\right] \\
& u_{\varepsilon}(\omega, t)=0 \text { on } \partial \Omega, \text { for all } t \in[0, T],
\end{aligned}\right.
$$

and satisfies $0 \leq u_{\varepsilon}(\omega, \cdot) \leq\left(\bar{\rho}+\frac{\bar{q}}{\bar{a}}\right) \exp (\bar{a} T)-\frac{\bar{q}}{\bar{a}}=\bar{y}(T)$. Recall that $a(\omega, t, x)$ is the growth rate of the population at time $t$, located at $x$ in the $\frac{1}{\varepsilon} \Omega$ domain, and $K_{c a r}>0$ is the carrying capacity, i.e., the capacity of the environment to sustain the population. To shorten the calculations, we assume that $K_{c a r}$ is independent on the time variable.

Writing the reaction functional as $a\left(\omega, t, \frac{\dot{x}}{\varepsilon}\right) u_{\varepsilon}(\omega, t)-\frac{1}{K_{c a r}} a\left(\omega, t, \frac{\dot{x}}{\varepsilon}\right) u_{\varepsilon}(\omega, t) \int_{-\infty}^{t} u_{\varepsilon}(\omega, s) d \mathbf{m}_{t}^{\varepsilon}(s)$, we see that we are in the context of Theorem 5.1, with $l=2$ and

$$
r(\omega, t, x)=\left(a(\omega, t, x),-\frac{1}{K_{c a r}} a(\omega, t, x)\right), h\left(\zeta^{\prime}\right)=\left(1, \zeta^{\prime}\right), g(\zeta)=(\zeta, \zeta) .
$$

To model the time delays, let us take the random measure derived from Example 2.3 associated with a diffuse distributed delays $\mathbf{m}_{t}^{\varepsilon}(\omega)=\mathbf{d}(\omega, \dot{\bar{\varepsilon}}) \mathcal{K}(t-s) d s$ where $\mathbf{d}>0,\|\mathbf{d}(\omega, \cdot)\|_{L^{\infty}(\Omega)}=1$, and $K$ is the $\Gamma_{l}$-distributed delays. Note that everything happens as if in the model the carrying capacity is random, depends on the spatial variable, given by $K_{c a r, \varepsilon}(\omega, x)=\frac{K_{c a r}}{\mathbf{d}\left(\omega, \frac{x}{\varepsilon}\right)}$, with a $\Gamma_{l}$-distributed delays. Then, assuming that for $\mathbf{P}$-a.s. $\omega \in \Sigma, \sup _{\varepsilon} \Phi_{\varepsilon}\left(\eta_{\varepsilon}(0)\right)<+\infty$ and that $\eta_{\varepsilon}$ converges to $\eta$ in $C_{c}\left((-\infty, 0], L^{2}(\Omega)\right)$, according to Theorem 5.1. we can say that when $\varepsilon$ is very small compared to the size of the domain, a deterministic model, well aware with the evolution of the density population, is given by

$$
\left(\mathcal{P}^{\text {hom }}\right)\left\{\begin{array}{l}
\frac{d u}{d t}(t)-\operatorname{div} D_{\xi} W^{\text {hom }}(u(t))=F^{h o m}(t, u(t)) \text { for a.e. } t \in(0, T) \\
u(t)=\eta(t), 0 \leq \eta(t) \leq \bar{\rho}, \text { for all } t \in(-\infty, 0], \\
0 \leq u(t) \leq\left(\bar{\rho}+\frac{\bar{q}}{\bar{a}}\right) \exp (\bar{a} T)-\frac{\bar{q}}{\bar{a}} \text { for all } t \in[0, T], \\
u(t) \in H^{1}(\Omega), \operatorname{div} D_{\xi} W^{\text {hom }}(\nabla u(t)) \in L^{2}(\Omega) \text { for all } t \in[0, T], \\
u(t)=0 \text { on } \partial \Omega \text { for all } t \in[0, T],
\end{array}\right.
$$


where,

$$
\begin{aligned}
F^{h o m}(t, u(t)) & =\mathbf{E}\left[\int_{Y} a(\cdot, t, y) d y\right] u(t)-u(t) \frac{1}{K_{c a r}} \mathbf{E}\left[\int_{Y} a(\cdot, t, y) \mathbf{d}(\cdot, y) d y\right] \int_{-\infty}^{t} K(t-s) u(s) d s \\
& -\mathbf{E}\left[\int_{Y} q(\cdot, t, y) d y\right] .
\end{aligned}
$$

By reproducing the calculations of Subsection E.1 it is easy to express $F^{\text {hom }}$ for each of the two probabilistic environments. Writing $F^{\text {hom }}$ of the form

$$
\begin{aligned}
F^{h o m}(t, u(t))= & \mathbf{E}\left[\int_{Y} a(\cdot, t, y) d y\right] u(t)\left(1-\frac{\mathbf{E}\left[\int_{Y} a(\cdot, t, y) \mathbf{d}(\cdot, y) d y\right]}{K_{c a r} \mathbf{E}\left[\int_{Y} a(\cdot, t, y) d y\right]} \int_{-\infty}^{t} K(t-s) u(s) d s\right) \\
& -\mathbf{E}\left[\int_{Y} q(\cdot, t, y) d y\right],
\end{aligned}
$$

we can interpret the homogenized problem as a diffuse delays logistic equation, modeling the evolution of a density population spreading in an homogeneous environment, with a $\Gamma_{l}$-distributed delays. The growth and the immigration rates are given by $\mathbf{E}\left[\int_{Y} a(\cdot, t, y) d y\right]$ and $\mathbf{E}\left[\int_{Y} q(\cdot, t, y) d y\right]$ respectively. The effective carrying capacity, given by

$$
\bar{K}_{c a r}(t)=\frac{K_{c a r} \mathbf{E}\left[\int_{Y} a(\cdot, t, y) d y\right]}{\mathbf{E}\left[\int_{Y} a(\cdot, t, y) \mathbf{d}(\cdot, y) d y\right]},
$$

is now deterministic, constant with respect to the spatial variable, but time dependent and bigger than the carrying capacity $K_{c a r}$. Note that, if the growth rate $a(\cdot, \cdot, \cdot)$ is constant, then the carrying capacity is time-space constant and given by

$$
\bar{K}_{c a r}=K_{c a r} \frac{1}{\mathbf{E}\left[\int_{Y} \mathbf{d}(\cdot, y) d y\right]},
$$

which is not the almost sure weak limit of $K_{c a r, \varepsilon}(\omega, x)$ given by

$$
K_{c a r} \mathbf{E}\left[\int_{Y} \frac{1}{\mathbf{d}(\cdot, y)} d y\right]
$$

obtained by applying the additive ergodic theorem.

\section{REFERENCES}

[1] O. Anza Hafsa, J.-P. Mandallena, G. Michaille. Groupe de recherche Mathématiques en Cévennes. Convergence of a class of nonlinear reaction-diffusion equations and stochastic homogenization http: // mipa. unimes. fr/ preprints/ MIPA-Preprint03-2016. pdf.

[2] O. Anza Hafsa, J.-P. Mandallena, G. Michaille. Stability of a class of nonlinear reaction-diffusion equations and stochastic homogenization. https://hal.archives-ouvertes.fr/hal-01928187, 2018. To appear in Asymptotic Analysis.

[3] S.N. Armstrong, P.E. Souganidis Stochastic homogenization of HamiltonJacobi and degenerate Bellman equations in unbounded environments. J. Math. Pures Appl. (9), 97 (5) (2012), pp. 460-504

[4] Hedy Attouch, Giuseppe Buttazzo, and Gérard Michaille. Variational analysis in Sobolev and BV spaces. MOS-SIAM Series on Optimization. Society for Industrial and Applied Mathematics (SIAM), Philadelphia, PA; Mathematical Optimization Society, Philadelphia, PA, second edition, 2014. Applications to PDEs and optimization.

[5] L. Berezansky, E. Braverman. Mackey-Glass Equation with Variable Coefficients. Computers and Mathematics with Applications, 51 (2006), 1-16.

[6] H. Brézis. Opérateurs maximaux monotones et semi-groupes de contractions dans les espaces de Hilbert. NorthHolland Publishing Co., Amsterdam-London; American Elsevier Publishing Co., Inc., New York, 1973. North-Holland Mathematics Studies, No. 5. Notas de Matemática (50).

[7] P. Cardaliaguet, P.-L. Lions, P.E. Souganidis A discussion about the homogenization of moving interfaces. J. Math. Pures Appl. (9), 91 (4) (2009), pp. 339-363

[8] P. Cardaliaguet, P.E. Souganidis Homogenization and enhancement of the G-equation in random environments. Comm. Pure Appl. Math., 66 (10) (2013), pp. 1582-1628

[9] A. Ciomaga P. E. Souganidis H. V.Tran Stochastic homogenization of interfaces moving with changing sign velocity. J. Diff. Equations 258, (4), ( 2015), pp. 1025-1057 
[10] G. Dal Maso, L. Modica. Nonlinear stochastic homogenization and ergodic theory. J. Reine Angew. Math. 363, 27-43, 1986.

[11] M Duerinckx, A Gloria. Stochastic homogenization of nonconvex unbounded integral functionals with convex growth. Archive for Rational Mechanics and Analysis 221 (3), 1511-1584, 2016.

[12] N. Dinculeanu. Vector measures. International Series of Monographs in Pure and Applied Mathematics, Vol. 95. Pergamon Press, Oxford-New York-Toronto, Ont.; VEB Deutscher Verlag der Wissenschaften, Berlin, 1967.

[13] G. Friesecke. Exponentially growing solutions for a delay-diffusion equation with negative feedback. J. Differential Equations 98 (1992), 1-18.

[14] G. Friesecke. Convergence to equilibrium for delay-diffusion equations with small delay. J. Dynamic. Differential Equations 5 (1993), 89-103.

[15] W. Huang Global Dynamics for a Reaction-Diffusion Equation with Time Delay. journal of differential equations 143(1998), 293-326.

[16] E. Kosygina, F. Rezakhanlou, S.R.S. Varadhan Stochastic homogenization of HamiltonJacobiBellman equations. Comm. Pure Appl. Math., 59 (10) (2006), pp. 1489-1521

[17] S.M. Kozlov. Averaging of Random Operators. Sbornik: Mathematics, 37(2), 167-180, 1980

[18] S. Luckhaus. Global boundedness for a delay-differential equation. Trans. Amer. Math. Soc. 294 (1986), $767-774$.

[19] G.C Papanicolaou. Diffusion in Random Media. In: Keller J.B., McLaughlin D.W., Papanicolaou G.C. (eds). Surveys in Applied Mathematics. Springer, Boston, MA (1995).

[20] B. Perthame, P.E. Souganidis A homogenization approach to flashing ratchets Nonlinear Differ. Equ. Appl. 18 ( 2011) 45-58.

[21] L. Pujo-Menjouet. Blood Cell Dynamics: Half of a Century of Modelling. Math. Model. Nat. Phenom. Vol. 10, 6 (2015) 182-205.

[22] S. Ruan. Delay differential equations in single species dynamics. In Delay differential equations and applications, volume 205 of NATO Sci. Ser. II Math. Phys. Chem., pages 477-517. Springer, Dordrecht, 2006.

[23] S. Ruan. J. Wu. Reaction-Diffusion equations with infinite delay. Canad. App. Math. Quart. Vol 2, 4 (1994) $485-550$.

[24] P.E. Souganidis Stochastic homogenization of HamiltonJacobi equations and some applications. Asymptot. Anal., 20 (1) (1999), pp. 1-11

[25] J. Wu. Semigroup and integral form of partial differential equations with infinite delay. Differential Integral Equations 4 (1991) 1325-1352. 